\title{
A New Concept of Supported Amino Acid Ionic Liquids and Their Application in Metal Scavenging and Heterogeneous
}

\section{Catalysis}

Wen Chen, Yuanyuan Zhang, Liangbo Zhu, Jingbo Lan, Rugang Xie and Jingsong You*

Key Laboratory of Green Chemistry and Technology of Ministry of Education, College of Chemistry, and State Key Laboratory of Biotherapy, West China Hospital, West China Medical School, Sichuan University, 29 Wangjiang Road, Chengdu 610064, PR China Fax: 86-28-85412203; E-mail: jsyou@scu.edu.cn

\section{Supporting Information}

\section{Table of contents}

I. General procedure for the catalytic $N$-arylation of nitrogen-containing heterocycles

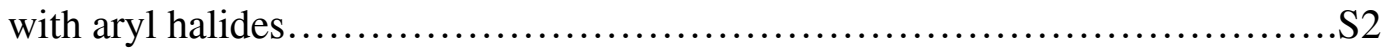

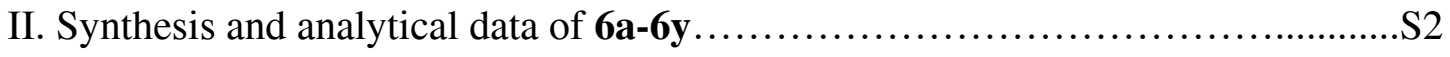

III. References................................................................

IV. Copies of ${ }^{1} \mathrm{H}$ and ${ }^{13} \mathrm{C}$ NMR spectra for compounds $\mathbf{1 a - b}$ and $\mathbf{6 a}-\mathbf{y} \ldots \ldots \ldots \ldots . . . . .514$ 


\section{General procedure for the catalytic $N$-arylation of nitrogen-containing heterocycles with aryl or heteroaryl halides}

A flame-dried Schlenk test tube with a magnetic stirring bar was charged with $\mathrm{CuI}(9.5$ mg, $0.05 \mathrm{mmol})$, 4a-2 (145 mg, $0.1 \mathrm{mmol}$ of L-proline), $\mathrm{K}_{2} \mathrm{CO}_{3}$ (165 mg, $1.2 \mathrm{mmol}$ ), nitrogen-containing heterocycle $(0.5 \mathrm{mmol})$, aryl or heteroaryl halide $(0.6 \mathrm{mmol})$ and DMSO (2 mL) under $\mathrm{N}_{2}$. A rubber septum was replaced with a glass stopper, and the system was then evacuated three times and back filled with $\mathrm{N}_{2}$. The reaction mixture was stirred for $30 \mathrm{~min}$ at room temperature, and then heated at $120^{\circ} \mathrm{C}$ for $60 \mathrm{~h}$. The resulting mixture was cooled to ambient temperature, diluted with $2-3 \mathrm{~mL}$ of ethyl acetate, filtered through a plug of silica gel, and washed with $10-20 \mathrm{~mL}$ of ethyl acetate. The filtrate was concentrated and the resulting residue was purified by column chromatography on silica gel to provide the desired product.

\section{Synthesis and analytical data of 6a-6y}

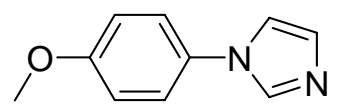

\section{1-(4-Methoxyphenyl)-1H-imidazole (6a) ${ }^{1}$}

Following the general procedure, imidazole $(0.034 \mathrm{~g}, 0.5 \mathrm{mmol})$ was coupled with 4-bromoanisole $(75 \mu \mathrm{L}, 0.6 \mathrm{mmol})$. The crude product was purified over a silica gel column using ethyl acetate/petrol ether (1/1) to give a white solid (97\% yield). ${ }^{1} \mathrm{H}$ NMR (600 MHz, $\left.\mathrm{CDCl}_{3}\right): \delta 3.86(\mathrm{~s}, 3 \mathrm{H}), 6.99(\mathrm{~d}, J=8.4 \mathrm{~Hz}, 2 \mathrm{H}), 7.20(\mathrm{bs}, 1 \mathrm{H}), 7.22(\mathrm{bs}, 1 \mathrm{H})$, $7.30(\mathrm{~d}, J=9.0 \mathrm{~Hz}, 2 \mathrm{H}), 7.79$ (bs, $1 \mathrm{H}) \mathrm{ppm} .{ }^{13} \mathrm{C} \mathrm{NMR}\left(150 \mathrm{MHz}, \mathrm{CDCl}_{3}\right): \delta 55.6,114.8$, 118.7, 123.18, 130.0, 130.7, 135.8, 158.9 ppm. GC-MS (EI, m/z): $174\left[\mathrm{M}^{+}\right]$. 


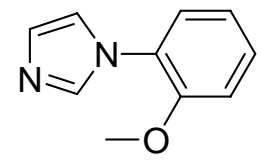

\section{1-(2-Methoxyphenyl)-1H-imidazole (6b) ${ }^{2}$}

Following the general procedure, imidazole $(0.034 \mathrm{~g}, 0.5 \mathrm{mmol})$ was coupled with 2-bromoanisole $(75 \mu \mathrm{L}, 0.6 \mathrm{mmol})$. The crude product was purified over a silica gel column using ethyl acetate/petrol ether (1/1) to give a white solid (90\% yield). ${ }^{1} \mathrm{H}$ NMR $\left(400 \mathrm{MHz}, \mathrm{CDCl}_{3}\right): \delta 3.84(\mathrm{~s}, 3 \mathrm{H}), 6.90(\mathrm{bs}, 1 \mathrm{H}), 7.02-7.07(\mathrm{~m}, 2 \mathrm{H}), 7.20(\mathrm{bs}, 1 \mathrm{H}), 7.28$ $(\mathrm{d}, J=7.6 \mathrm{~Hz}, 1 \mathrm{H}), 7.34(\mathrm{t}, J=7.6 \mathrm{~Hz}, 1 \mathrm{H}), 7.91(\mathrm{bs}, 1 \mathrm{H}) \mathrm{ppm} .{ }^{13} \mathrm{C} \mathrm{NMR}(50 \mathrm{MHz}$ $\left.\mathrm{CDCl}_{3}\right): \delta 55.6,112.2,120.2,120.8,125.3,126.3,128.4,128.8,137.6,152.4 \mathrm{ppm}$. GC-MS (EI, m/z): $174\left[\mathrm{M}^{+}\right]$.

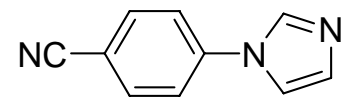

\section{4-Imidazol-1-yl-benzonitrile (6c) ${ }^{1}$}

Following the general procedure, imidazole $(0.034 \mathrm{~g}, 0.5 \mathrm{mmol})$ was coupled with 4-bromobenzonitrile $(0.108 \mathrm{~g}, 0.6 \mathrm{mmol})$ for $60 \mathrm{~h}$ at $90{ }^{\circ} \mathrm{C}$. The crude product was purified over a silica gel column using ethyl acetate/petrol ether (1/1) to give a white solid (95\% yield). ${ }^{1} \mathrm{H}$ NMR (600 MHz, $\left.\mathrm{CDCl}_{3}\right): \delta 7.27(\mathrm{~s}, 1 \mathrm{H}), 7.35$ (s, 1H), 7.54 (d, J= $8.4 \mathrm{~Hz}, 2 \mathrm{H}), 7.81(\mathrm{~d}, J=8.4 \mathrm{~Hz}, 2 \mathrm{H}), 7.99(\mathrm{~s}, 1 \mathrm{H}) \mathrm{ppm} .{ }^{13} \mathrm{C} \mathrm{NMR}\left(150 \mathrm{MHz}, \mathrm{CDCl}_{3}\right): \delta$ $110.9,117.5,117.8,121.3,131.4,134.1,135.2,140.4$ ppm. GC-MS (EI, m/z): 169 [M $\left.{ }^{+}\right]$

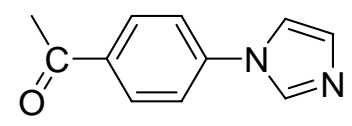




\section{1-(4-Imidazol-1-yl-phenyl)-ethanone (6d) ${ }^{3}$}

Following the general procedure, imidazole $(0.034 \mathrm{~g}, 0.5 \mathrm{mmol})$ was coupled with 4-bromoacetophenone $(0.120 \mathrm{~g}, 0.6 \mathrm{mmol})$ for $60 \mathrm{~h}$ at $90{ }^{\circ} \mathrm{C}$. The crude product was purified over a silica gel column using ethyl acetate/petrol ether (1/1) to give a white solid (92\% yield). ${ }^{1} \mathrm{H}$ NMR (600 MHz, $\left.\mathrm{CDCl}_{3}\right): \delta 2.65$ (s, 3H), 7.33 (bs, 1H), 7.45 (bs, 1H), $7.51(\mathrm{~d}, J=8.4 \mathrm{~Hz}, 2 \mathrm{H}), 8.05(\mathrm{bs}, 1 \mathrm{H}), 8.09(\mathrm{~d}, J=8.4 \mathrm{~Hz}, 2 \mathrm{H}) \mathrm{ppm} .{ }^{13} \mathrm{C}$ NMR $(75$ $\left.\mathrm{MHz}, \mathrm{CDCl}_{3}\right): \delta 26.6,117.7,120.7,130.3,131.1,135.4,135.7,140.7,196.5$ ppm. GC-MS (EI, m/z): $186\left[\mathrm{M}^{+}\right]$.

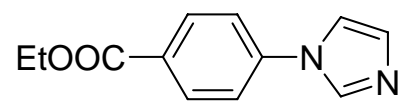

\section{4-Imidazol-1-yl-benzoic acid ethyl ester (6e) ${ }^{3}$}

Following the general procedure, imidazole $(0.034 \mathrm{~g}, 0.5 \mathrm{mmol})$ was coupled with ethyl 4-bromobenzoate $(122 \mu \mathrm{L}, 0.75 \mathrm{mmol})$ for $60 \mathrm{~h}$ at $90^{\circ} \mathrm{C}$. The crude product was purified over a silica gel column using ethyl acetate/petrol ether (1/1) to give a white solid (91\% yield). ${ }^{1} \mathrm{H}$ NMR $\left(400 \mathrm{MHz}, \mathrm{CDCl}_{3}\right): \delta 1.36(\mathrm{t}, J=7.6 \mathrm{~Hz}, 3 \mathrm{H}), 4.36(\mathrm{~m}, 2 \mathrm{H}), 7.25$ (bs, 1H), $7.36(\mathrm{bs}, 1 \mathrm{H}), 7.45-7.47(\mathrm{~m}, 2 \mathrm{H}), 7.96(\mathrm{bs}, 1 \mathrm{H}), 8.11-8.16(\mathrm{~m}, 2 \mathrm{H}) \mathrm{ppm} .{ }^{13} \mathrm{C} \mathrm{NMR}$ $\left(75 \mathrm{MHz}, \mathrm{CDCl}_{3}\right): \delta 14.3,61.3,117.9,120.1,120.5,129.4,131.1,131.5,140.6,165.5$ ppm. GC-MS (EI, m/z): $216\left[\mathrm{M}^{+}\right]$

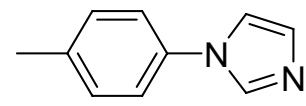

\section{1-(4-Tolyl)-1H-imidazole $(6 f)^{1}$}

Following the general procedure, imidazole $(0.034 \mathrm{~g}, 0.5 \mathrm{mmol})$ was coupled with 
4-bromotoluene $(0.103 \mathrm{~g}, 0.6 \mathrm{mmol})$. The crude product was purified over a silica gel column using ethyl acetate/petrol ether (1/1) to give a white solid (98\% yield). ${ }^{1} \mathrm{H}$ NMR (600 MHz, $\left.\mathrm{CDCl}_{3}\right): \delta 2.40(\mathrm{~s}, 3 \mathrm{H}), 7.19(\mathrm{~s}, 1 \mathrm{H}), 7.25$ (s, 1H), 7.27 (bs, 4H), $7.82(\mathrm{~s}, 1 \mathrm{H})$ ppm. ${ }^{13} \mathrm{C}$ NMR $\left(150 \mathrm{MHz}, \mathrm{CDCl}_{3}\right): \delta 20.9,118.3,121.3,130.1,130.3,134.9,135.5$, 137.4 ppm. GC-MS (EI, m/z): $158\left[\mathrm{M}^{+}\right]$.

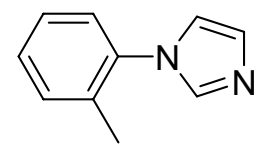

\section{1-(2-Tolyl)-1H-imidazole $(6 g)^{3}$}

Following the general procedure, imidazole $(0.034 \mathrm{~g}, 0.5 \mathrm{mmol})$ was coupled with 2-bromotoluene $(0.103 \mathrm{~g}, 0.6 \mathrm{mmol})$. The crude product was purified over a silica gel column using ethyl acetate/petrol ether (1/1) to give pale yellow oil ( $89 \%$ yield). ${ }^{1} \mathrm{H}$ NMR (400 MHz, $\left.\mathrm{CDCl}_{3}\right): \delta 2.12(\mathrm{~s}, 3 \mathrm{H}), 6.99(\mathrm{t}, J=1.2 \mathrm{~Hz}, 1 \mathrm{H}) 7.137 .31(\mathrm{~m}, 5 \mathrm{H}), 7.51$ (s, 1H) ppm. ${ }^{13} \mathrm{C}$ NMR $\left(100 \mathrm{MHz}, \mathrm{CDCl}_{3}\right): \delta 17.4,120.3,126.3,126.6,128.6,129.1$, 131.1, 133.6, 136.4, 137.3 ppm. GC-MS (EI, m/z): $158\left[\mathrm{M}^{+}\right]$

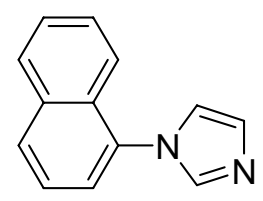

\section{1-Naphthalen-1-yl-1H-imidazole (6h)}

Following the general procedure, imidazole $(0.034 \mathrm{~g}, 0.5 \mathrm{mmol})$ was coupled with 1-bromonaphthalene ( $84 \mu \mathrm{L}, 0.6 \mathrm{mmol})$. The crude product was purified over a silica gel column using ethyl acetate/petrol ether (1/1) to give a white solid (62\% yield). ${ }^{1} \mathrm{H}$ NMR (600 MHz, $\left.\mathrm{CDCl}_{3}\right): \delta 7.26(\mathrm{bs}, 1 \mathrm{H}), 7.31(\mathrm{bs}, 1 \mathrm{H}), 7.46(\mathrm{~d}, J=7.2 \mathrm{~Hz}, 1 \mathrm{H}), 7.52-7.62(\mathrm{~m}$, 
4H), 7.78 (s, 1H), 7.95-7.97 (m, 2H) ppm. ${ }^{13} \mathrm{C}$ NMR (150 MHz, $\left.\mathrm{CDCl}_{3}\right): \delta$ 121.5, 122.1, $123.5,125.0,126.8,127.4,128.1,129.1,129.3,129.4,133.9,134.0,138.2$ ppm. GC-MS (EI, $m / z): 194\left[\mathrm{M}^{+}\right]$.

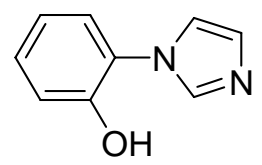

\section{2-Imidazol-1-yl-phenol (6i) ${ }^{2}$}

Following the general procedure, imidazole $(0.034 \mathrm{~g}, 0.5 \mathrm{mmol})$ was coupled with 2-bromophenol $(64 \mu \mathrm{L}, 0.6 \mathrm{mmol})$. The crude reaction mixture was dissolved in $10 \mathrm{~mL}$ of $2 \mathrm{M} \mathrm{HCl}$, and washed once with diethyl ether. The aqueous layer was brought to $\mathrm{pH}=$ 7.5 with $\mathrm{NaHCO}_{3}$, and extracted repeatedly with $\mathrm{CH}_{2} \mathrm{Cl}_{2}$. The combined organic layer was dried with anhydrous $\mathrm{MgSO}_{4}$, and concentrated under reduced pressure. The crude product was purified over a silica gel column using ethanol/ethyl acetate $(1 / 100)$ to give a white solid (74\% yield). ${ }^{1} \mathrm{H}$ NMR (400 MHz, $\left.\mathrm{CD}_{3} \mathrm{OD}\right): \delta 6.92(\mathrm{t}, J=7.6 \mathrm{~Hz}, 1 \mathrm{H}), 7.11$ (d, $J=8.4 \mathrm{~Hz}, 1 \mathrm{H}), 7.21(\mathrm{t}, J=8.0 \mathrm{~Hz}, 1 \mathrm{H}), 7.30(\mathrm{~d}, J=7.6 \mathrm{~Hz}, 1 \mathrm{H}), 7.52(\mathrm{~s}, 1 \mathrm{H}), 8.34(\mathrm{bs}$, 1H) ppm. ${ }^{13} \mathrm{C}$ NMR $\left(150 \mathrm{MHz}, \mathrm{CD}_{3} \mathrm{OD}\right.$ and $\left.\mathrm{CDCl}_{3}\right): \delta 116.7,119.3,124.7,128.1,149.9$ ppm. MS $\left(\mathrm{ESI}^{+} \mathrm{m} / \mathrm{z}\right) 161[\mathrm{M}+1]^{+}$.

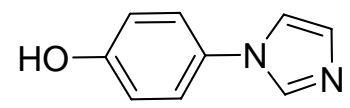

\section{4-Imidazol-1-ylphenol (6j) $)^{3}$}

Following the general procedure, imidazole $(0.034 \mathrm{~g}, 0.5 \mathrm{mmol})$ was coupled with 4-bromophenol (0.104 g, $0.6 \mathrm{mmol})$. The crude reaction mixture was dissolved in $10 \mathrm{~mL}$ of $2 \mathrm{M} \mathrm{HCl}$, and washed once with diethyl ether. The aqueous layer was brought to $\mathrm{pH}=$ 
8 with $\mathrm{Na}_{2} \mathrm{CO}_{3}$, and extracted repeatedly with $\mathrm{CH}_{2} \mathrm{Cl}_{2}$. The combined organic layer was dried with anhydrous $\mathrm{MgSO}_{4}$, and concentrated under reduced pressure. The crude product was purified over a silica gel column using ethanol/ ethyl acetate $(1 / 100)$ to give a white solid (97\% yield). ${ }^{1} \mathrm{H}$ NMR (600 MHz, $\left.\mathrm{CD}_{3} \mathrm{OD}\right): \delta 6.89(\mathrm{~d}, J=8.4 \mathrm{~Hz}, 2 \mathrm{H}), 7.11$ (bs, 1H), 7.33 (d, J = 9.0 Hz, 2H), 7.44 (bs, 1H), 7.97 (bs, 1H) ppm. ${ }^{13} \mathrm{C}$ NMR $(150 \mathrm{MHz}$, $\left.\mathrm{CD}_{3} \mathrm{OD}\right): \delta 117.3,120.3,124.2,129.7,130.7,137.1,158.5$ ppm. GC-MS (EI, m/z): 160 $\left[\mathrm{M}^{+}\right]$.

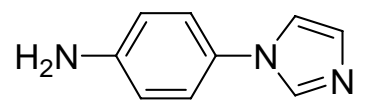

\section{4-Imidazol-1-yl-phenylamine (6k)}

Following the general procedure, imidazole $(0.034 \mathrm{~g}, 0.5 \mathrm{mmol})$ was coupled with 4-bromoaniline $(0.103 \mathrm{~g}, 0.6 \mathrm{mmol})$. The crude product was purified over a silica gel column using ethyl acetate/petrol ether (1/1) to give a white solid (86\% yield). ${ }^{1} \mathrm{H}$ NMR (600 MHz, $\left.\mathrm{CDCl}_{3}\right): \delta 3.58(\mathrm{bs}, 2 \mathrm{H}), 6.74(\mathrm{~s}, 1 \mathrm{H}), 6.75(\mathrm{~s}, 1 \mathrm{H}), 7.15-7.27(\mathrm{~m}, 4 \mathrm{H}), 7.74(\mathrm{~s}$, 1H) ppm. ${ }^{13} \mathrm{C}$ NMR $\left(150 \mathrm{MHz}, \mathrm{CDCl}_{3}\right): \delta 115.5,118.8,123.3,128.7,129.7,135.8,146.1$ ppm. GC-MS (EI, m/z): $159\left[\mathrm{M}^{+}\right]$.

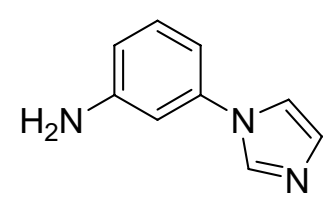

\section{3-Imidazol-1-yl-phenylamine (6l) ${ }^{3}$}

Following the general procedure, imidazole $(0.034 \mathrm{~g}, 0.5 \mathrm{mmol})$ was coupled with 3-bromoaniline $(66 \mu \mathrm{L}, 0.6 \mathrm{mmol})$. The crude product was purified over a silica gel 
column using ethyl acetate/petrol ether (1/1) to give a light yellow solid (99\% yield). ${ }^{1} \mathrm{H}$ NMR (400 MHz, $\left.\mathrm{CDCl}_{3}\right): \delta 4.00(\mathrm{bs}, 2 \mathrm{H}), 6.63-6.65(\mathrm{~m}, 2 \mathrm{H}), 6.71-6.73(\mathrm{~m}, 1 \mathrm{H})$, 7.17-7.23 (m, 3H), $7.81(\mathrm{~s}, 1 \mathrm{H}) \mathrm{ppm} .{ }^{13} \mathrm{C} \mathrm{NMR}\left(75 \mathrm{MHz}, \mathrm{CDCl}_{3}\right): \delta 107.7,111.1,113.9$, 118.3, 130.0, 130.6, 135.6, 138.3, 148.1 ppm. GC-MS (EI, $m / z): 159\left[\mathrm{M}^{+}\right]$.

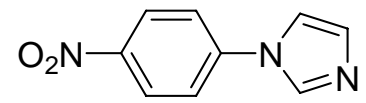

\section{1-(4-Nitrophenyl)-1H-imidazole (6m)}

Following the general procedure, imidazole $(0.034 \mathrm{~g}, 0.5 \mathrm{mmol})$ was coupled with 1-chloro-4-nitrobenzene $(0.095 \mathrm{~g}, 0.6 \mathrm{mmol})$ for $60 \mathrm{~h}$ at $90{ }^{\circ} \mathrm{C}$. The crude product was purified over a silica gel column using ethyl acetate/petrol ether (1/1) to give a pale yellow solid (97\% yield). ${ }^{1} \mathrm{H}$ NMR (600 MHz, $\left.\mathrm{CDCl}_{3}\right): \delta 7.30(\mathrm{~s}, 1 \mathrm{H}), 7.39(\mathrm{~s}, 1 \mathrm{H}), 7.59$ $(\mathrm{d}, J=9.0 \mathrm{~Hz}, 2 \mathrm{H}), 8.04(\mathrm{~s}, 1 \mathrm{H}), 8.39(\mathrm{~d}, J=9.0 \mathrm{~Hz}, 2 \mathrm{H}) \mathrm{ppm} .{ }^{13} \mathrm{C}$ NMR $(150 \mathrm{MHz}$, $\left.\mathrm{CDCl}_{3}\right): \delta 117.6,121.0,125.7,131.7,135.4,141.9,146.2 \mathrm{ppm}$. GC-MS (EI, m/z): 189 $\left[\mathrm{M}^{+}\right]$

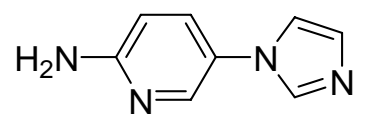

\section{5-(1H-Imidazol-1-yl)pyridin-2-amine (6n)}

Following the general procedure, imidazole $(0.034 \mathrm{~g}, 0.5 \mathrm{mmol})$ was coupled with 2-amino-5-bromopyridine $(0.104 \mathrm{~g}, 0.6 \mathrm{mmol})$. The crude product was purified over a silica gel column using ethyl acetate to give a light yellow solid (90\% yield). ${ }^{1} \mathrm{H}$ NMR (400 MHz, $\mathrm{CDCl}_{3}$ ): $\delta 4.74$ (bs, 2H), $6.57(\mathrm{~d}, J=8.8 \mathrm{~Hz}, 1 \mathrm{H}), 7.14$ (s, 1H), 7.19 (s, 1H),

$7.42(2 \mathrm{~d}, J=2.8,2.4 \mathrm{~Hz}, 1 \mathrm{H}), 7.69(\mathrm{~s}, 1 \mathrm{H}), 8.12(\mathrm{~d}, J=2.4 \mathrm{~Hz}, 1 \mathrm{H}) \mathrm{ppm} .{ }^{13} \mathrm{C}$ NMR $(75$ 
$\left.\mathrm{MHz}, \mathrm{CDCl}_{3}\right): \delta 108.7,119.5,125.7,130.3,132.5,136.2,142.1,158.2$ ppm. GC-MS (EI, $m / z): 160\left[\mathrm{M}^{+}\right]$.

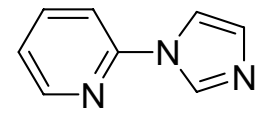

\section{2-(1H-imidazolyl)pyridine $(60)^{3}$}

Following the general procedure, imidazole $(0.034 \mathrm{~g}, 0.5 \mathrm{mmol})$ was coupled with 2-bromopyridine (59 $\mu \mathrm{L}, 0.6 \mathrm{mmol}$ ). The crude product was purified over a silica gel column using ethyl acetate/petrol ether (1/1) to give pale yellow oil (99\% yield).${ }^{1} \mathrm{H}$ NMR (300 MHz, $\left.\mathrm{CDCl}_{3}\right): \delta 7.11(\mathrm{bs}, 1 \mathrm{H}), 7.14-7.17(\mathrm{~m}, 1 \mathrm{H}), 7.26(\mathrm{~d}, J=8.2 \mathrm{~Hz}, 1 \mathrm{H})$, $7.53(\mathrm{~s}, 1 \mathrm{H}), 7.71-7.77(\mathrm{~m}, 1 \mathrm{H}), 8.28(\mathrm{bs}, 1 \mathrm{H}), 8.39(2 \mathrm{~d}, J=1.4,1.5 \mathrm{~Hz}, 1 \mathrm{H}) \mathrm{ppm} .{ }^{13} \mathrm{C}$ NMR (75 MHz, $\left.\mathrm{CDCl}_{3}\right): \delta 112.3,116.1,121.5,122.0,130.6,135.0,139.0,149.1 \mathrm{ppm}$. GC-MS (EI, m/z): $145\left[\mathrm{M}^{+}\right]$.

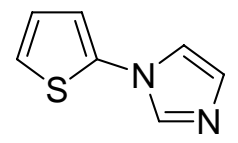

\section{1-(thiophen-2-yl)-1H-imidazole (6p) ${ }^{4}$}

Following the general procedure, imidazole $(0.034 \mathrm{~g}, 0.5 \mathrm{mmol})$ was coupled with 2-bromothiophene $(58 \mu \mathrm{L}, 0.6 \mathrm{mmol})$. The crude product was purified over a silica gel column using ethyl acetate/petrol ether $(1 / 1)$ to give pale yellow oil $(60 \%$ yield $) \cdot{ }^{1} \mathrm{H}$ NMR (400 MHz, $\left.\mathrm{CDCl}_{3}\right): \delta$ 6.94-6.97 (m, 2H), $7.10(2 \mathrm{~d}, J=1.6,2.0 \mathrm{~Hz}, 1 \mathrm{H}), 7.13(\mathrm{~s}$, $1 \mathrm{H}), 7.16(\mathrm{t}, J=1.2 \mathrm{~Hz}, 1 \mathrm{H}), 7.72(\mathrm{~s}, 1 \mathrm{H}) \mathrm{ppm} .{ }^{13} \mathrm{C} \mathrm{NMR}\left(100 \mathrm{MHz}, \mathrm{CDCl}_{3}\right): \delta 118.9$, 120.2, 121.7, 126.3, 130.2, 137.0, 139.1 ppm. GC-MS (EI, m/z): $150\left[\mathrm{M}^{+}\right]$. 
<smiles>c1cn(-c2nccs2)cn1</smiles>

\section{1-(thiazol-2-yl)-1H-imidazole (6q)}

Following the general procedure, imidazole $(0.034 \mathrm{~g}, 0.5 \mathrm{mmol})$ was coupled with 2-bromothiazole $(53 \mu \mathrm{L}, 0.6 \mathrm{mmol})$. The crude product was purified over a silica gel column using ethyl acetate/petrol ether (1/1) to give pale yellow oil (86\% yield).${ }^{1} \mathrm{H}$ NMR (400 MHz, $\left.\mathrm{CDCl}_{3}\right): \delta 7.11-7.14(\mathrm{~m}, 2 \mathrm{H}), 7.47(\mathrm{t}, J=1.2 \mathrm{~Hz}, 1 \mathrm{H}), 7.53(\mathrm{~d}, J=3.6$ $\mathrm{Hz}, 1 \mathrm{H}), 8.15(\mathrm{~s}, 1 \mathrm{H}) \mathrm{ppm} .{ }^{13} \mathrm{C} \mathrm{NMR}\left(150 \mathrm{MHz}, \mathrm{CDCl}_{3}\right): \delta 115.5,117.6,130.8,135.4$, 140.5, 157.4 ppm. GC-MS (EI, $m / z): 151\left[\mathrm{M}^{+}\right]$.<smiles>c1cnc(-n2ccnc2)nc1</smiles>

\section{2-(1H-imidazol-1-yl)pyrimidine $(6 r)^{5}$}

Following the general procedure, imidazole $(0.034 \mathrm{~g}, 0.5 \mathrm{mmol})$ was coupled with 2-chloropyrimidine $(69 \mathrm{mg}, 0.6 \mathrm{mmol})$. The crude product was purified over a silica gel column using ethyl acetate/petrol ether (1/1) to give white solid (94\% yield). ${ }^{1} \mathrm{H}$ NMR $\left(400 \mathrm{MHz}, \mathrm{CDCl}_{3}\right): \delta 7.10(\mathrm{~s}, 1 \mathrm{H}), 7.14(\mathrm{t}, J=4.8 \mathrm{~Hz}, 1 \mathrm{H}), 7.82(\mathrm{~s}, 1 \mathrm{H}), 8.55(\mathrm{~s}, 1 \mathrm{H})$,

$8.61(\mathrm{~d}, J=5.2 \mathrm{~Hz}, 2 \mathrm{H}) \mathrm{ppm} .{ }^{13} \mathrm{C} \mathrm{NMR}\left(100 \mathrm{MHz}, \mathrm{CDCl}_{3}\right): \delta 116.4,118.7,130.5,136.0$, 158.6 ppm. GC-MS (EI, m/z): $146\left[\mathrm{M}^{+}\right]$.

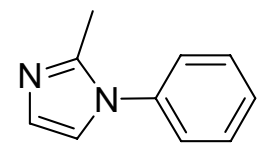

\section{1-Phenyl-2-methyl-imidazole (6s)}

Following the general procedure, 2-methyl-imidazole $(0.041 \mathrm{~g}, 0.5 \mathrm{mmol})$ was coupled 
with bromobenzene ( $63 \mu \mathrm{L}, 0.6 \mathrm{mmol})$. The crude product was purified over a silica gel column using ethyl acetate/petrol ether (1/1) to give a white solid (68\% yield). ${ }^{1} \mathrm{H}$ NMR $\left(400 \mathrm{MHz}, \mathrm{CDCl}_{3}\right): \delta 2.36(\mathrm{~s}, 3 \mathrm{H}), 7.00(\mathrm{~d}, J=10.8 \mathrm{~Hz}, 2 \mathrm{H}), 7.29-7.27(\mathrm{~m}, 2 \mathrm{H})$, 7.39-7.49 (m, 3H) ppm. ${ }^{13} \mathrm{C}$ NMR (100 MHz, $\left.\mathrm{CDCl}_{3}\right): \delta 13.7,120.6,125.4,127.7,128.1$, 129.4, 138.0, 144.5 ppm. GC-MS (EI, $m / z): 158\left[\mathrm{M}^{+}\right]$.

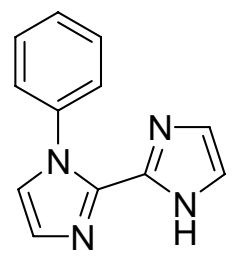

\section{2-(1H-Imidazol-2-yl)-1-phenyl-1H-imidazole (6t)}

Following the general procedure, 2-(1H-imidazol-2-yl)-1H-imidazole $(0.067 \mathrm{~g}, 0.5 \mathrm{mmol})$ was coupled with bromobenzene $(63 \mu \mathrm{L}, 0.6 \mathrm{mmol})$. The crude product was purified over a silica gel column using ethyl acetate/petrol ether (1/1) to give a pale yellow solid $(81 \%$ yield). ${ }^{1} \mathrm{H}$ NMR (600 MHz, $\left.\mathrm{CDCl}_{3}\right): \delta 7.03(\mathrm{~s}, 2 \mathrm{H}), 7.13(\mathrm{~s}, 1 \mathrm{H}), 7.21(\mathrm{~s}, 1 \mathrm{H}), 7.44-7.46$ (m, 2H), 7.48-7.50 (m, 3H) ppm. ${ }^{13} \mathrm{C}$ NMR (150 MHz, $\left.\mathrm{CDCl}_{3}\right): \delta$ 123.4, 126.3, 127.7, 128.6, 128.9, 138.1, 138.1, 139.2 ppm. GC-MS (EI, m/z): 209 [M-1] .

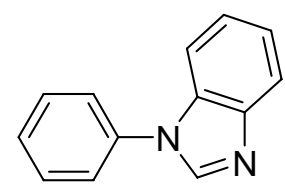

\section{1-Phenyl-1H-benzimidazole $(6 \mathrm{u})^{6}$}

Following the general procedure, benzimidazole $(0.059 \mathrm{~g}, 0.5 \mathrm{mmol})$ was coupled with iodobenzene $(67 \mu \mathrm{L}, 0.6 \mathrm{mmol})$. The crude product was purified over a silica gel column using ethyl acetate/petrol ether (1/2) to give a white solid (99\% yield). ${ }^{1} \mathrm{H}$ NMR (600 
$\left.\mathrm{MHz}, \mathrm{CDCl}_{3}\right): \delta$ 7.33-7.37 (m, 2H), $7.47(\mathrm{t}, J=7.2 \mathrm{~Hz}, 1 \mathrm{H}), 7.52-7.60(\mathrm{~m}, 5 \mathrm{H}), 7.89-7.90$

(m, 1H), 8.15 (s, $1 \mathrm{H})$ ppm. ${ }^{13} \mathrm{C}$ NMR $\left(150 \mathrm{MHz}, \mathrm{CDCl}_{3}\right): \delta 110.4,120.5,122.8,123.6$, 124.0, 128.0, 130.0, 133.6, 136.3, 142.2, 143.9 ppm. GC-MS (EI, m/z): $194\left[\mathrm{M}^{+}\right]$.

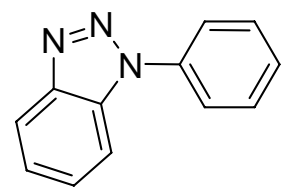

\section{1-Phenyl-1H-1,2,3-benzotriazole (6v) ${ }^{7}$}

Following the general procedure, $1 \mathrm{H}-1,2,3$-benzotriazole $(0.060 \mathrm{~g}, 0.5 \mathrm{mmol})$ was coupled with bromobenzene ( $63 \mu \mathrm{L}, 0.6 \mathrm{mmol})$. The crude product was purified over a silica gel column using ethyl acetate/petrol ether (1/4) to give pale yellow solid (93\% yield). ${ }^{1} \mathrm{H}$ NMR $\left(400 \mathrm{MHz}, \mathrm{CDCl}_{3}\right): \delta 7.42(\mathrm{t}, J=7.6 \mathrm{~Hz}, 1 \mathrm{H}), 7.49-7.57(\mathrm{~m}, 2 \mathrm{H})$, 7.60-7.63 (t, $J=8.0 \mathrm{~Hz}, 2 \mathrm{H}), 7.74-7.80(\mathrm{~m}, 3 \mathrm{H}), 8.14(\mathrm{~d}, J=8.0 \mathrm{~Hz}, 1 \mathrm{H}) \mathrm{ppm} . \mathrm{GC}-\mathrm{MS}$ (EI, $m / z): 195\left[\mathrm{M}^{+}\right]$.

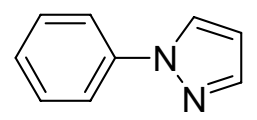

\section{1-Phenyl-1H-pyrazole $(6 \mathrm{w})^{8}$}

Following the general procedure, $1 \mathrm{H}-$ pyrazole $(0.034 \mathrm{~g}, 0.5 \mathrm{mmol})$ was coupled with bromobenzene $(63 \mu \mathrm{L}, 0.6 \mathrm{mmol})$. The crude product was purified over a silica gel column using ethyl acetate/petrol ether (1/9) to give pale yellow oil (90\% yield). ${ }^{1} \mathrm{H}$ NMR (400 MHz, $\left.\mathrm{CDCl}_{3}\right): \delta 6.43(\mathrm{t}, J=2.4 \mathrm{~Hz}, 1 \mathrm{H}), 7.24-7.28(\mathrm{~m}, 1 \mathrm{H}), 7.39-7.45(\mathrm{~m}$, $2 \mathrm{H}), 7.68-7.74(\mathrm{~m}, 3 \mathrm{H}), 7.88(\mathrm{~d}, J=2.4 \mathrm{~Hz}, 1 \mathrm{H}) \mathrm{ppm} ;{ }^{13} \mathrm{C} \mathrm{NMR}\left(75 \mathrm{MHz}, \mathrm{CDCl}_{3}\right): \delta$ 107.6, 119.2, 126.4, 126.8, 129.4, 140.2, 141.1 ppm. GC-MS (EI, m/z): $144\left[\mathrm{M}^{+}\right]$. 


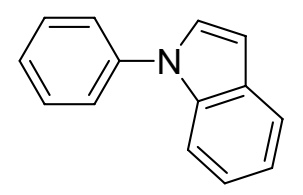

\section{1-Phenyl-1H-indole (6x) ${ }^{9}$}

Following the general procedure, $1 \mathrm{H}$-indole $(0.059 \mathrm{~g}, 0.5 \mathrm{mmol})$ was coupled with iodobenzene $(67 \mu \mathrm{L}, 0.6 \mathrm{mmol})$. The crude product was purified over a silica gel column using ethyl acetate/petrol ether (1/50) to give colorless oil (99\% yield). ${ }^{1} \mathrm{H}$ NMR (300 $\left.\mathrm{MHz}, \mathrm{CDCl}_{3}\right): \delta 6.56(\mathrm{~d}, J=3.2 \mathrm{~Hz}, 1 \mathrm{H}), 7.03-7.13(\mathrm{~m}, 2 \mathrm{H}), 7.18-7.25(\mathrm{~m}, 2 \mathrm{H})$, 7.33-7.37 (m, 4H), 7.41-7.60 (m, 2H) ppm. $\left.{ }^{13} \mathrm{C} \mathrm{NMR} \mathrm{(75} \mathrm{MHz,} \mathrm{CDCl}_{3}\right): \delta 103.7,110.6$, $120.5,121.3,122.5,124.5,126.5,128.0,129.5,130.1,136.0,140.0$ ppm. GC-MS (EI, $m / z): 193\left[\mathrm{M}^{+}\right]$.

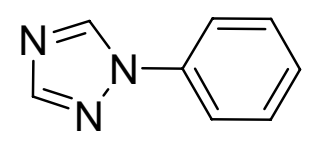

\section{1-Phenyl-1H-1,2,4-triazole (6y) ${ }^{9}$}

Following the general procedure, 1,2,4-triazole $(0.035 \mathrm{~g}, 0.5 \mathrm{mmol})$ was coupled with iodobenzene $(67 \mu \mathrm{L}, 0.6 \mathrm{mmol})$. The crude product was purified over a silica gel column using ethyl acetate/petrol ether (1/2) to give a light yellow solid (99\% yield). ${ }^{1} \mathrm{H}$ NMR $\left(300 \mathrm{MHz}, \mathrm{CDCl}_{3}\right): \delta 7.27(\mathrm{t}, J=7.0 \mathrm{~Hz}, 1 \mathrm{H}), 7.38(\mathrm{t}, J=8.0 \mathrm{~Hz}, 2 \mathrm{H}), 7.57(\mathrm{~d}, J=8.3$ $\mathrm{Hz}, 2 \mathrm{H}), 8.01(\mathrm{bs}, 1 \mathrm{H}), 8.48$ (bs, $1 \mathrm{H}) \mathrm{ppm} .{ }^{13} \mathrm{C} \mathrm{NMR}\left(75 \mathrm{MHz}, \mathrm{CDCl}_{3}\right): \delta 119.6,128.2$, 129.7, 137.0, 140.9, 152.6 ppm. GC-MS (EI, m/z): $145\left[\mathrm{M}^{+}\right]$. 


\section{References}

[1] Choudary, B. M.; Sridhar, C.; Kantam, M. L.; Venkanna, G. T.; Sreedhar, B. J. Am. Chem. Soc. 2005, 127, 9948.

[2] Collman, J. P.; Wang, Z.; Zhong, M.; Zeng, L. J. Chem. Soc., Perkin Trans. 1, 2000, 1217.

[3] Altman, R. A.; Buchwald, S. L. Org. Lett. 2006, 8, 2779.

[4] Lv, X.; Wang, Z.; Bao, W. Tetrahedron 2006, 62, 4756.

[5] Xie, Y.; Pi,S. Wang, J.; Yin, D.; Li, J. J. Org. Chem. 2006, 71, 8324.

[6] Nishiura, K.; Urawa, Y.; Sodab, S. Adv. Synth. Catal. 2004, 346, 1679.

[7] Begtrup, M.; Elguero, J.; Faure, R.; Camps, P.; Estopa, C.; Ilavsky, D.; Fruchier, A.; Marzin, C. de Mendoza, J. Magn. Reson. Chem. 1988, 26, 134.

[8] Cristau, H. J.; Cellier, P. P.; Spindler, J. F.; Taillefer, M. Eur. J. Org. Chem. 2004, 695.

[9] Cristau, H. J.; Cellier, P. P.; Spindler, J. F.; Taillefer, M. Chem.-Eur. J. 2004, 10, 5607.

\section{Copies of ${ }^{1} \mathrm{H}$ and ${ }^{13} \mathrm{C}$ NMR spectra for compounds $1 \mathrm{a}-\mathrm{b}$ and $6 \mathrm{a}-\mathrm{y}$}




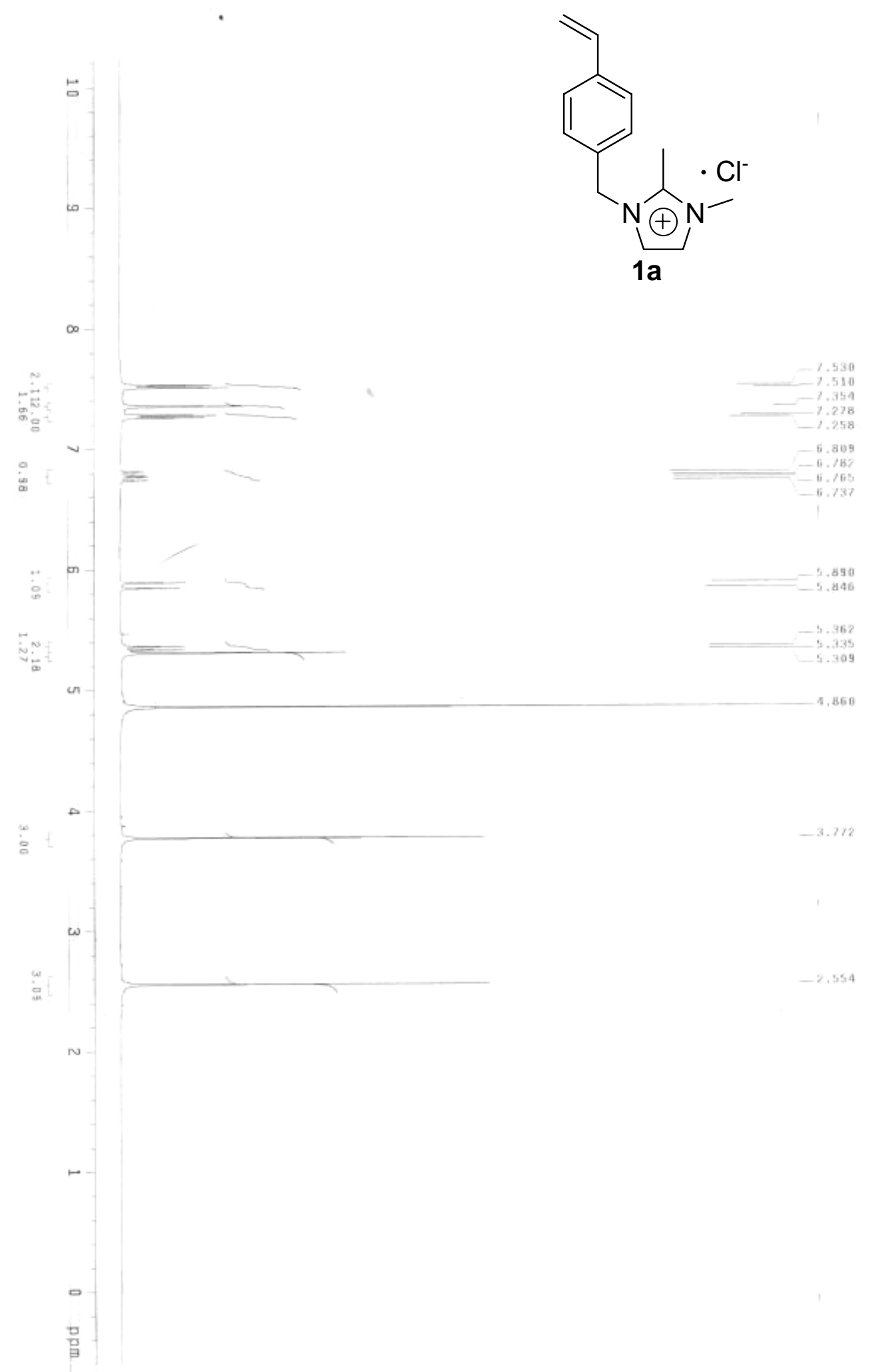




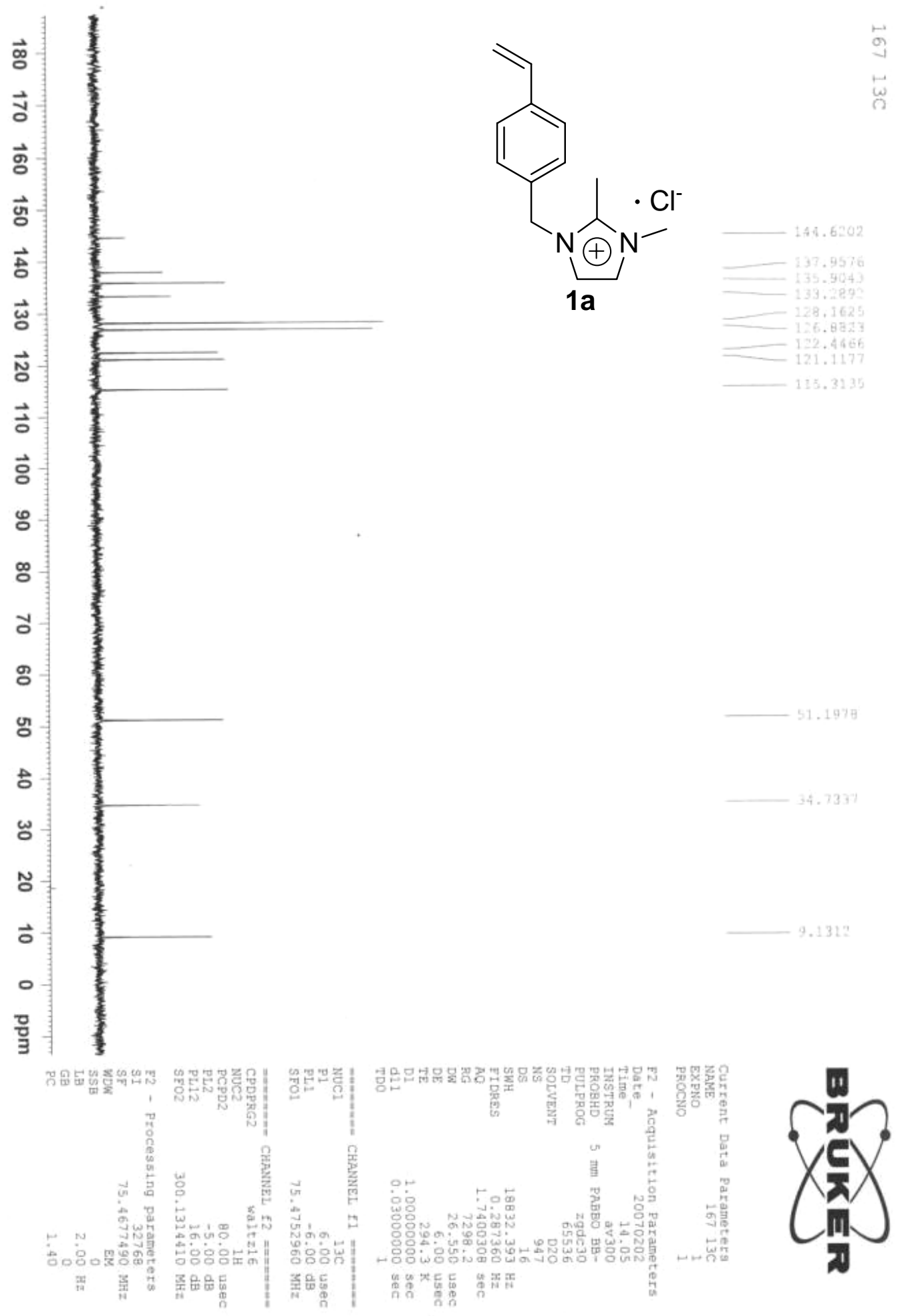




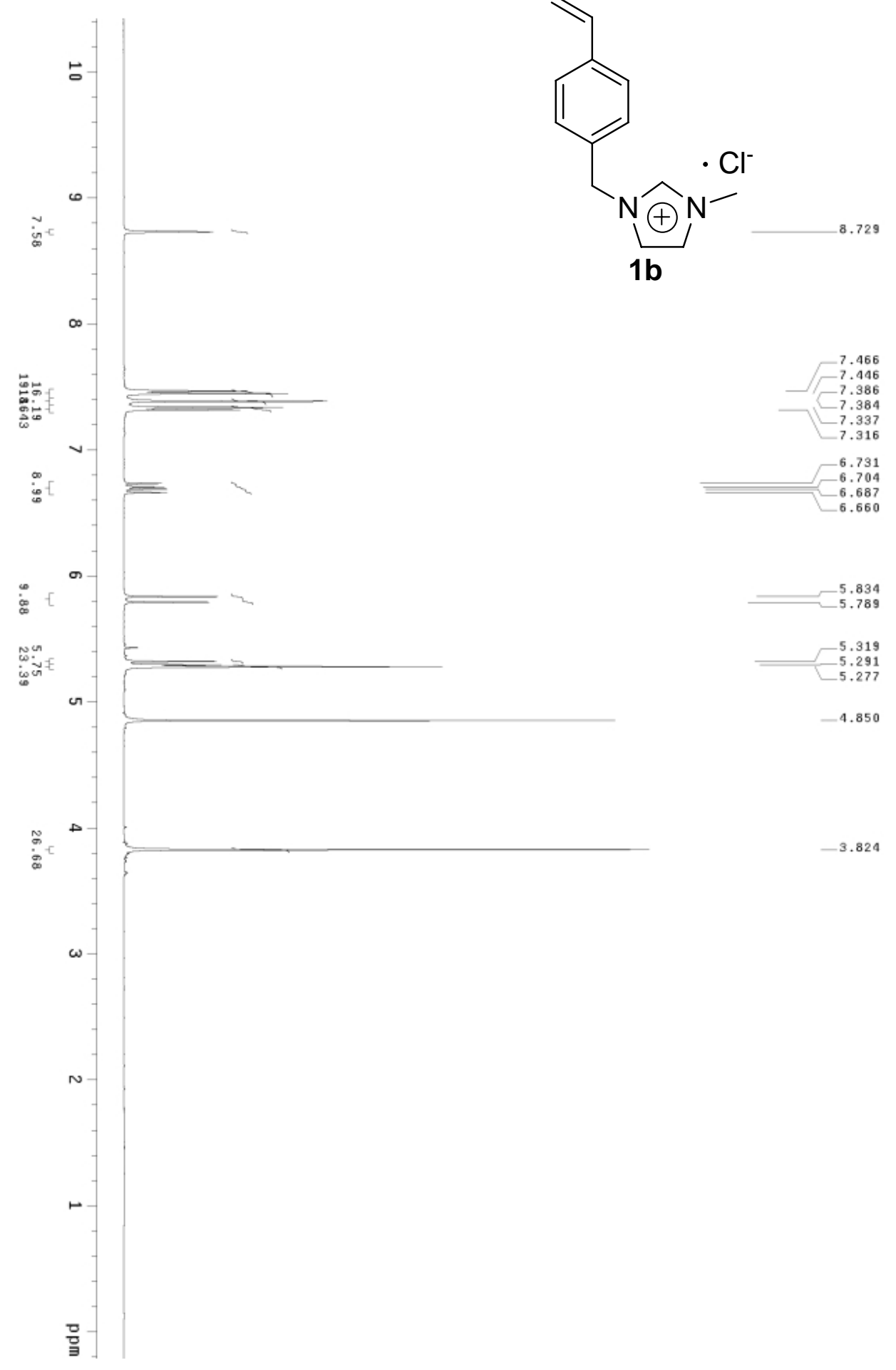

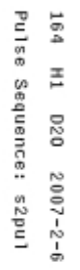




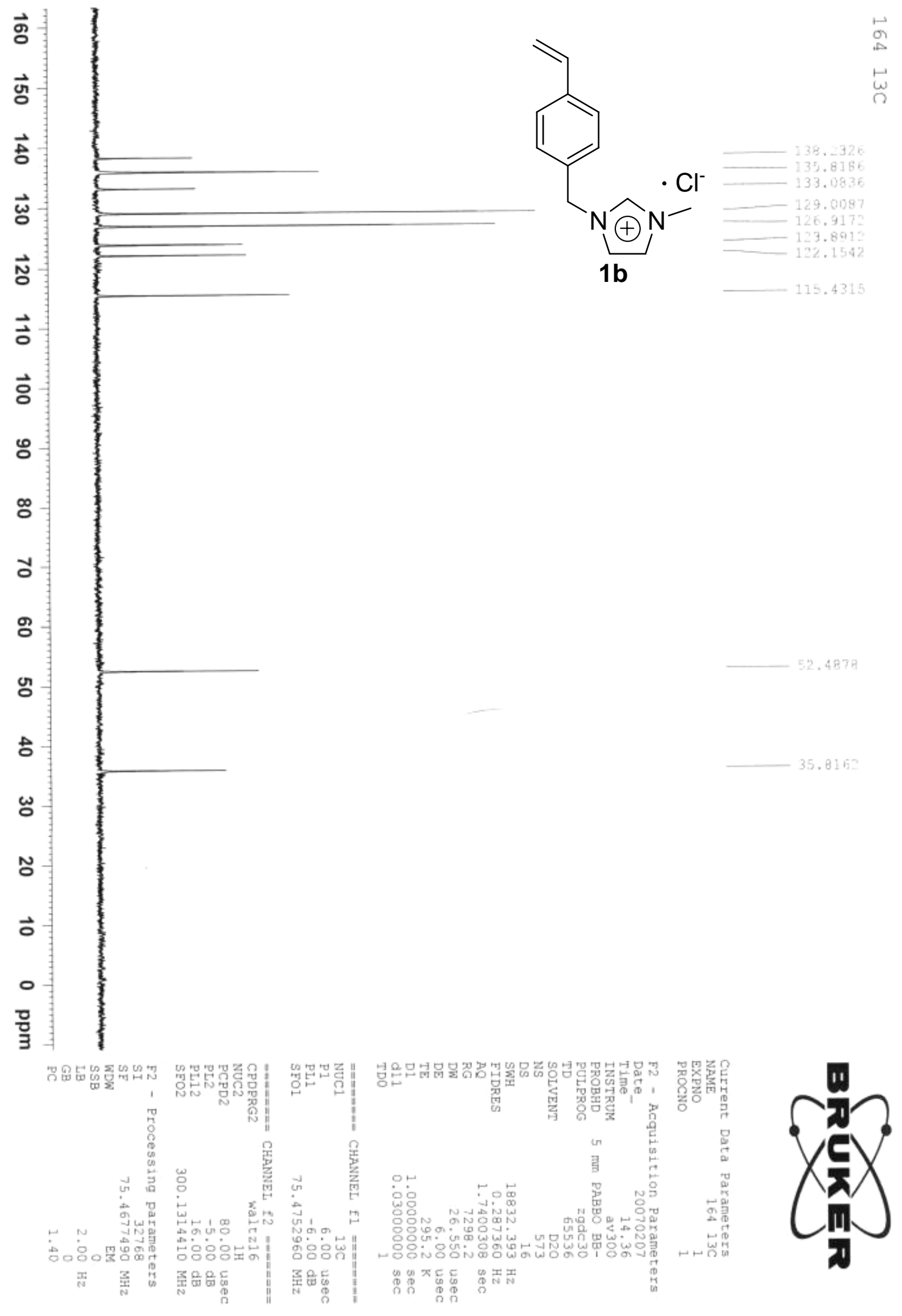




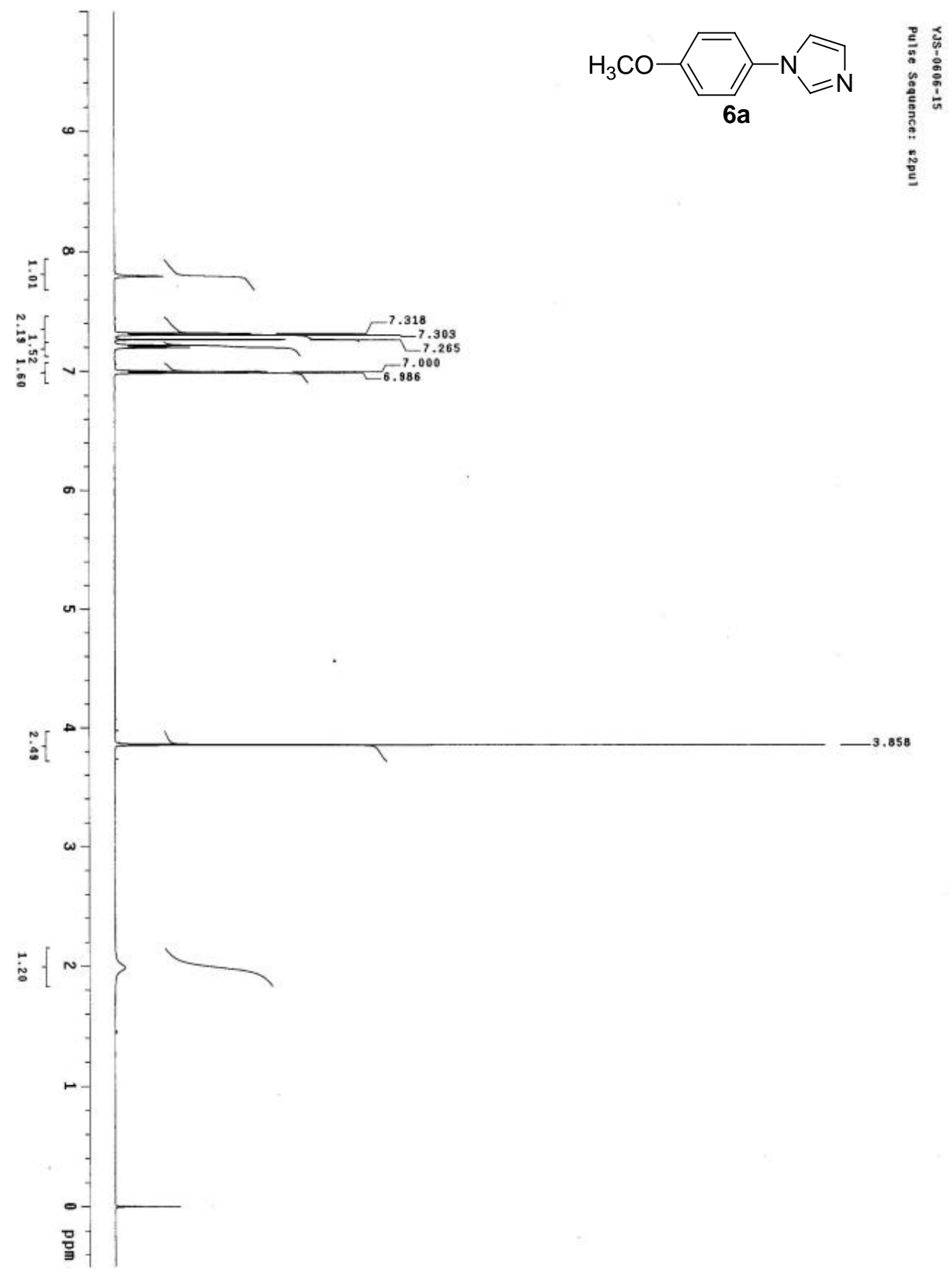




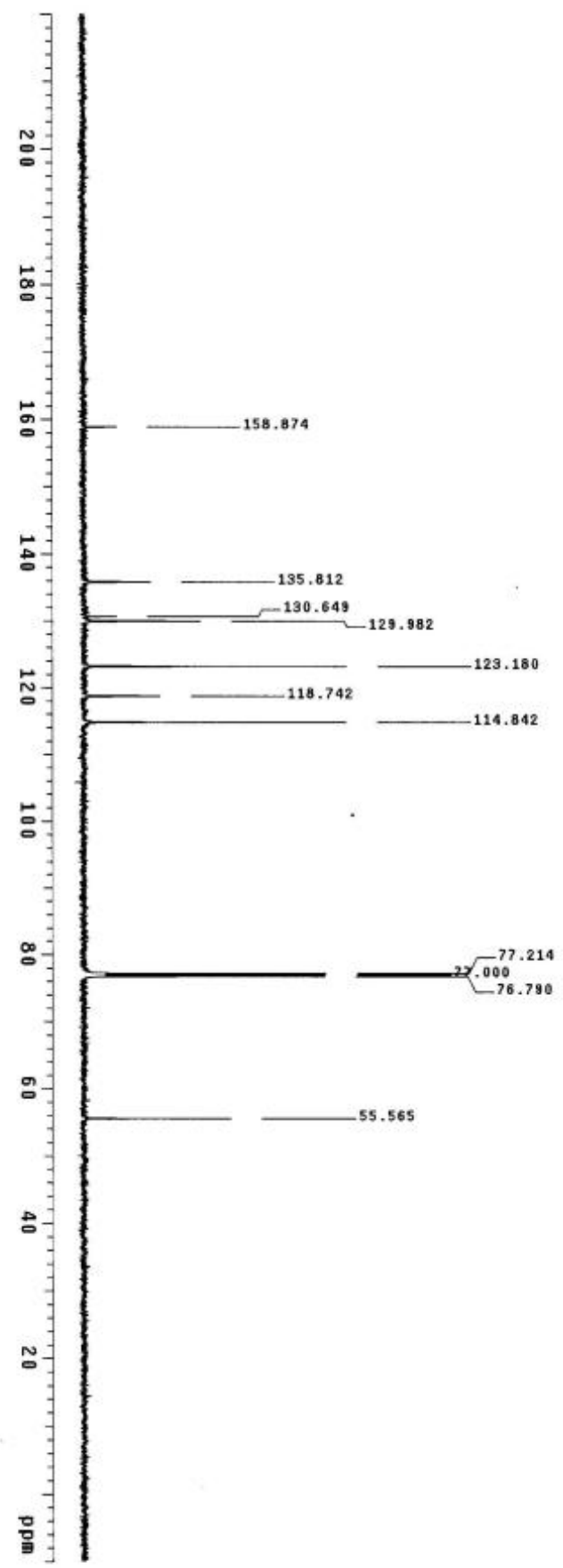




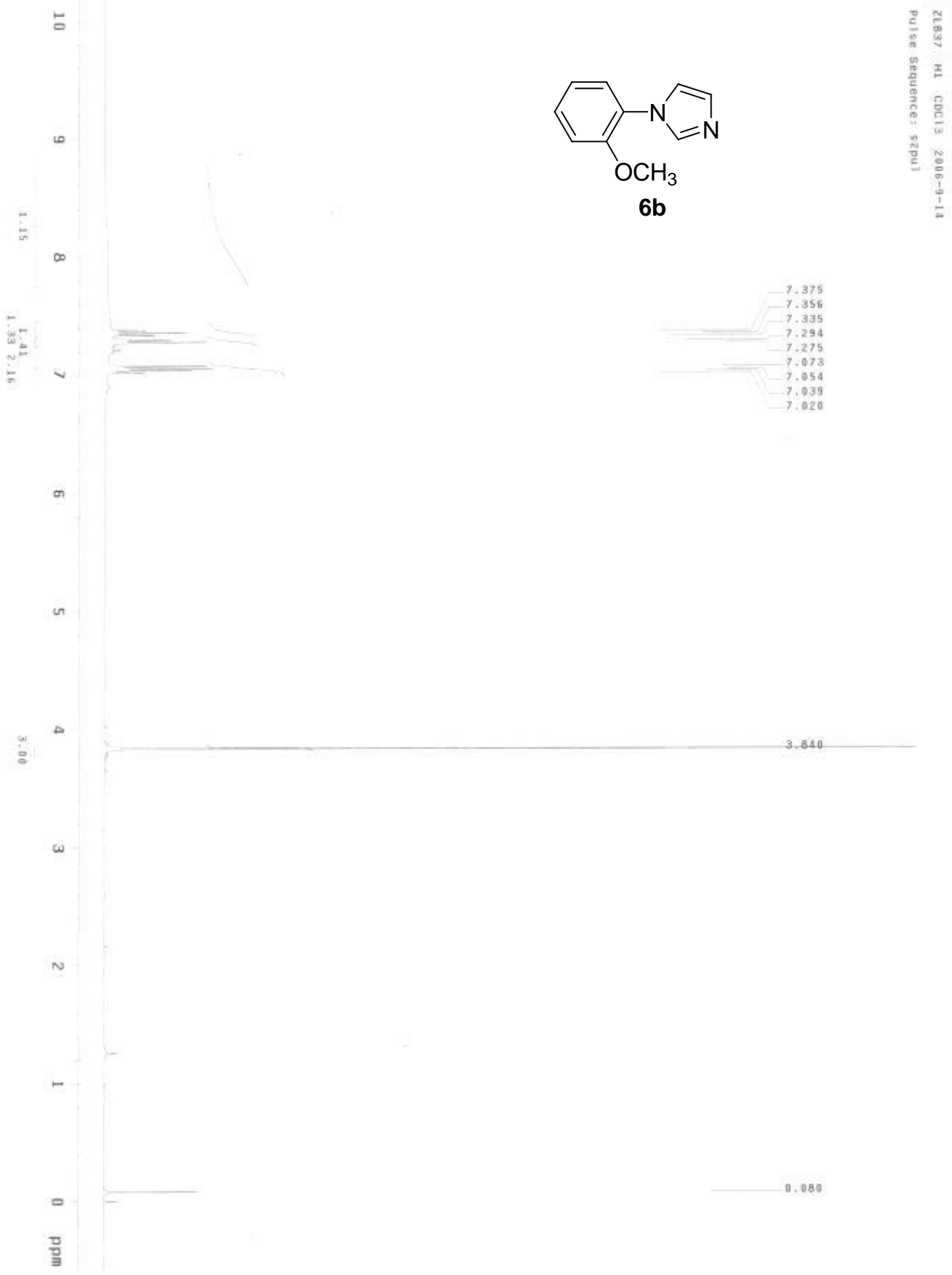



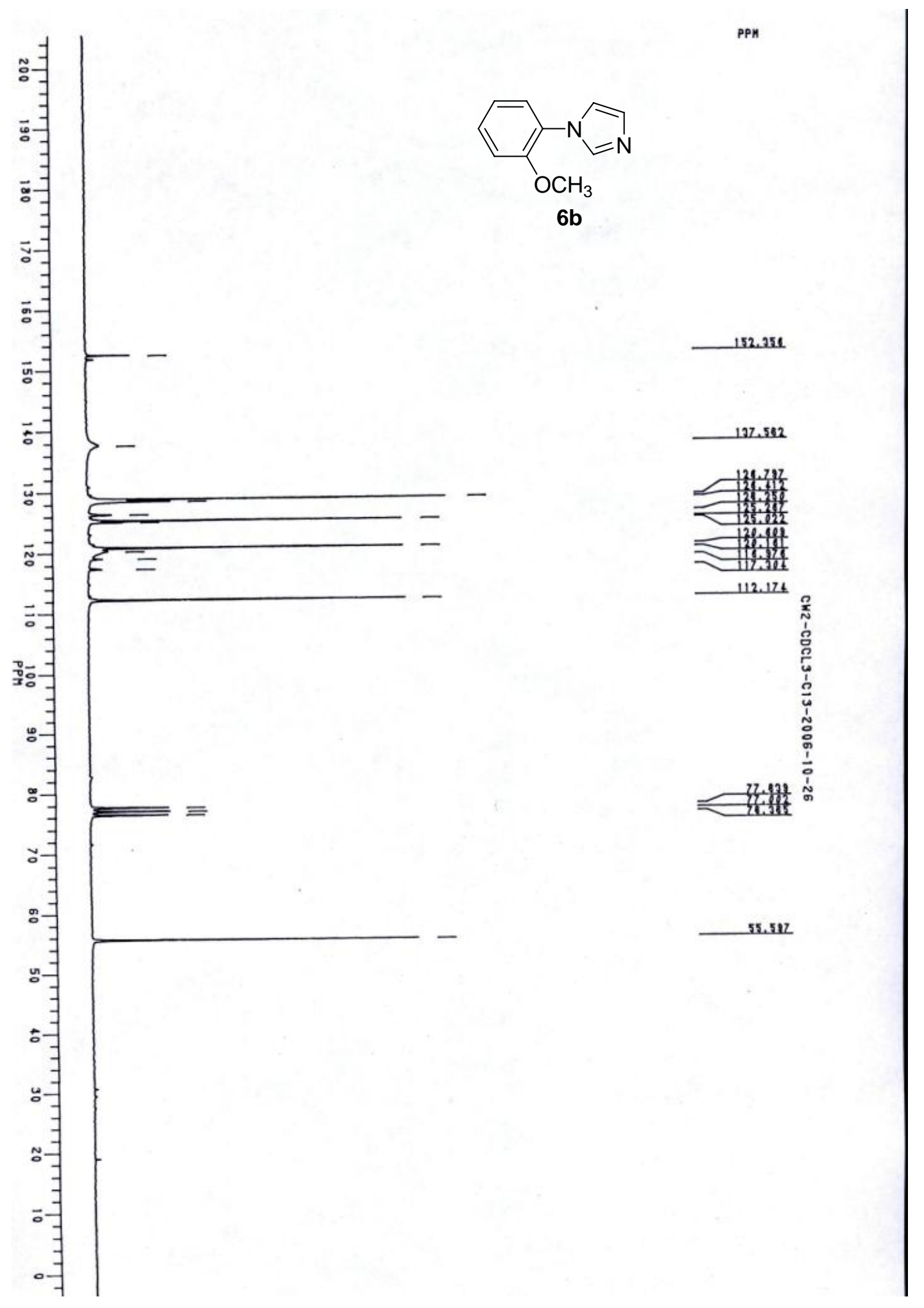


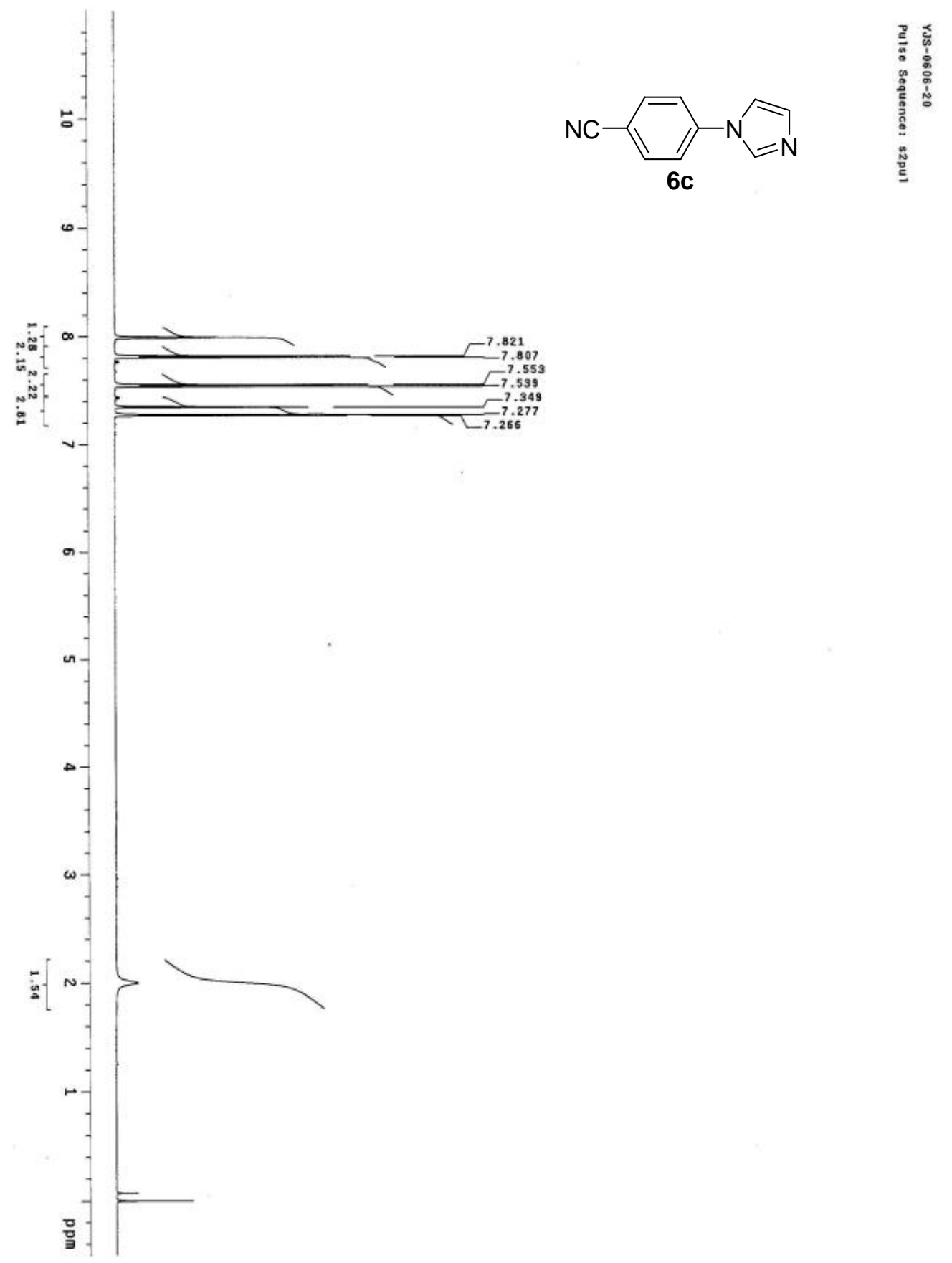




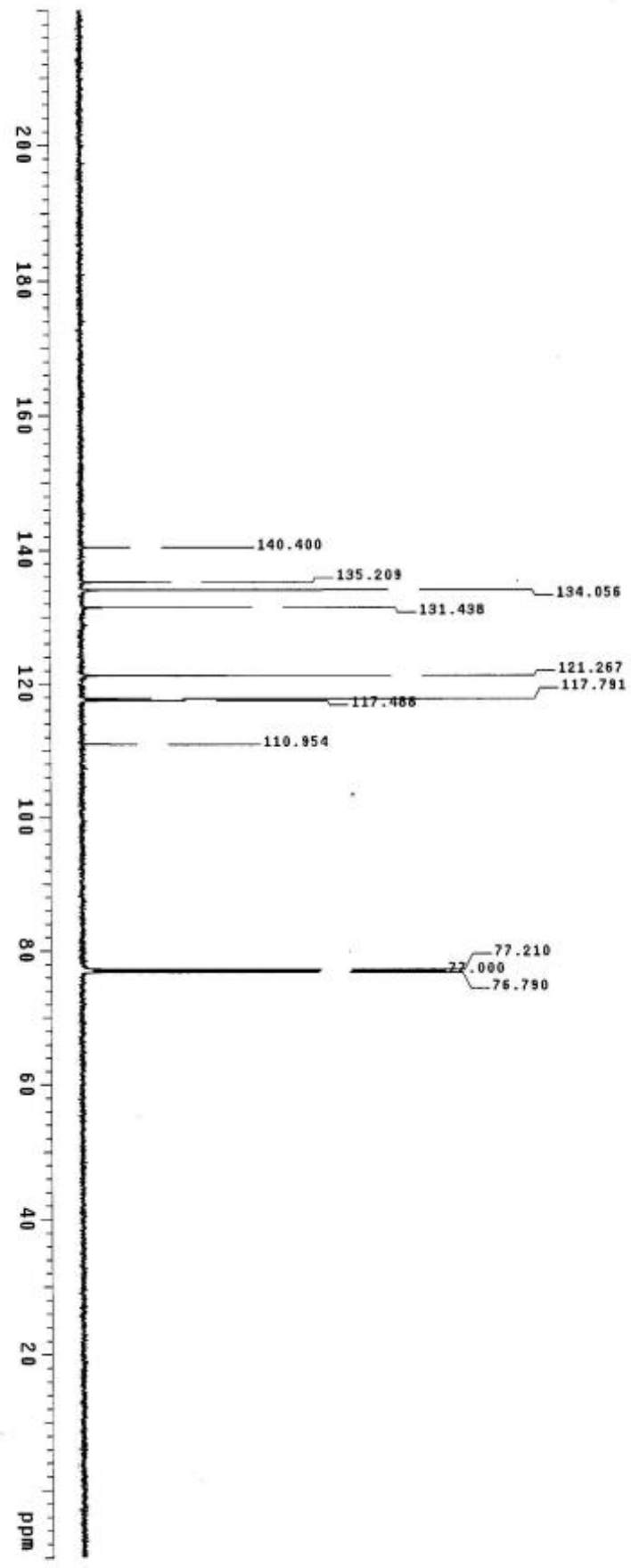




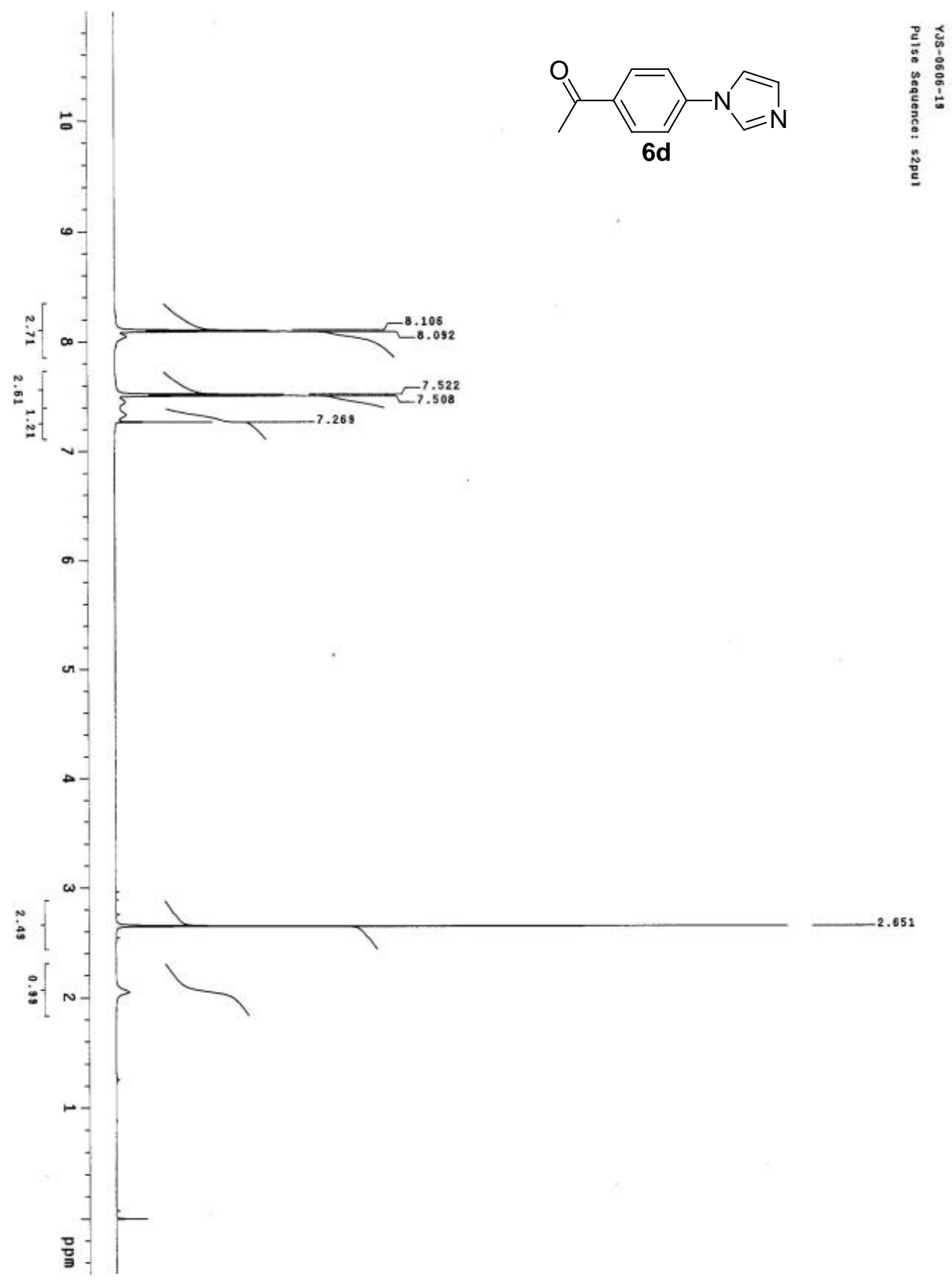




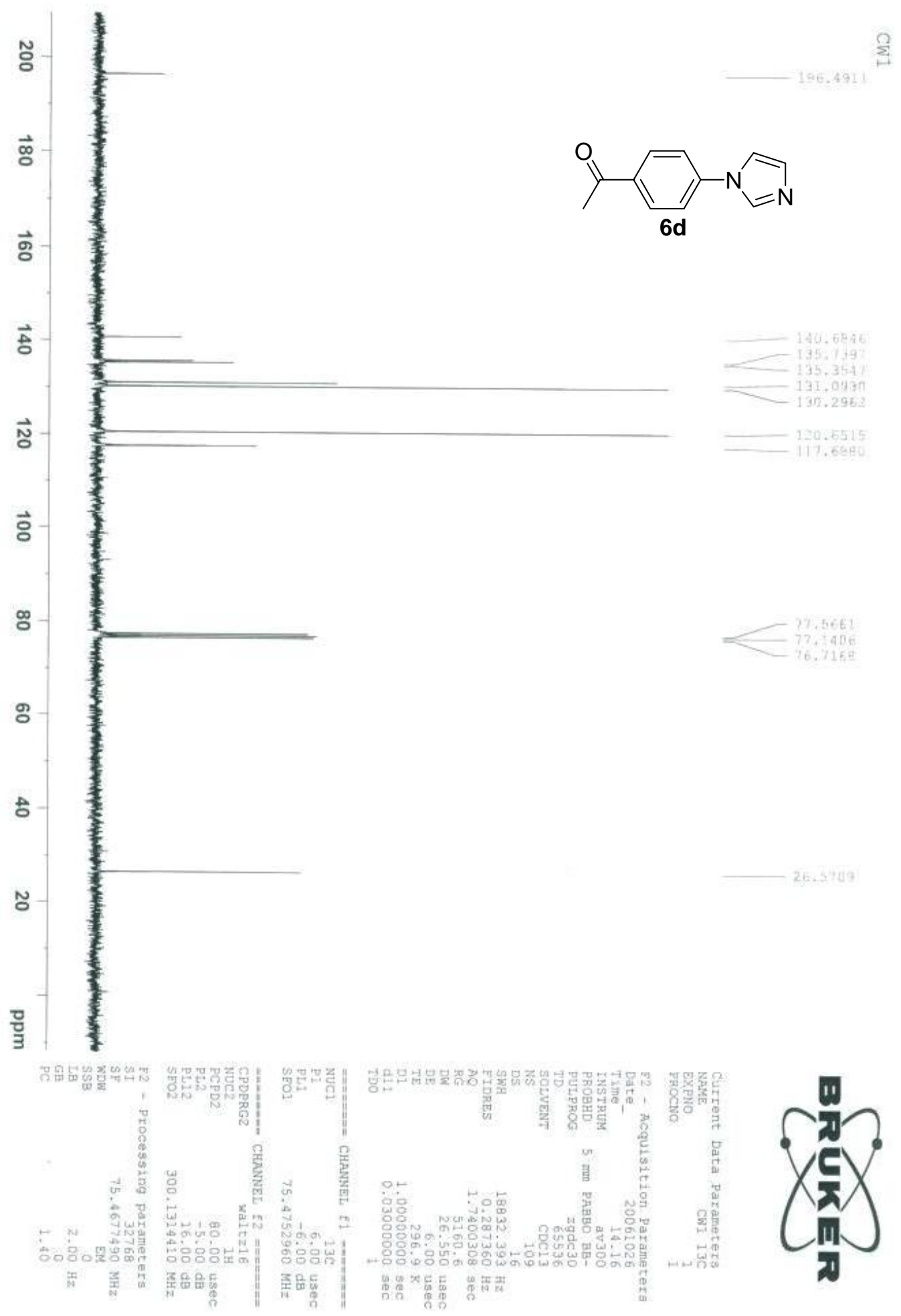



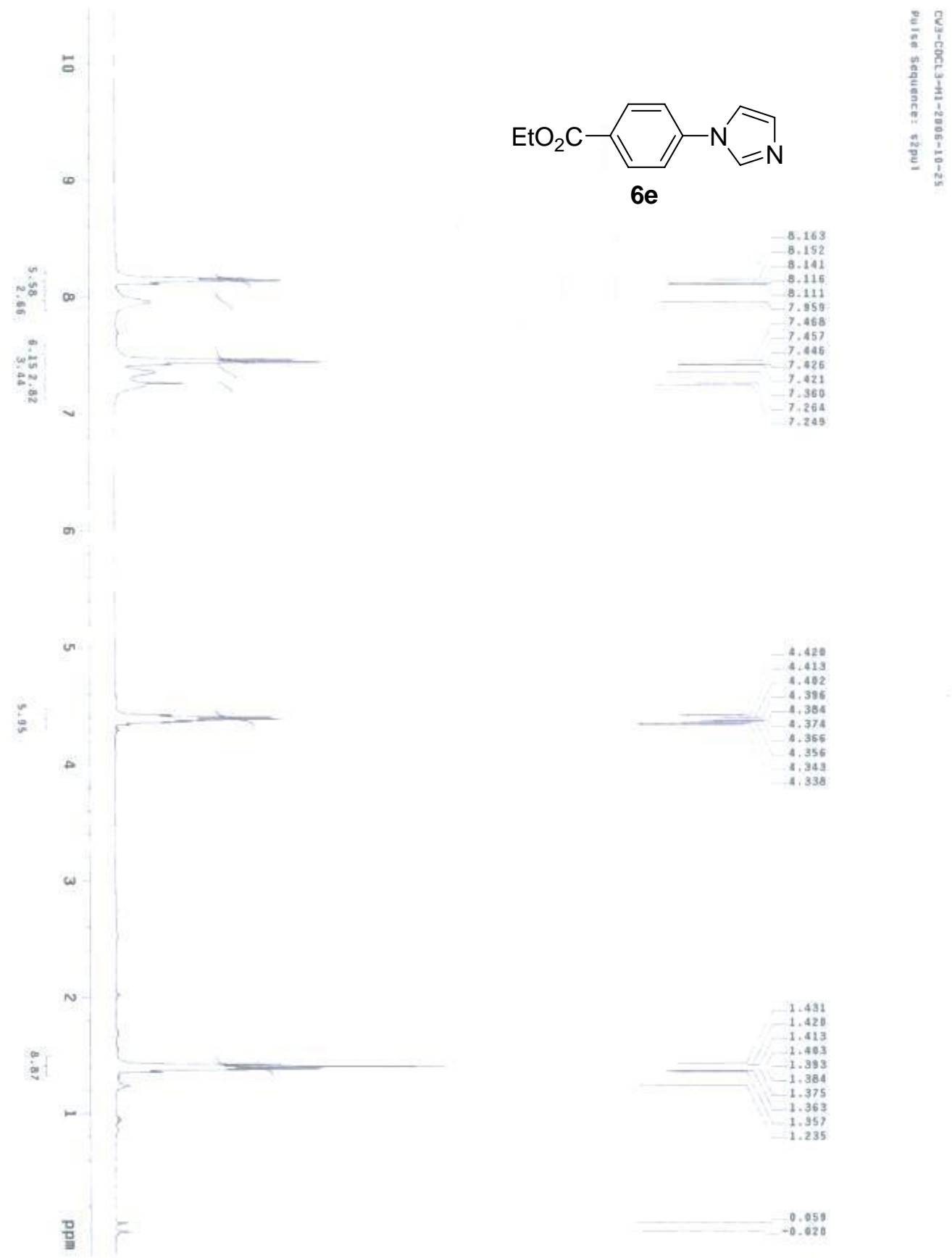


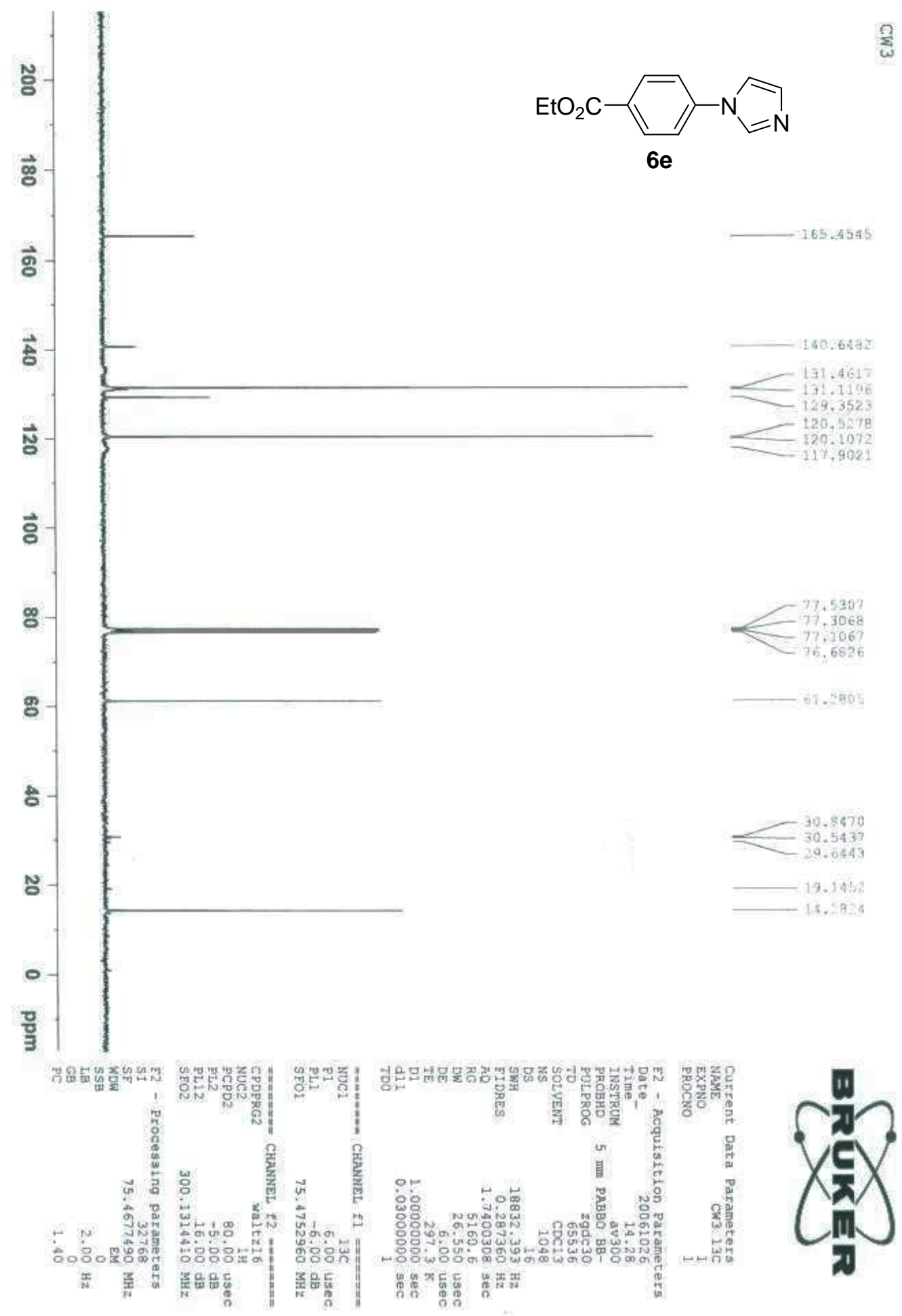




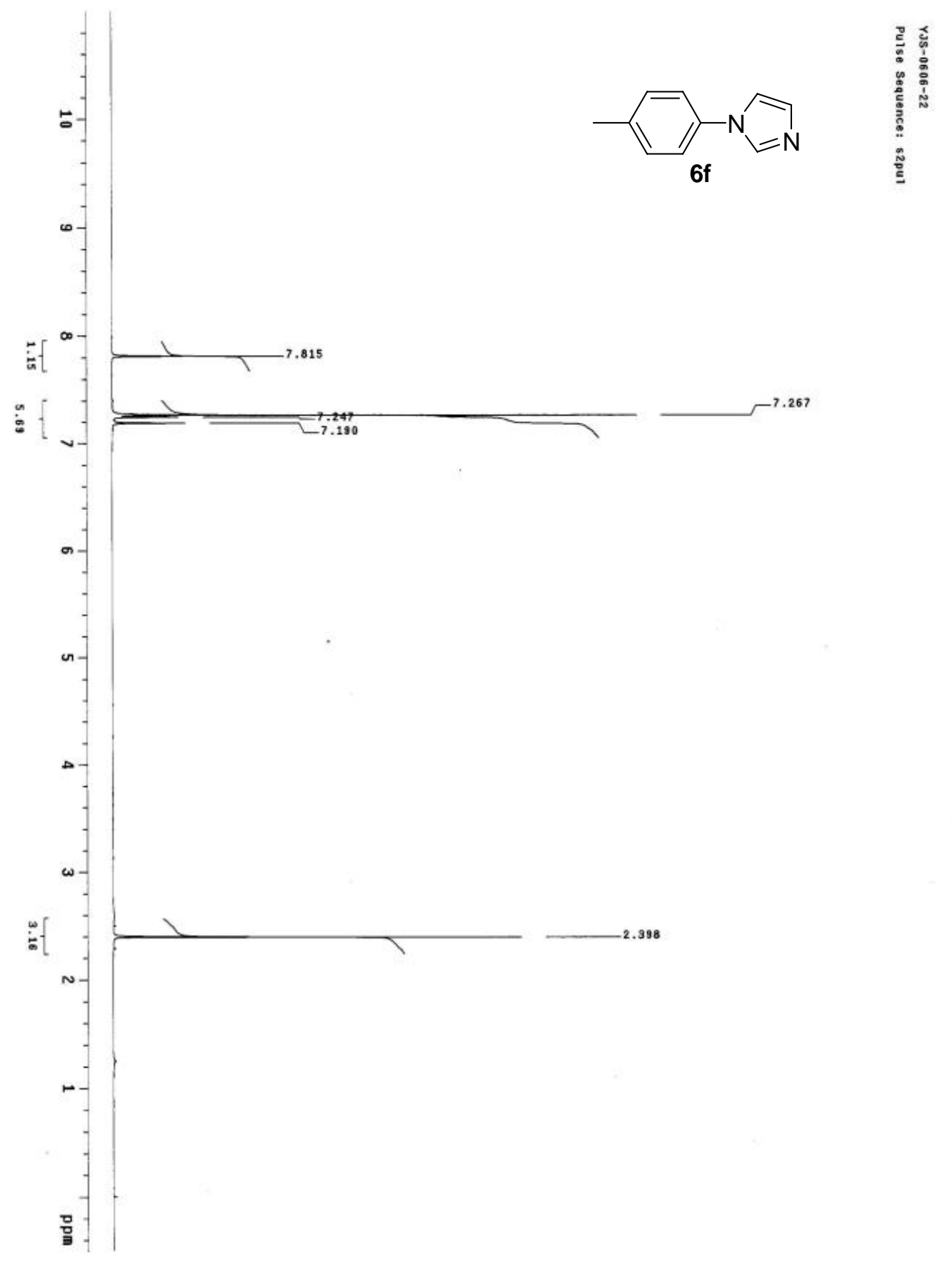



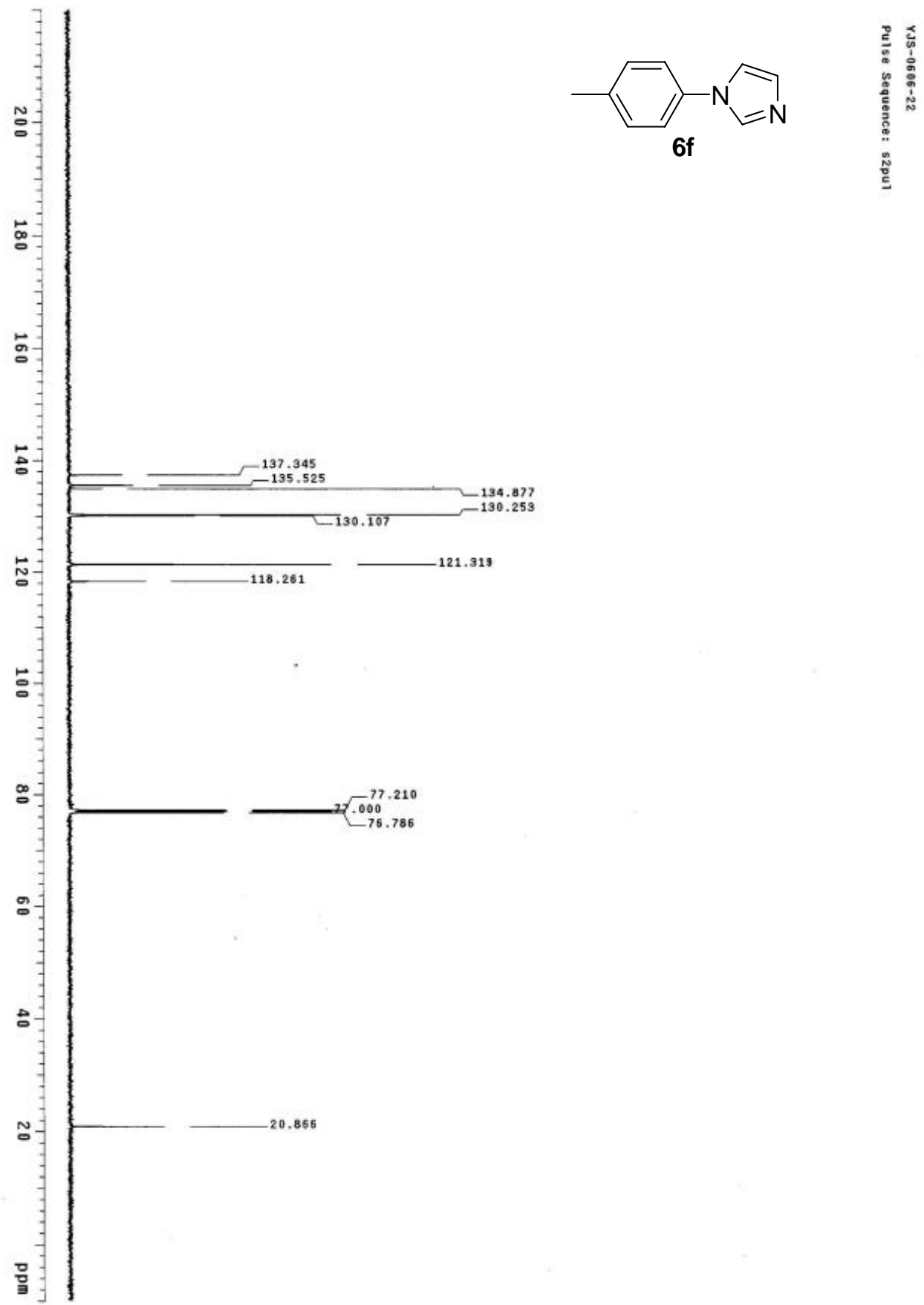


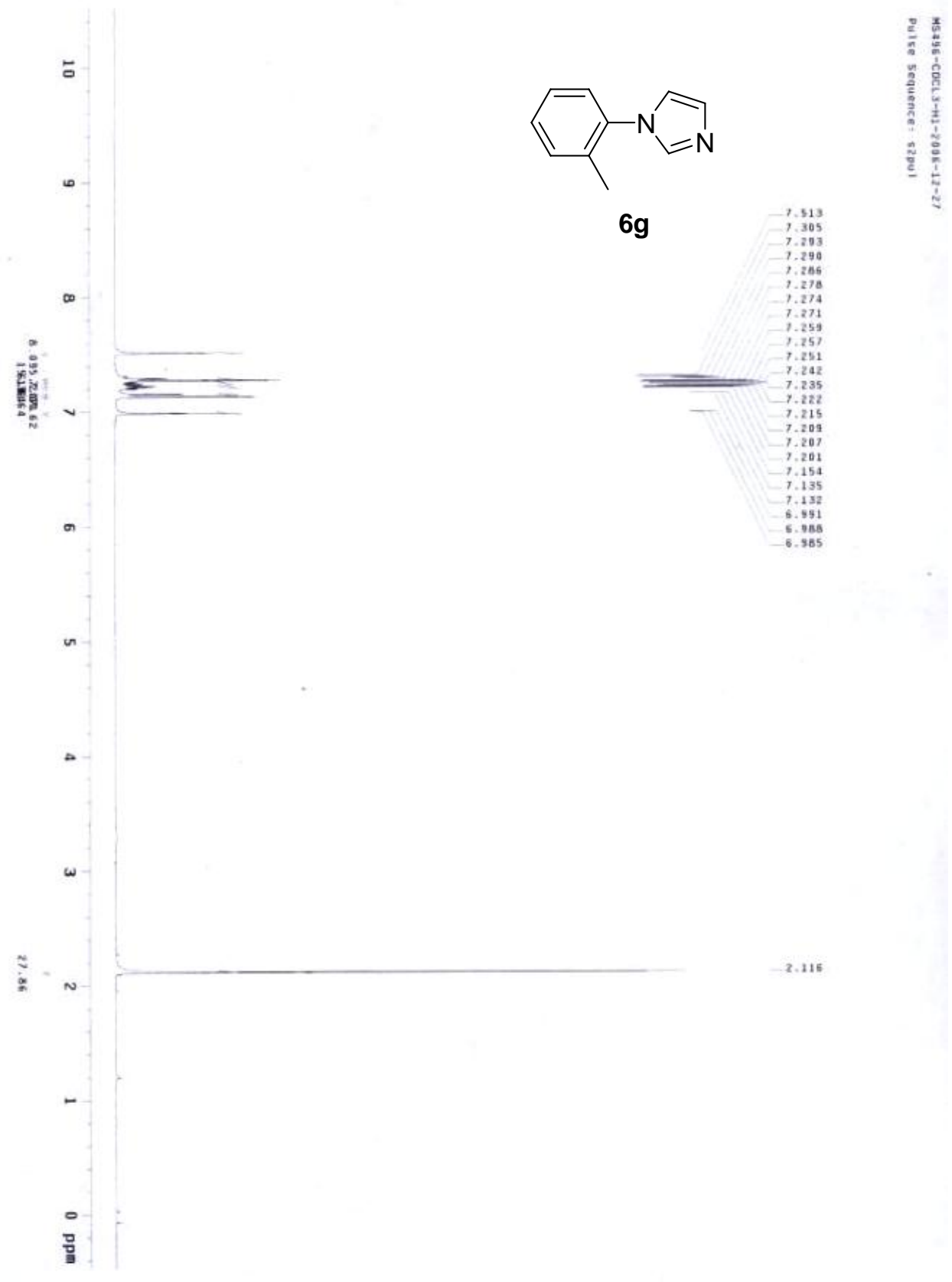



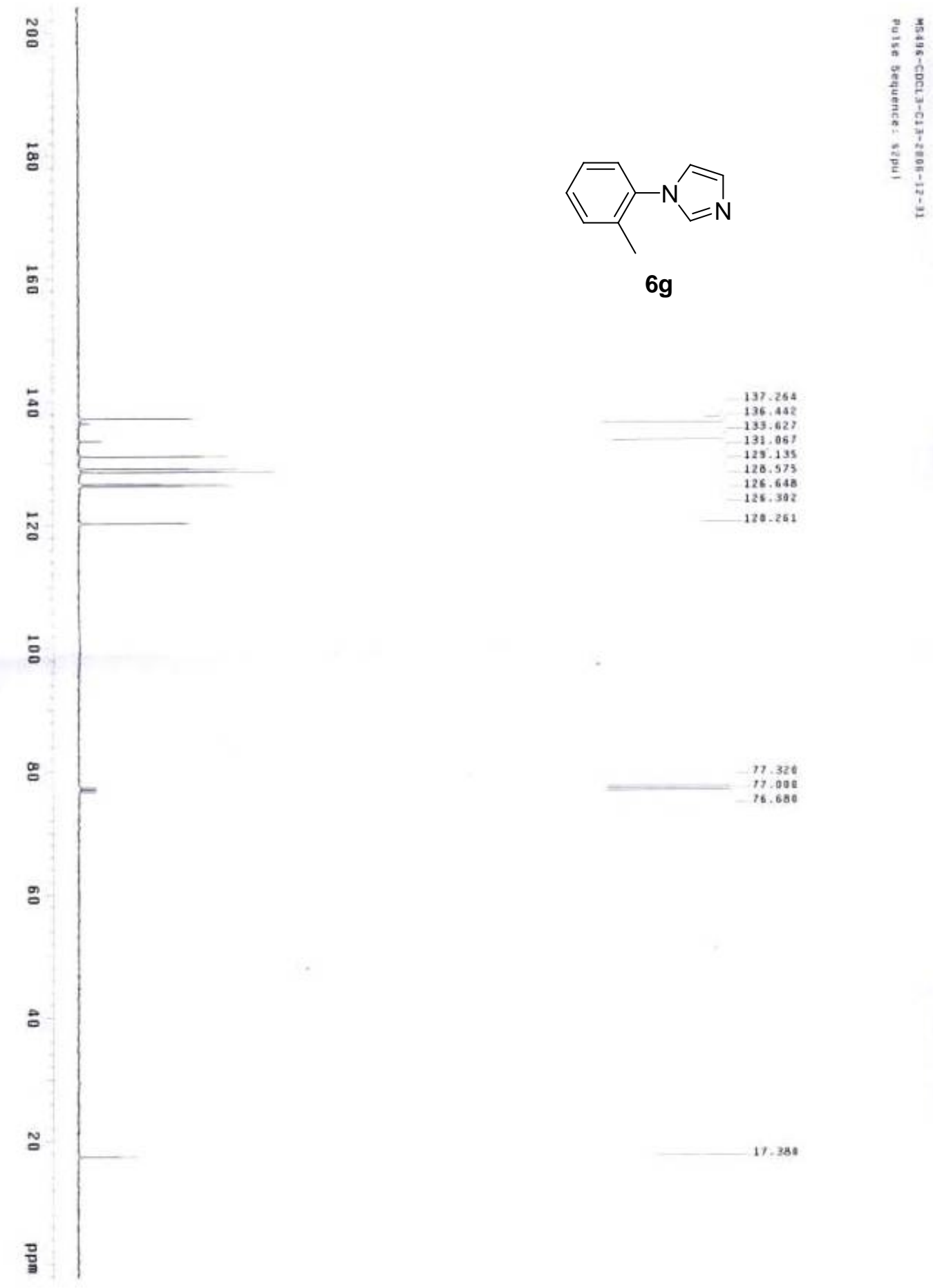

$6 g$

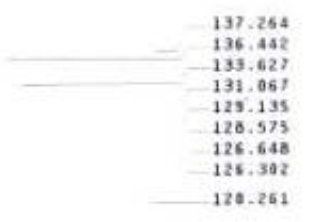

77. 328

7). 700

17. 384 


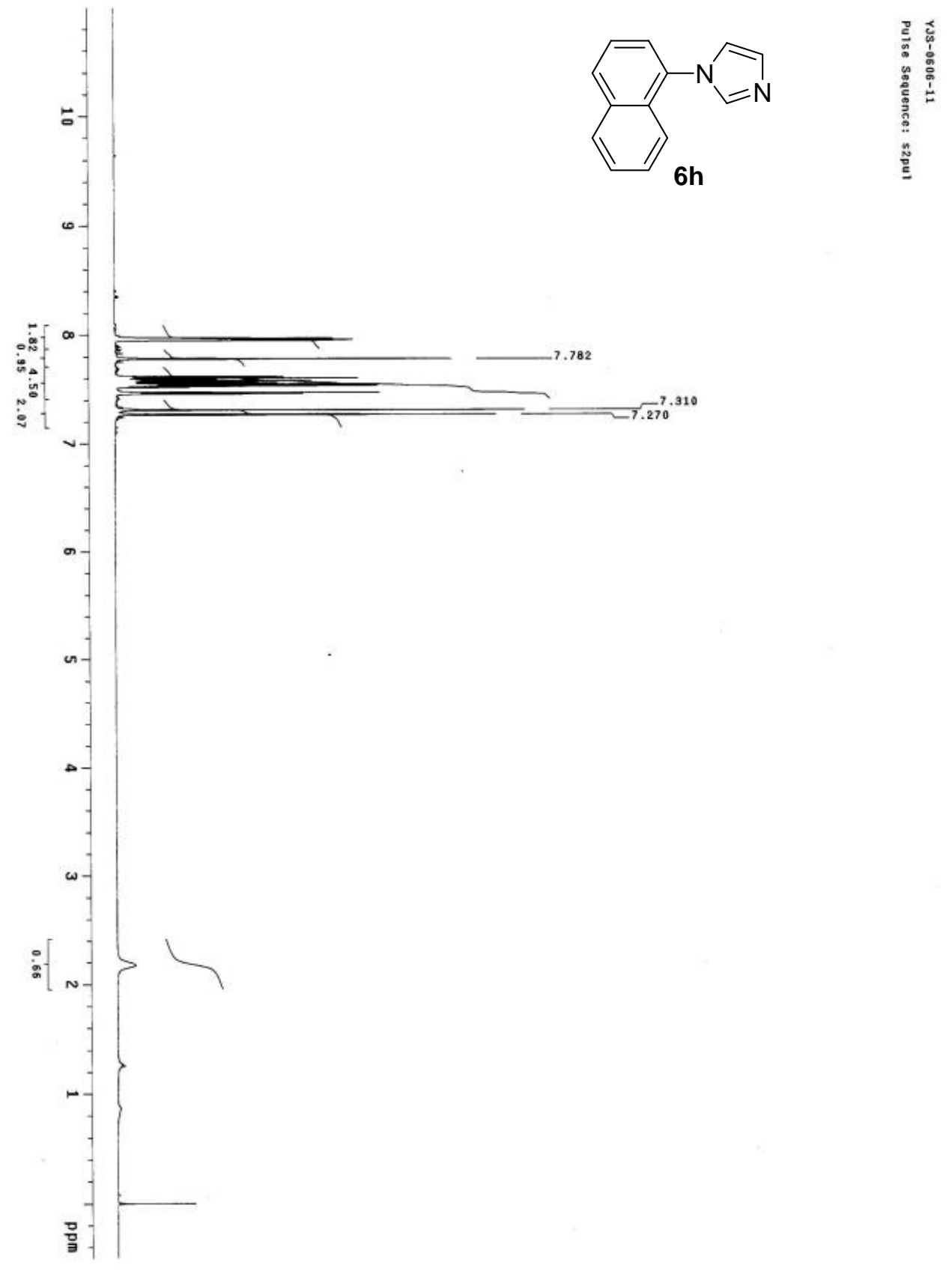



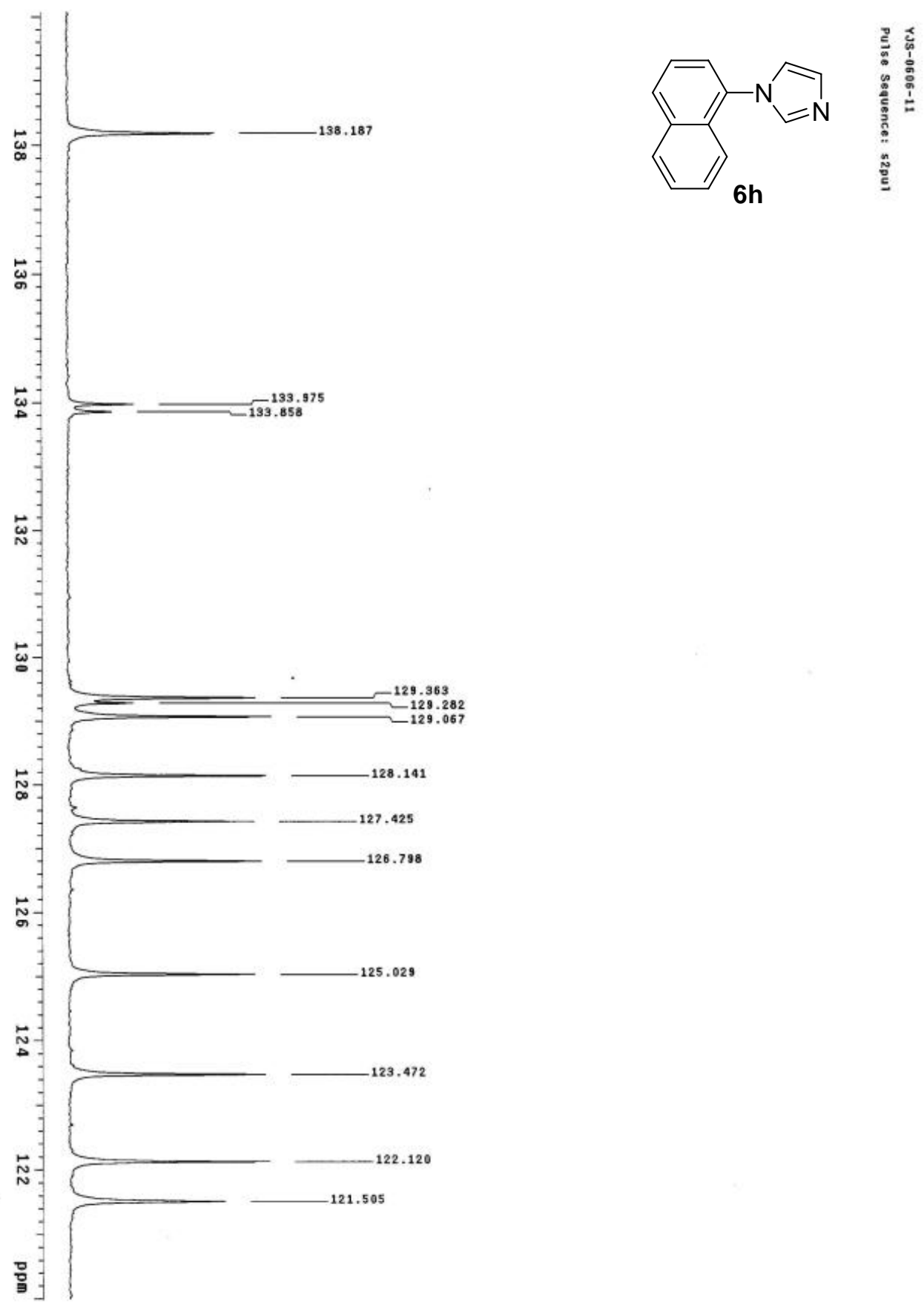


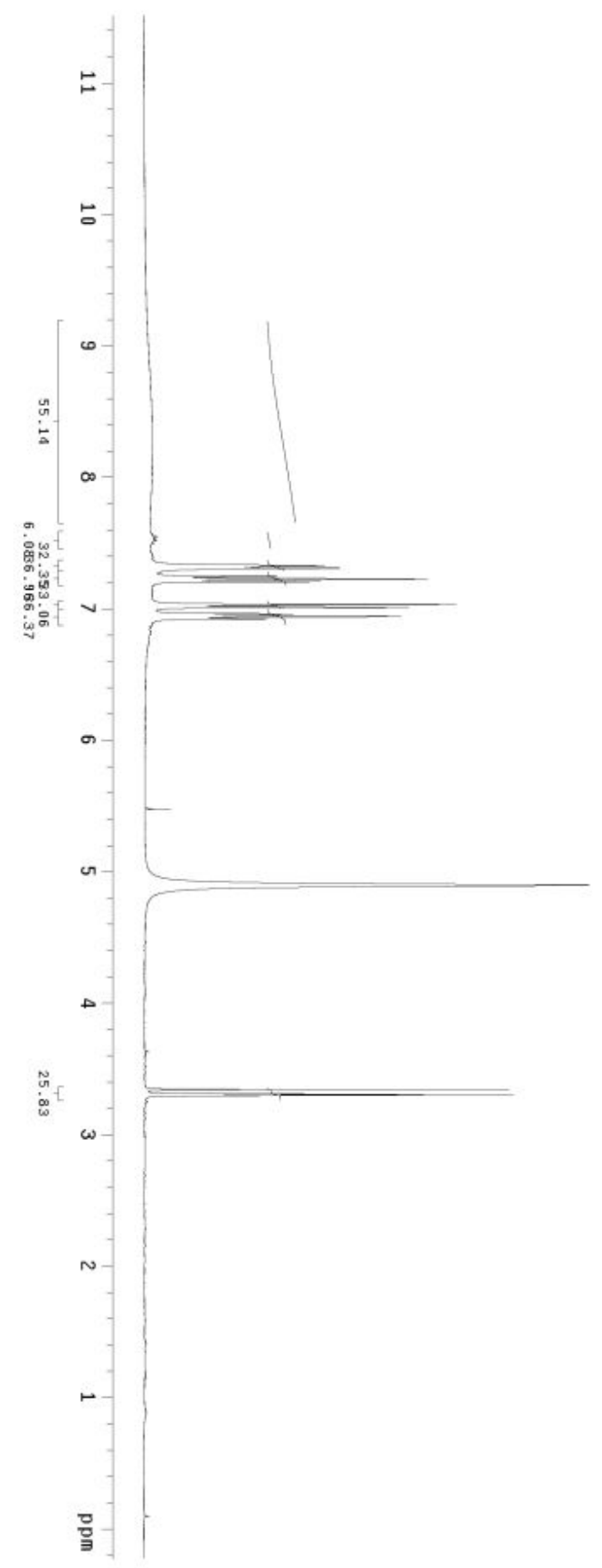

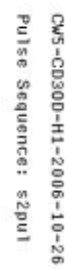

$6 i$
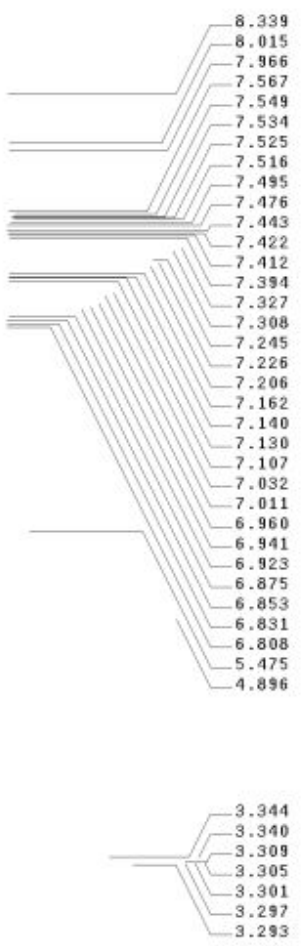


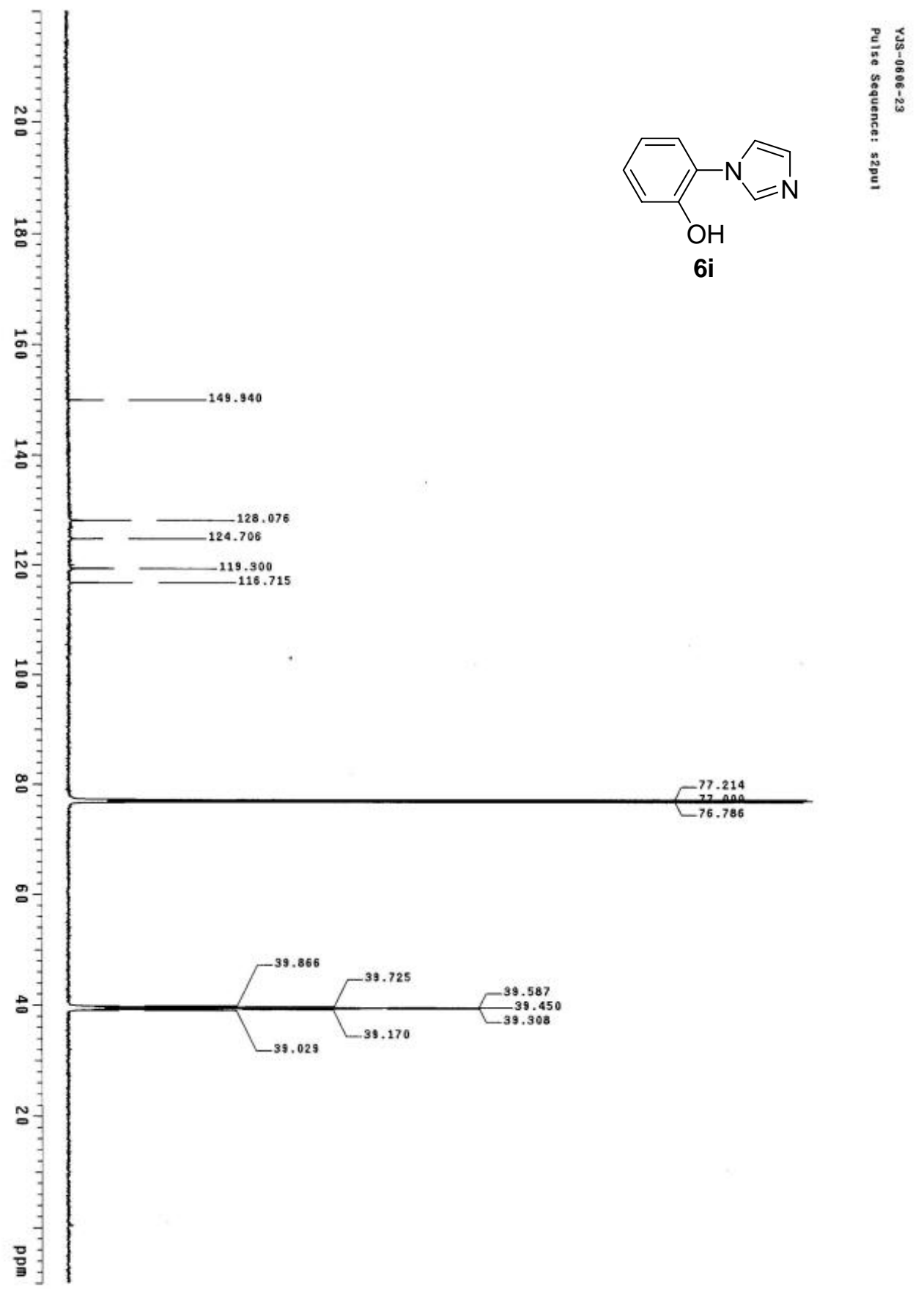




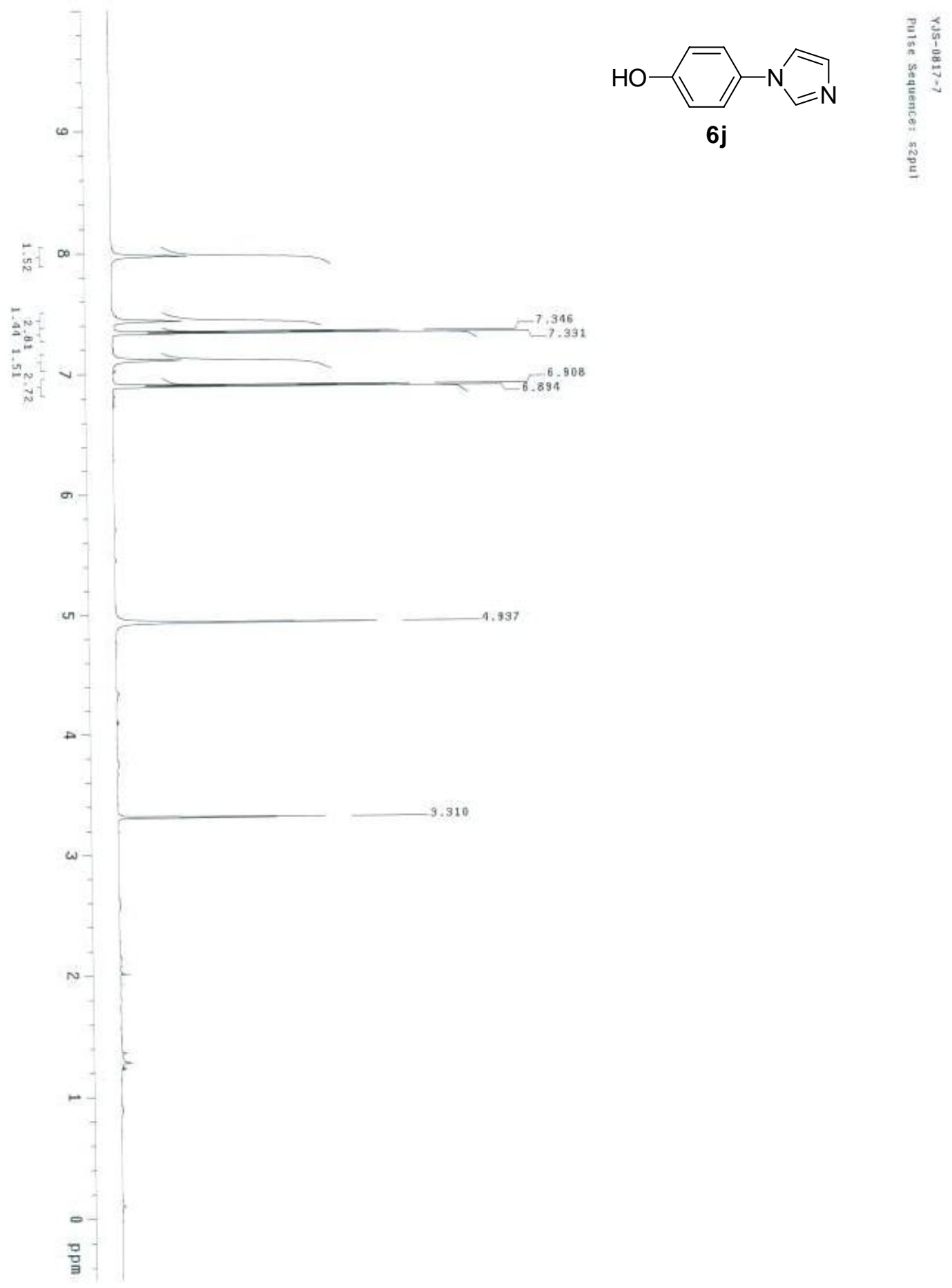




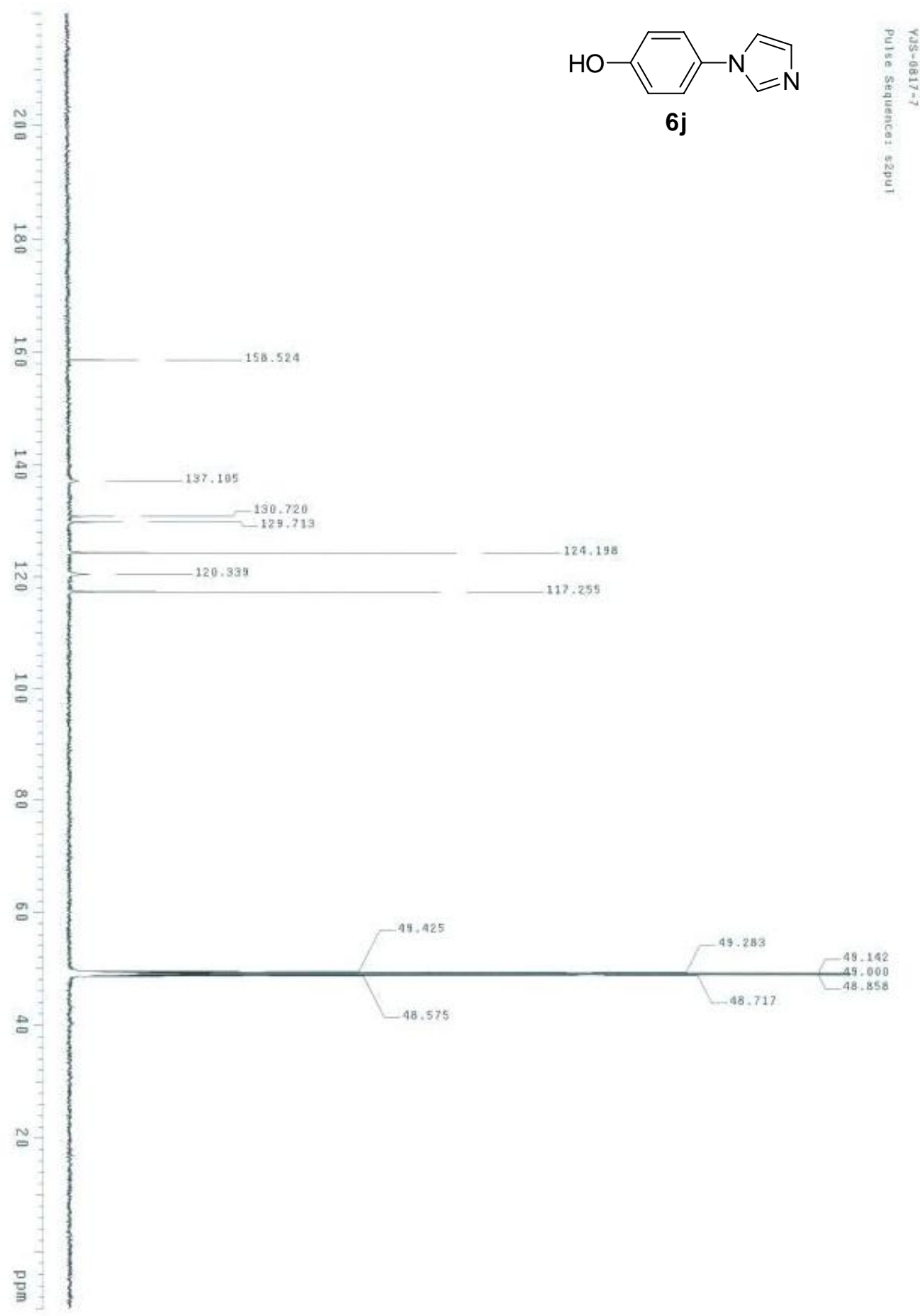




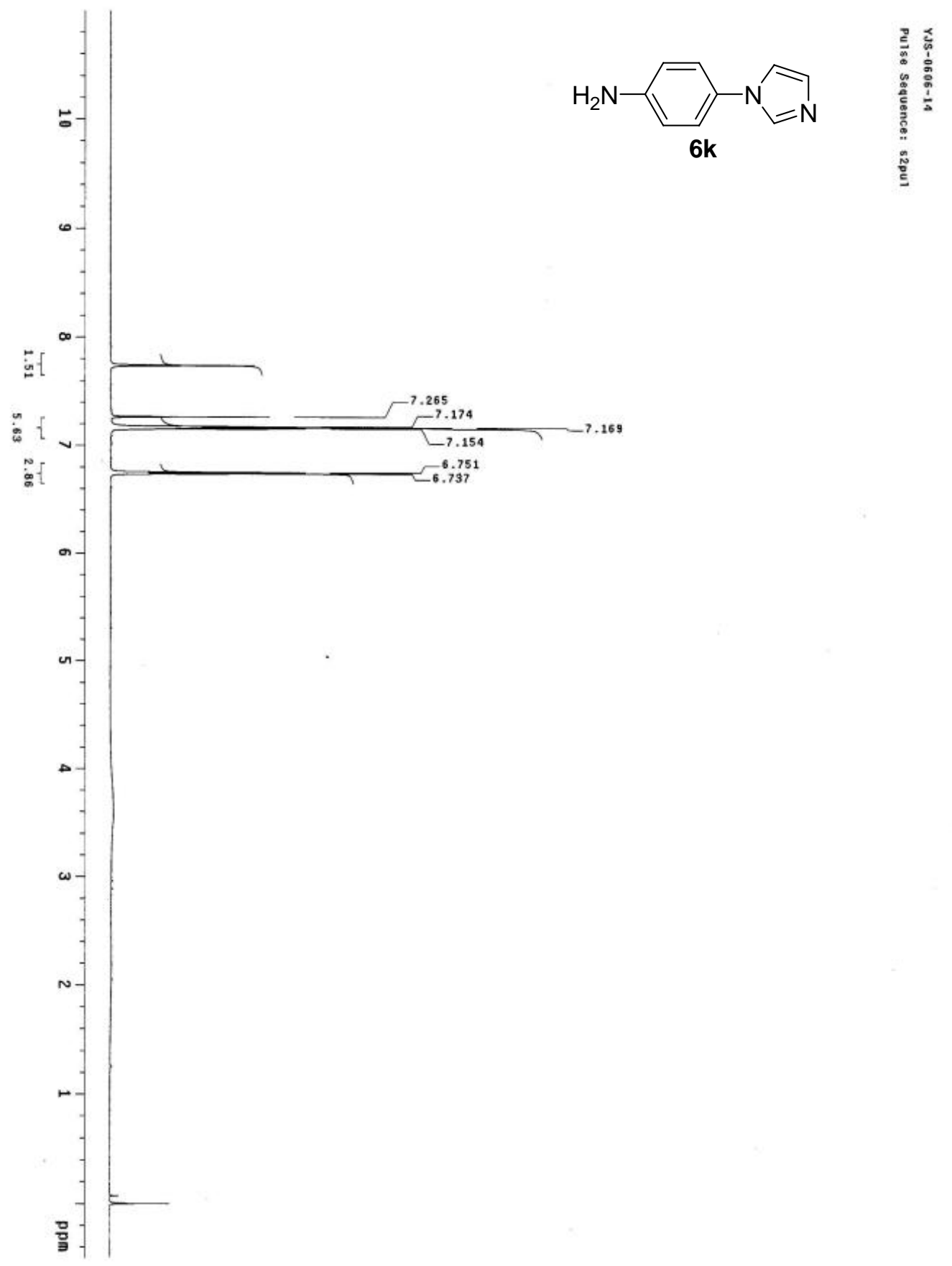



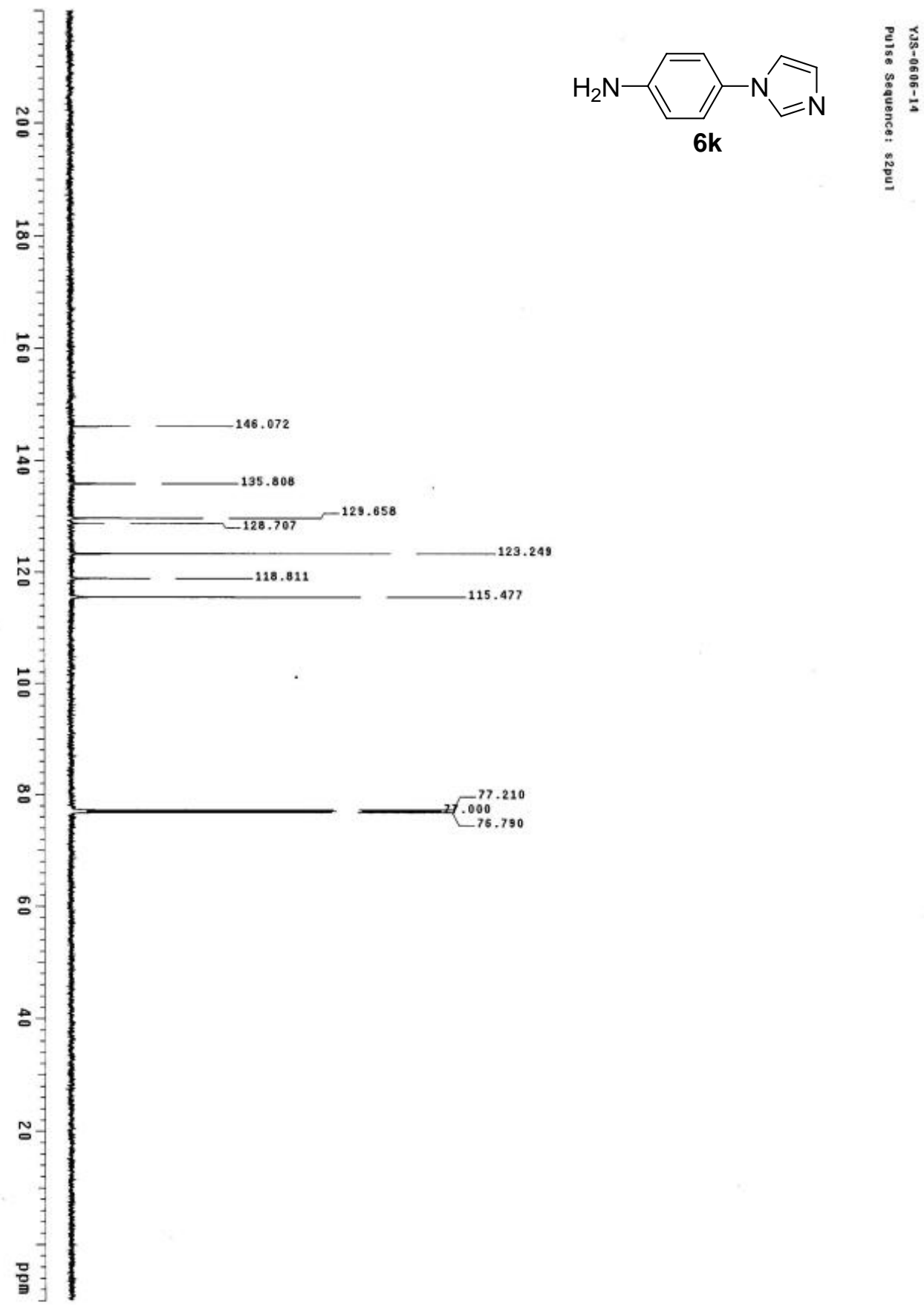

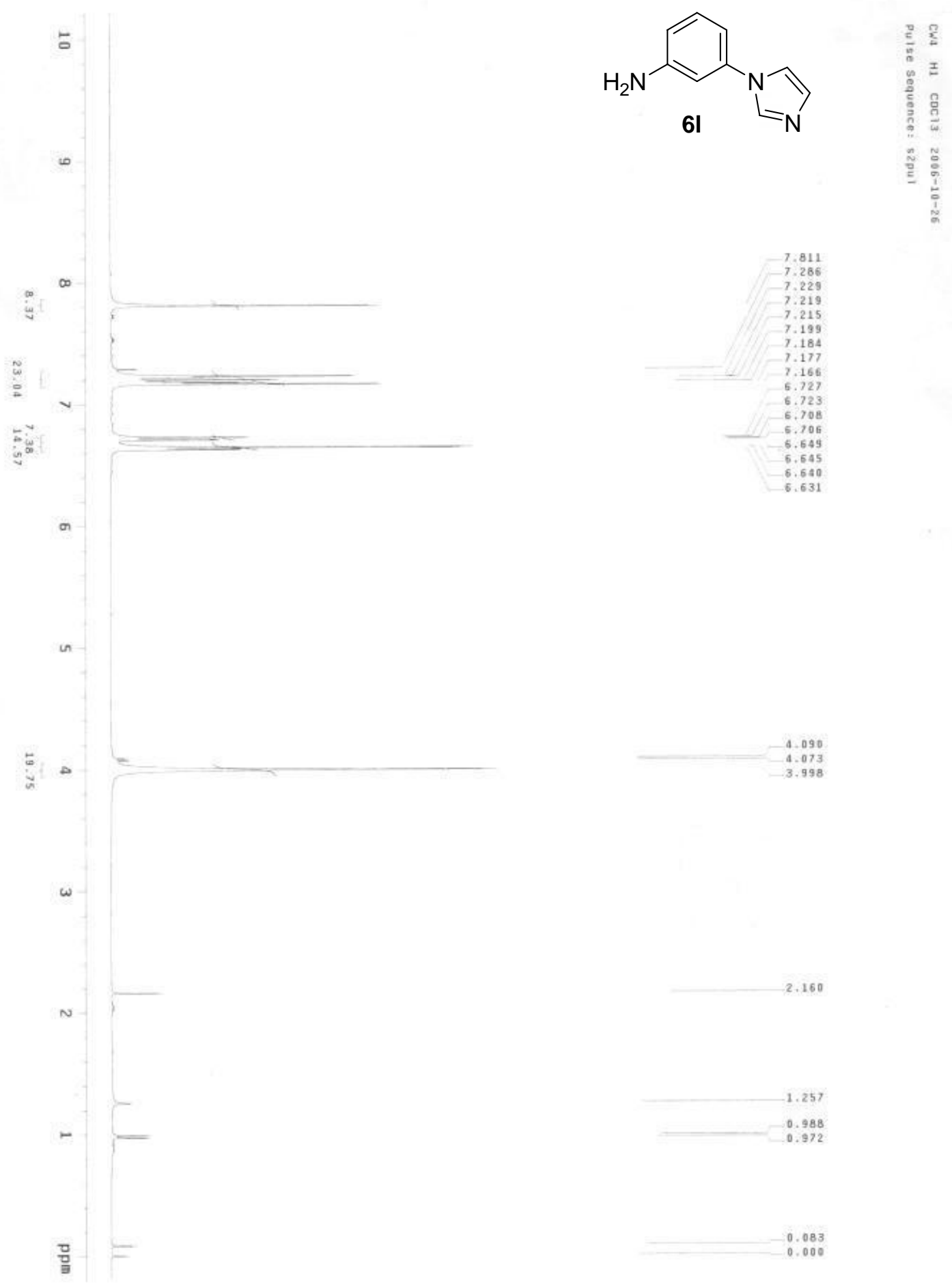


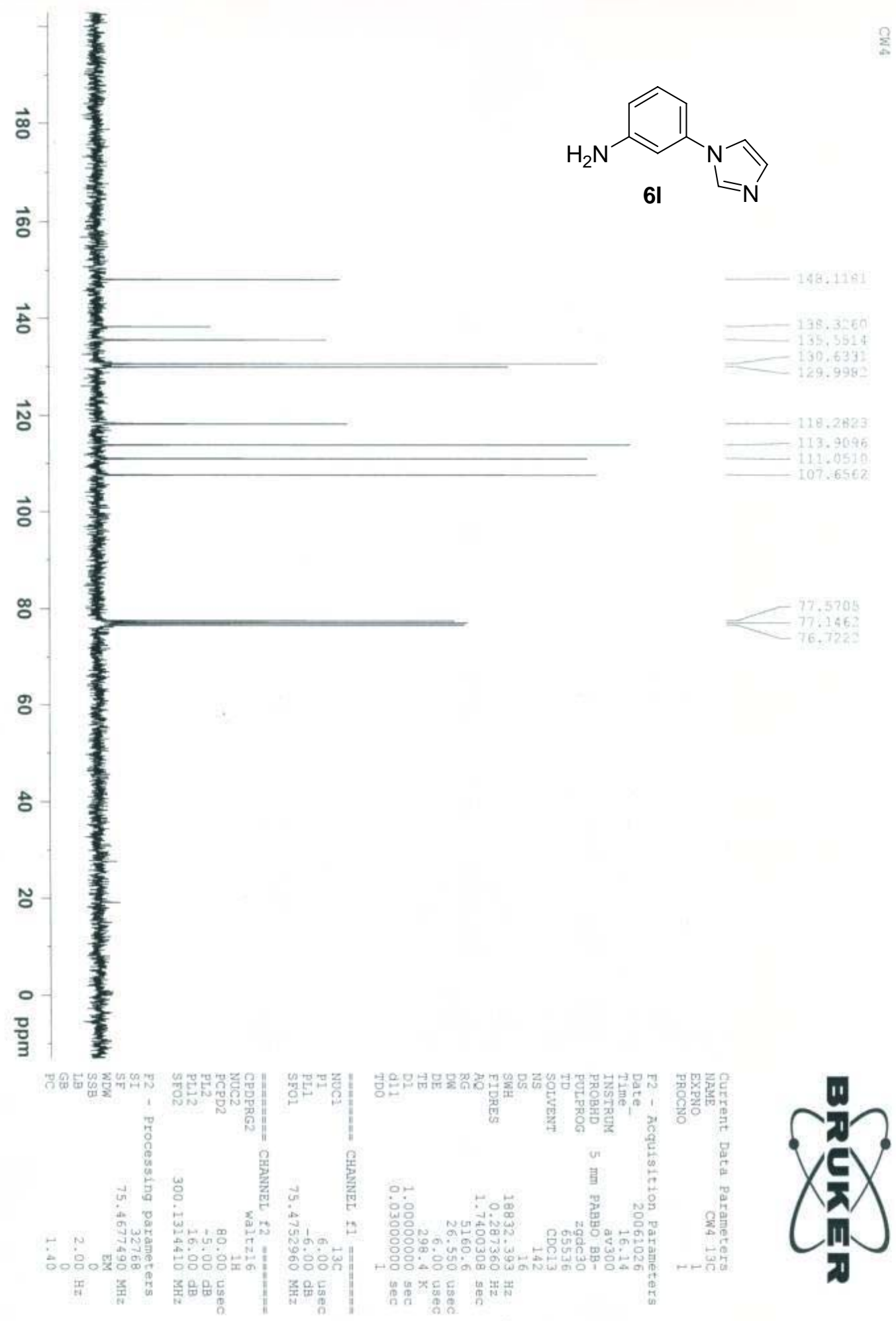




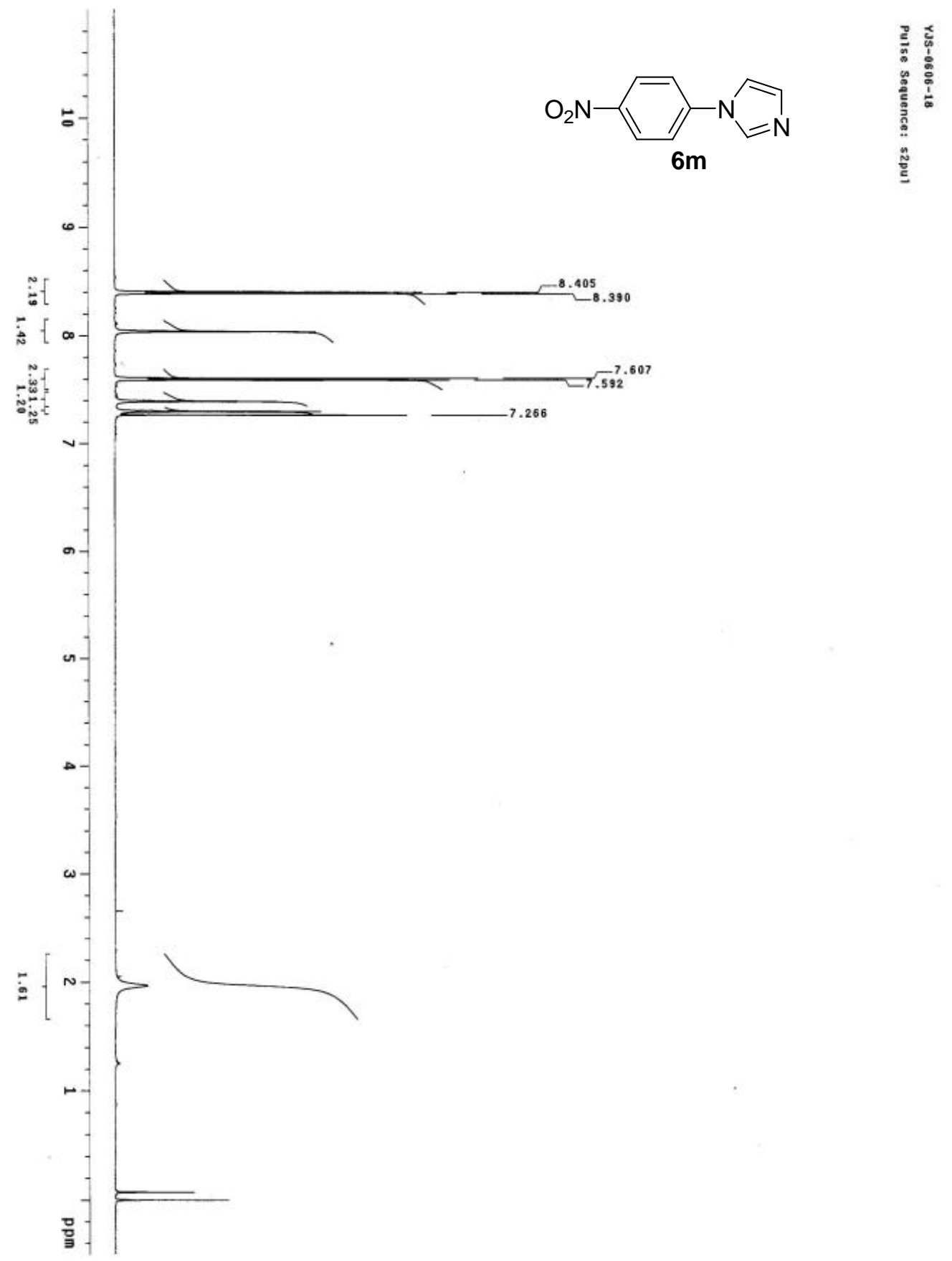




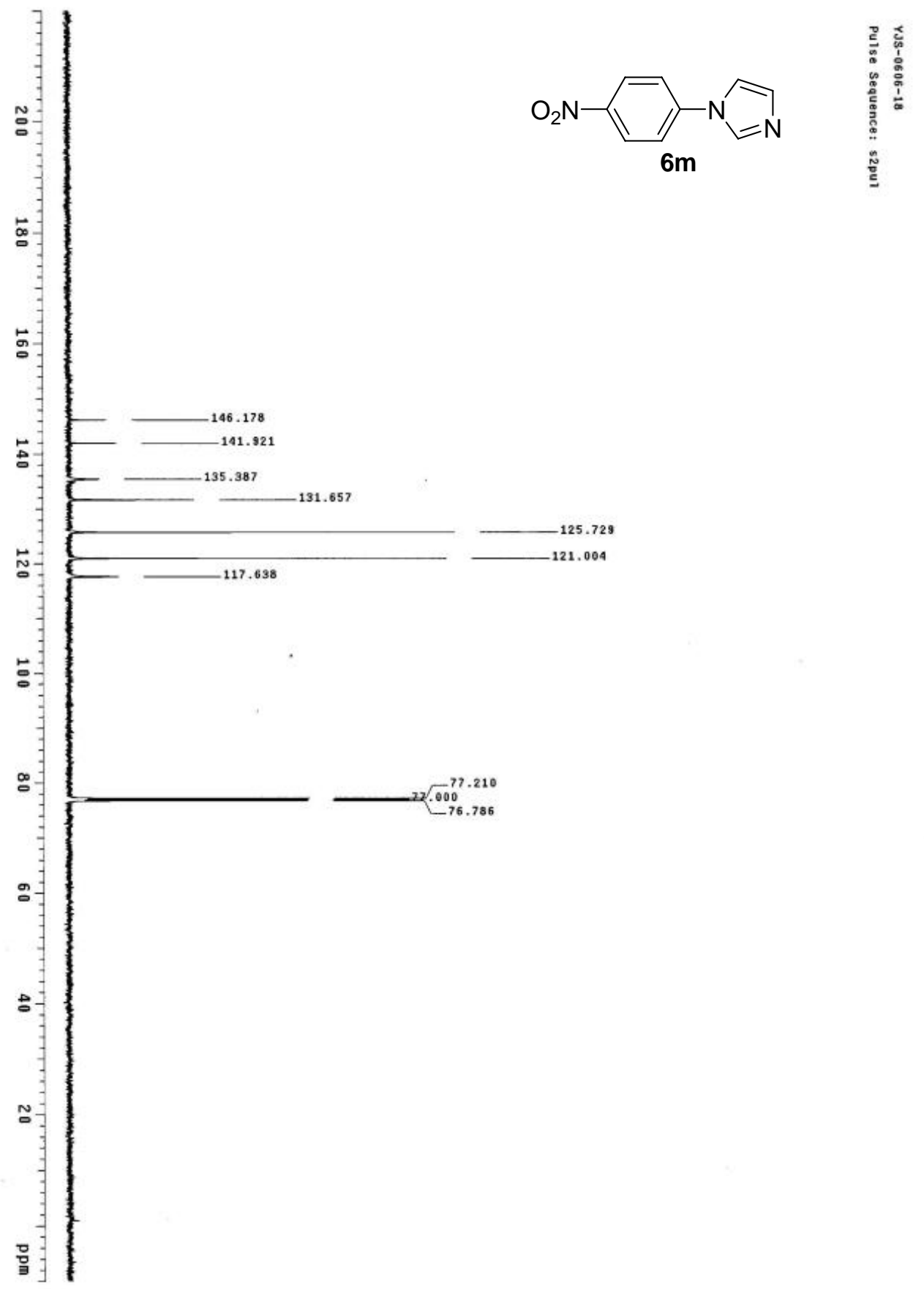




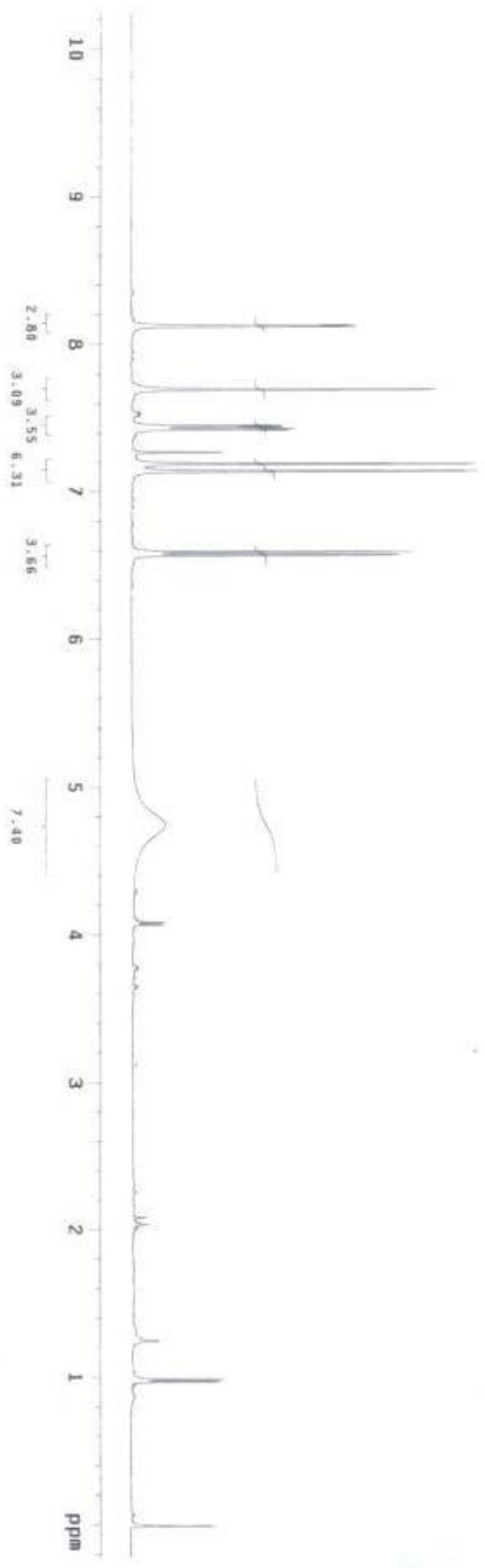

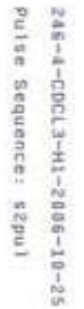
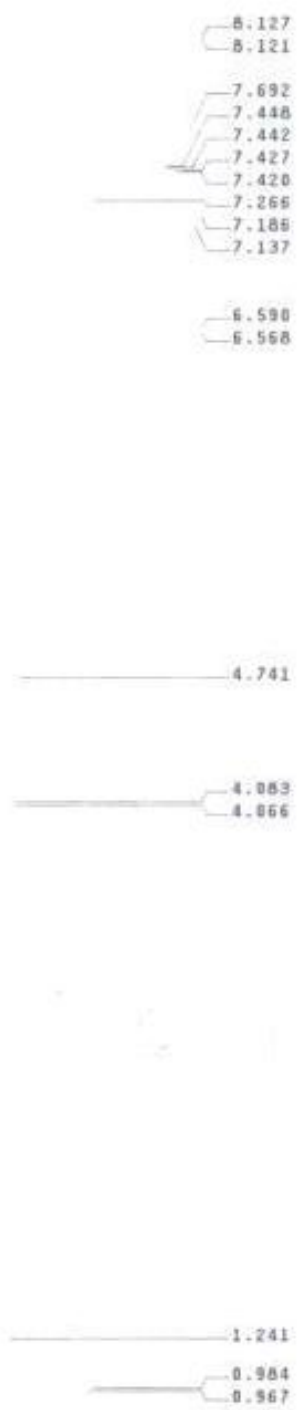

$-0.013$ 

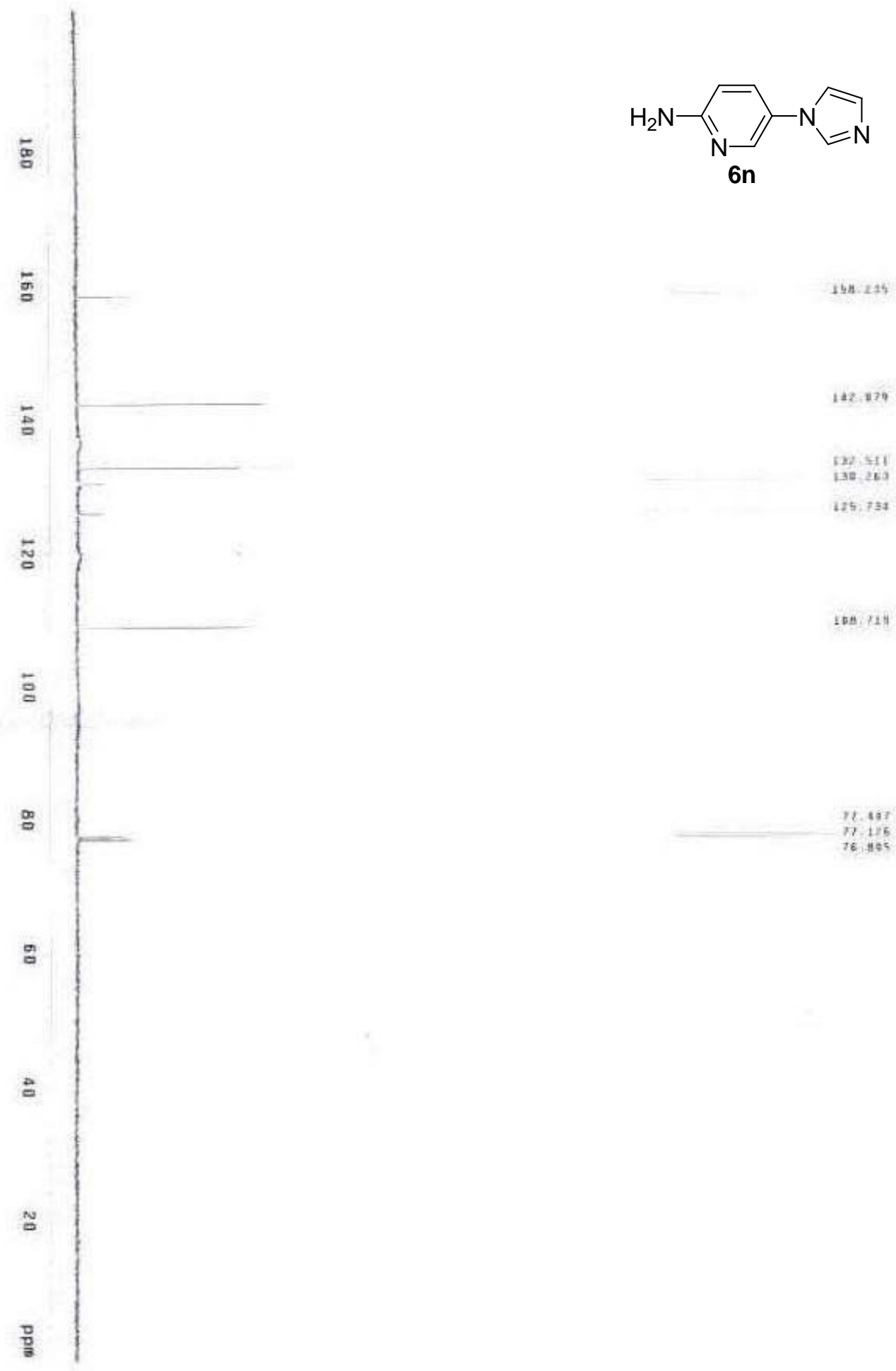


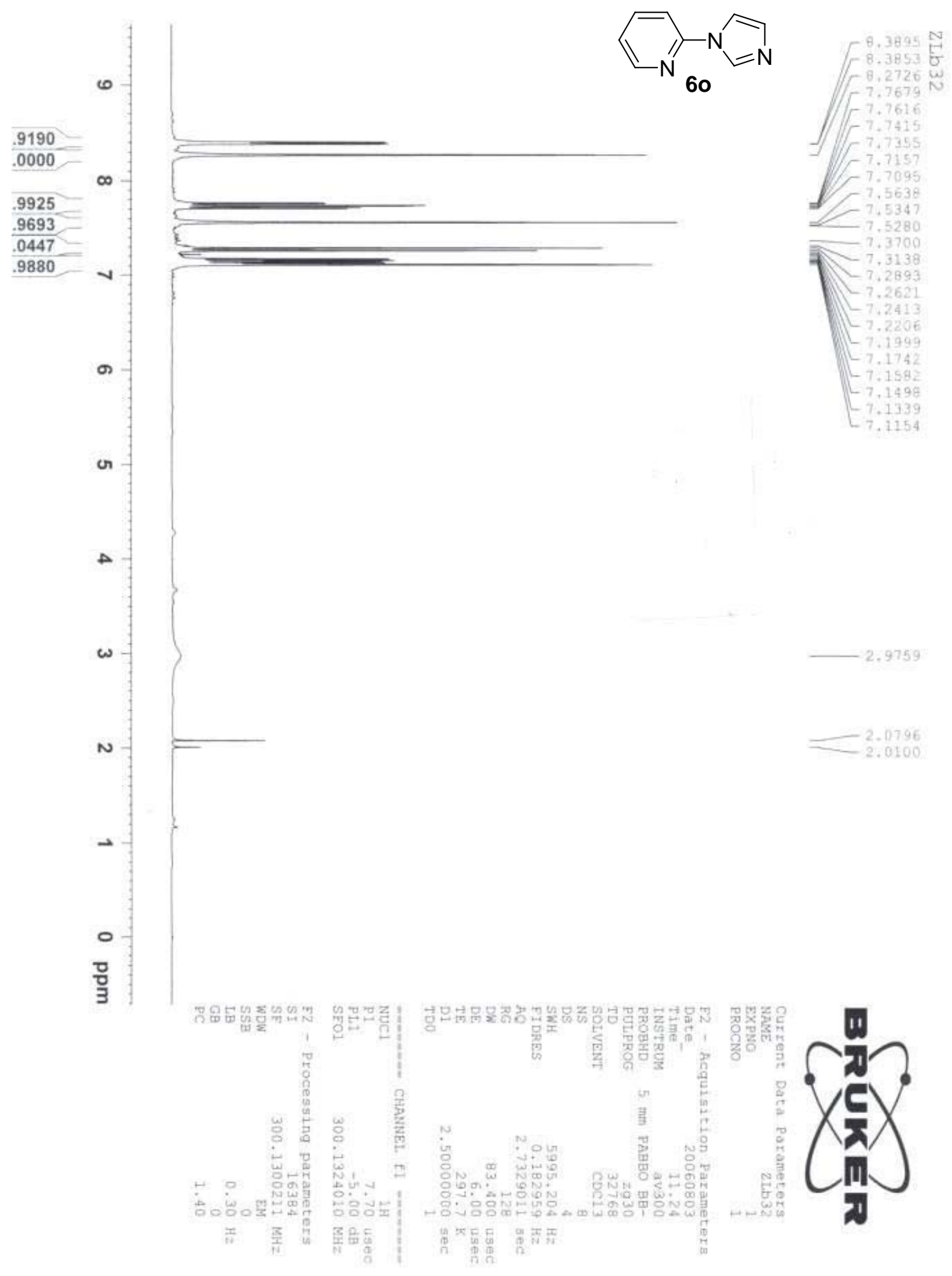




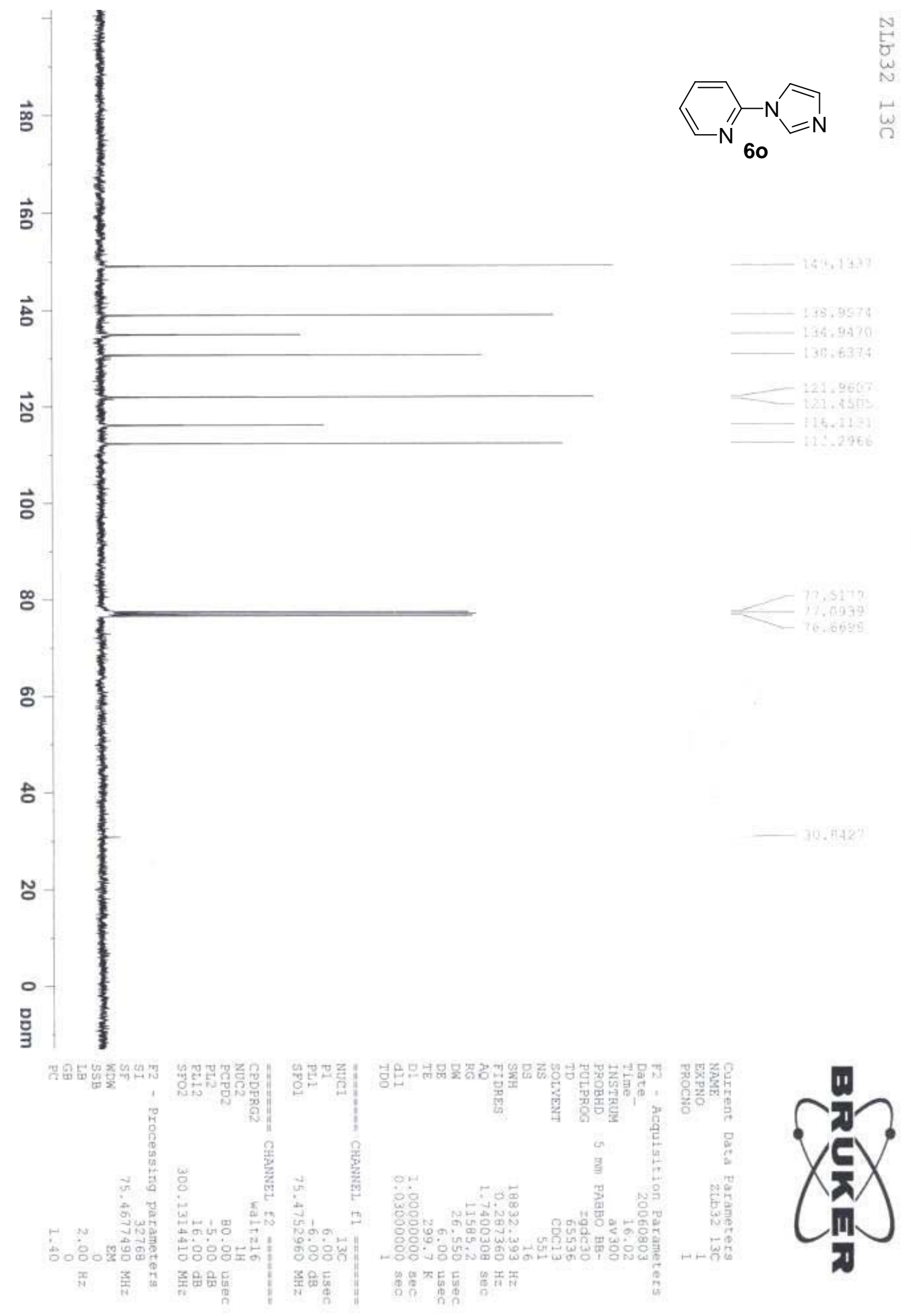




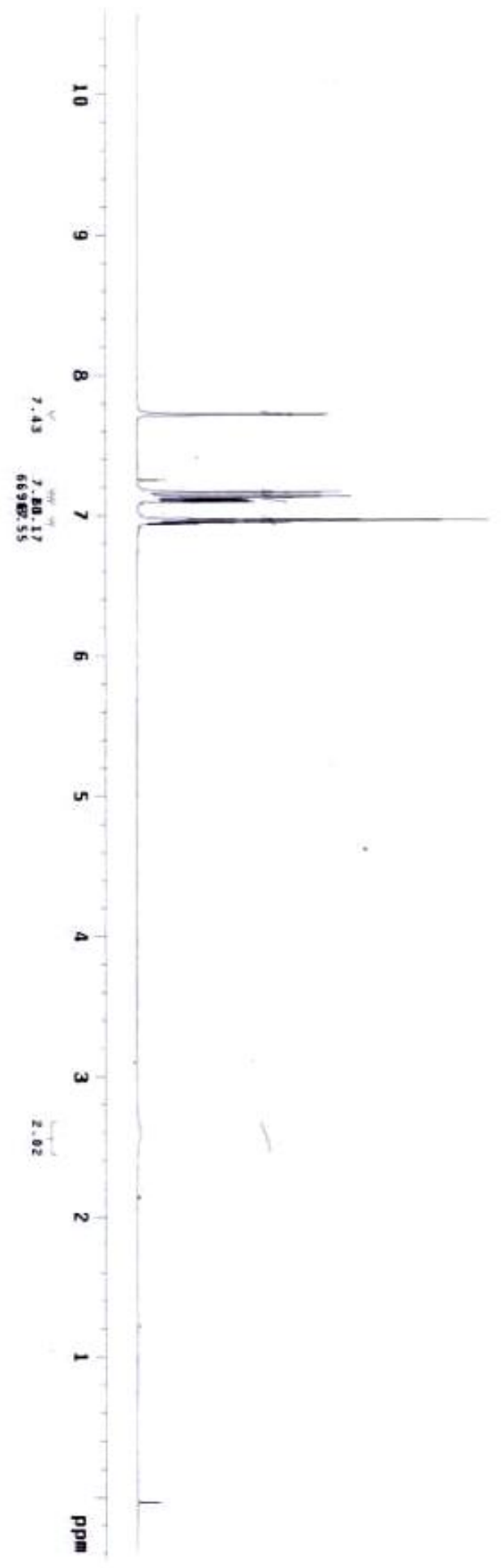

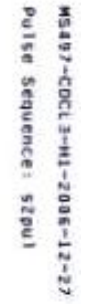
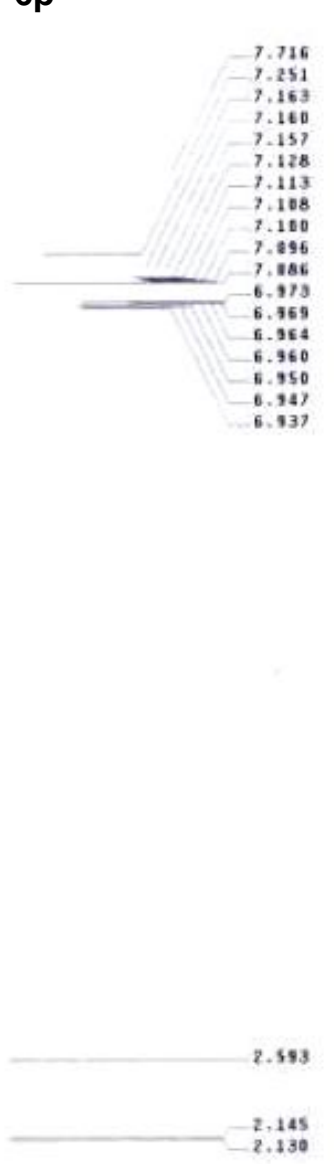


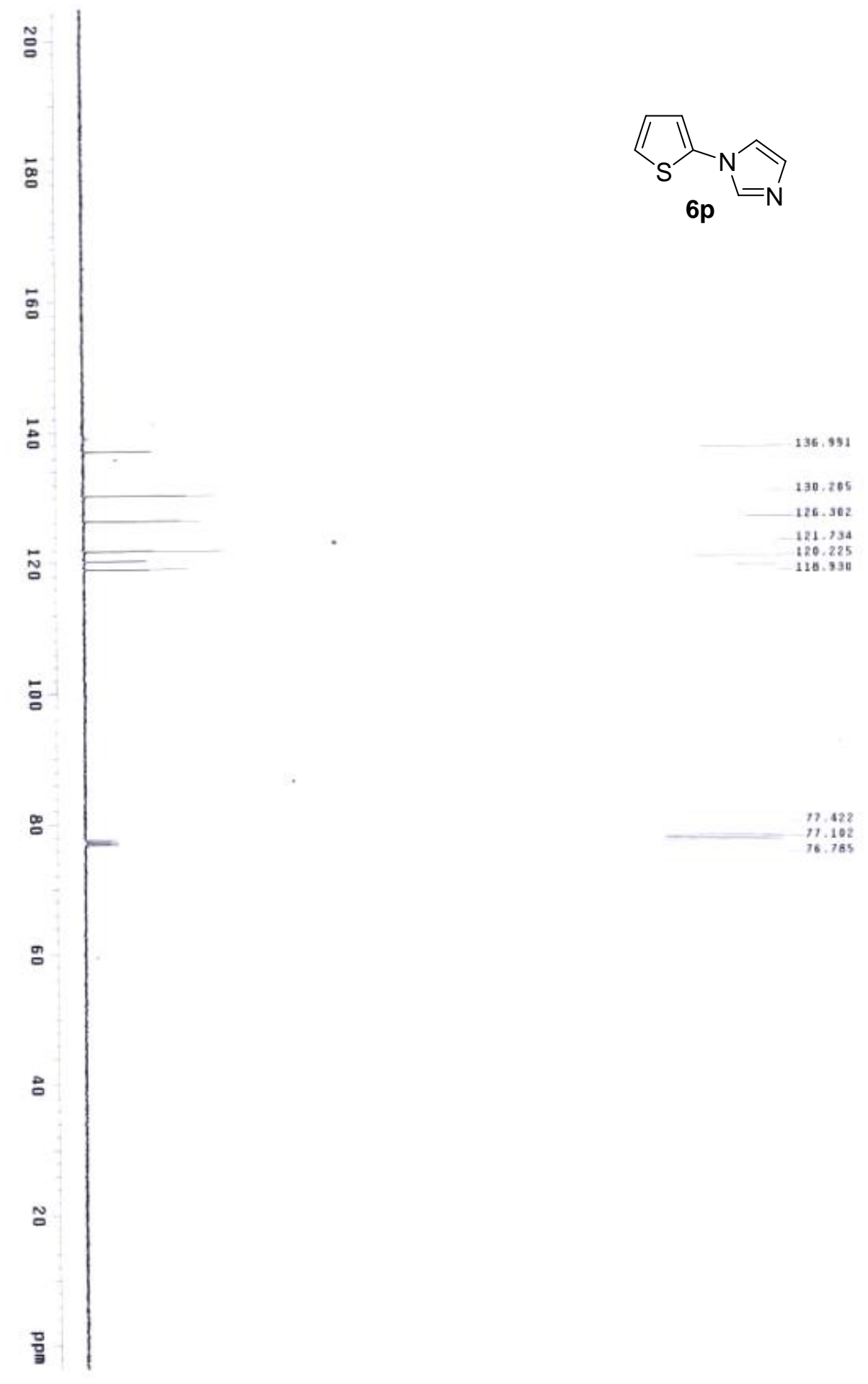

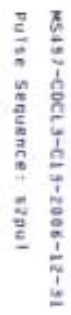

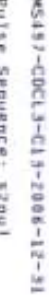



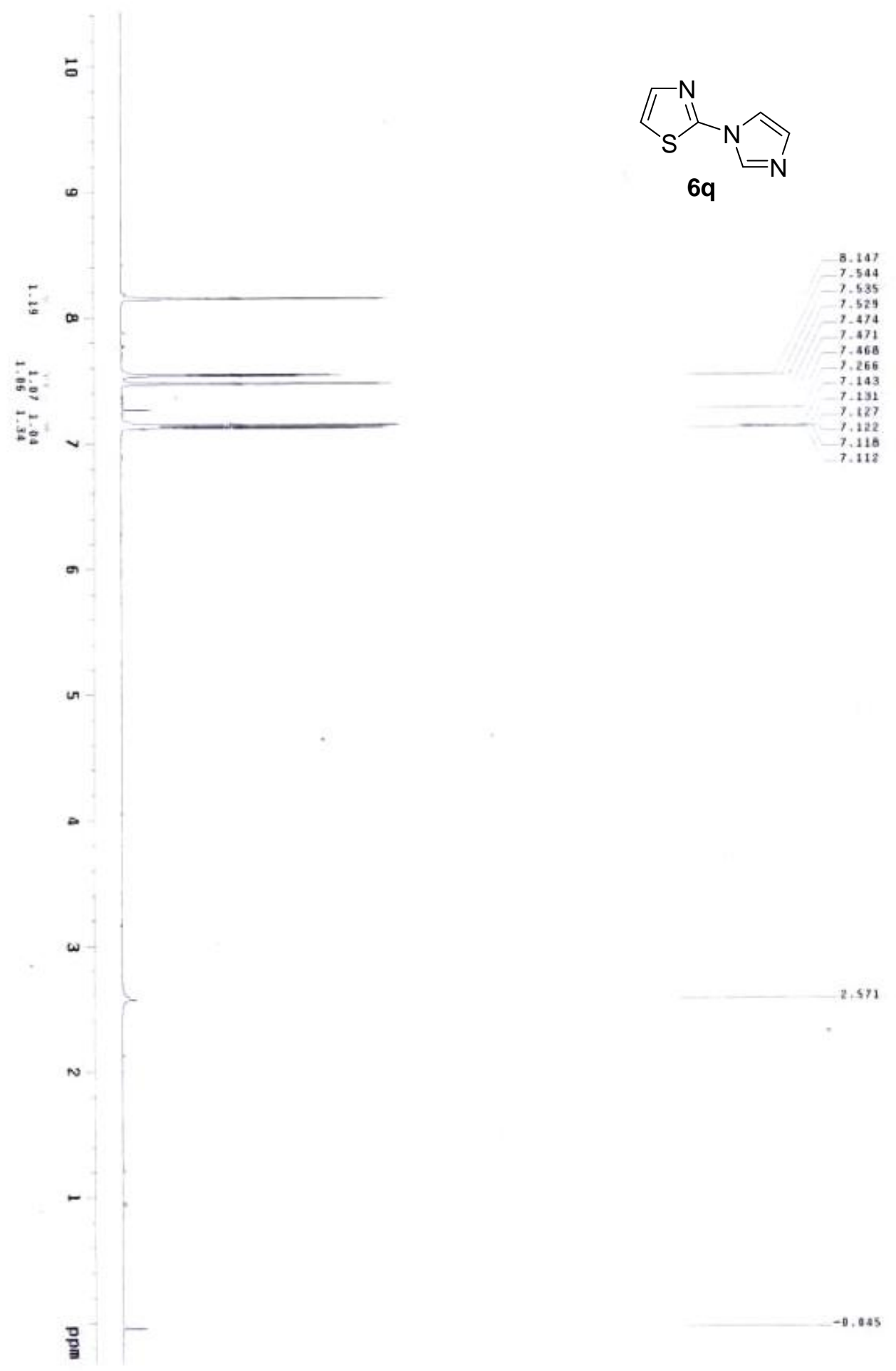

2.571 

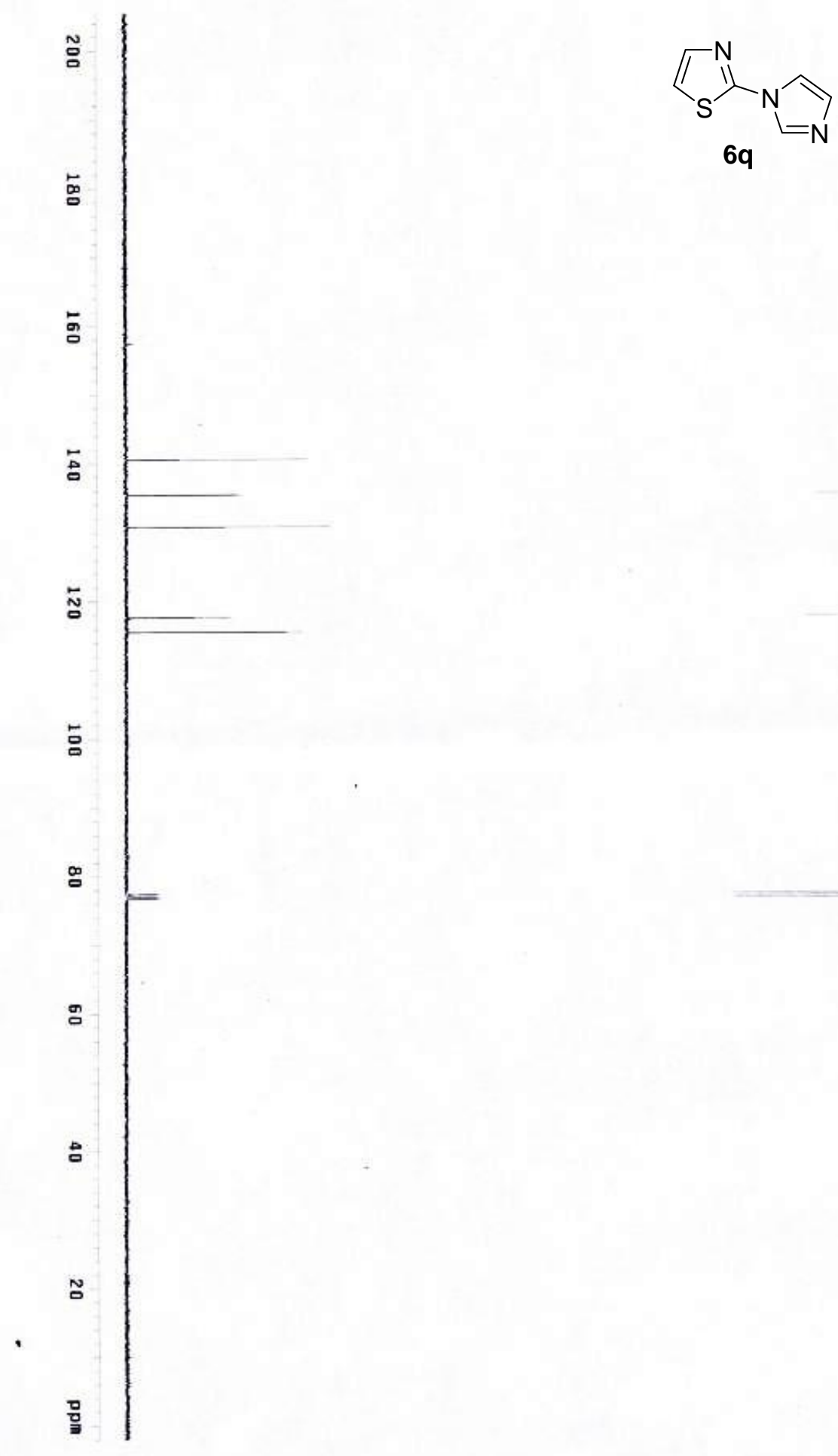

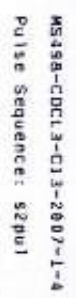

140.523

135.436

130.776

117.625
115.508 


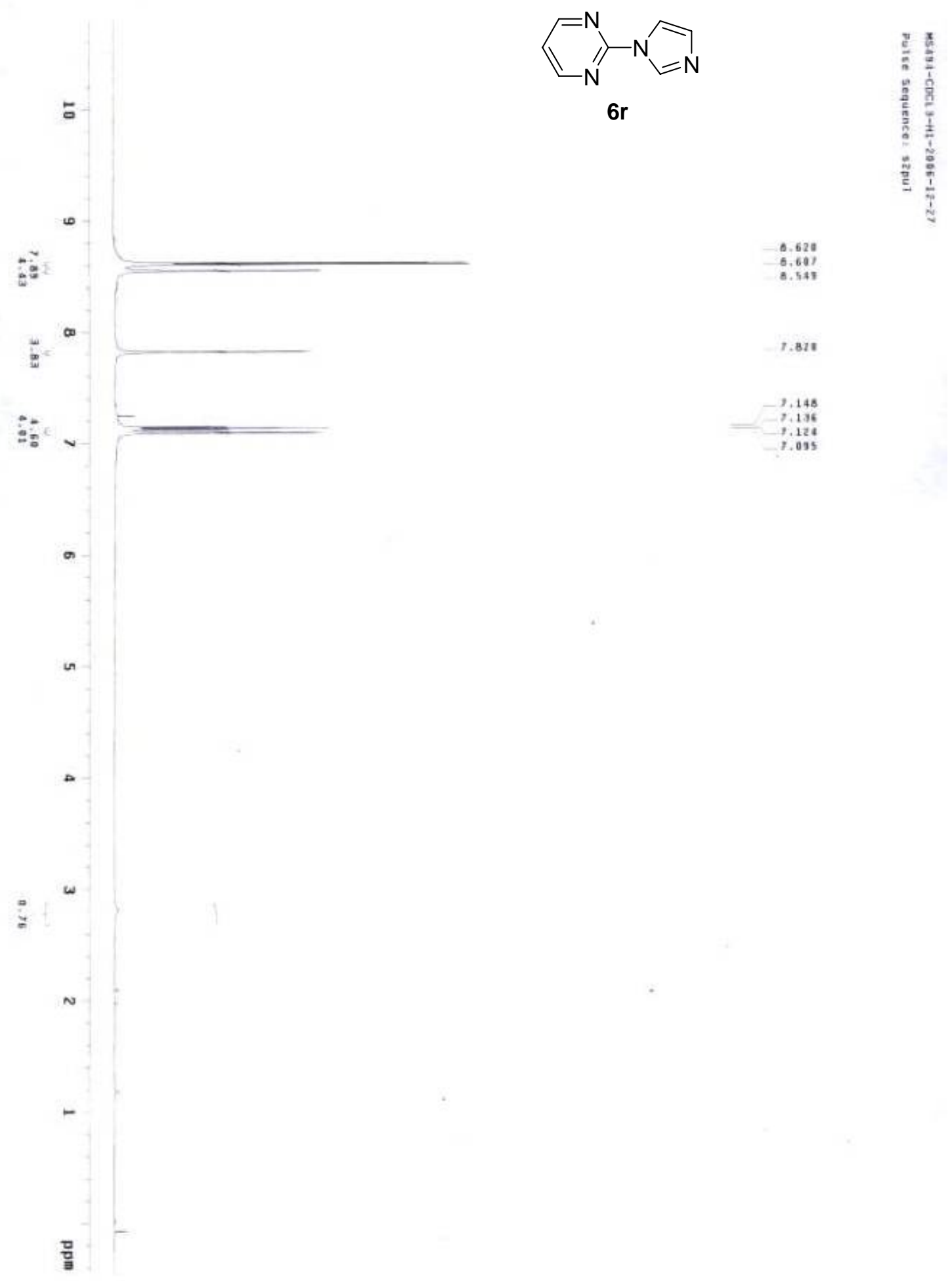



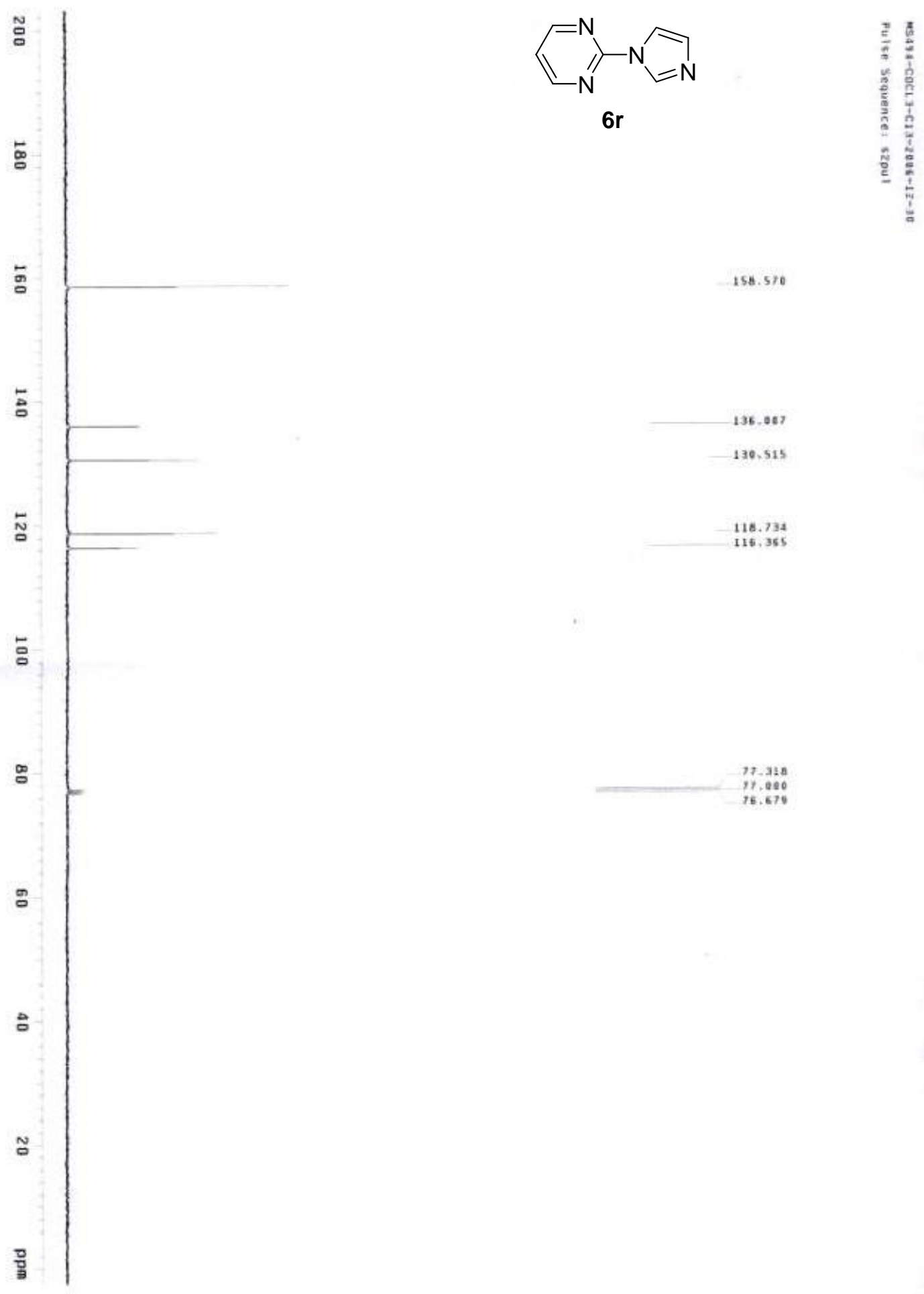

158.570

136.067

130.515

116,355

77. 318

$7 x^{7,000}$

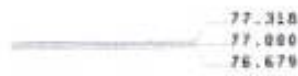




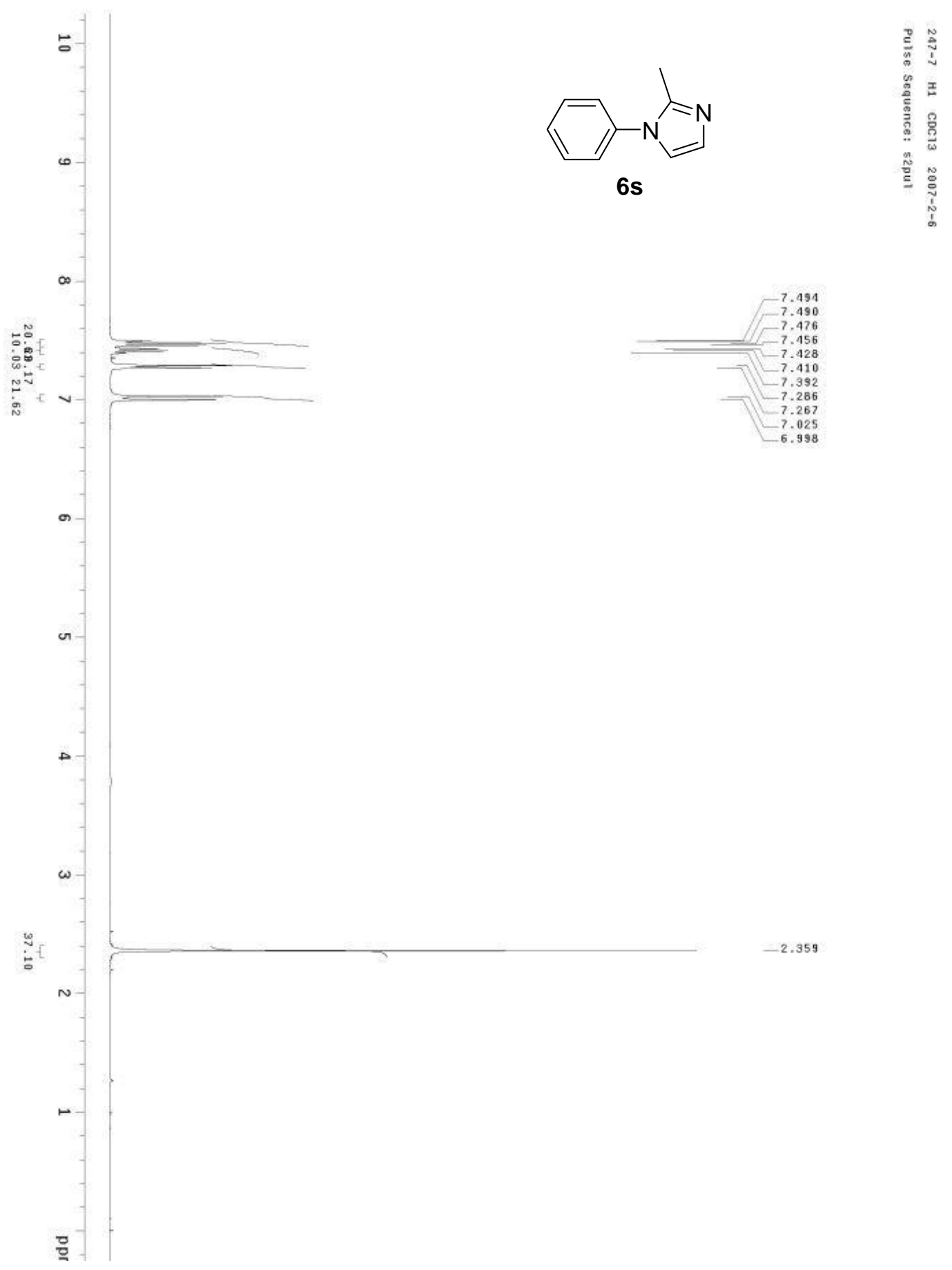




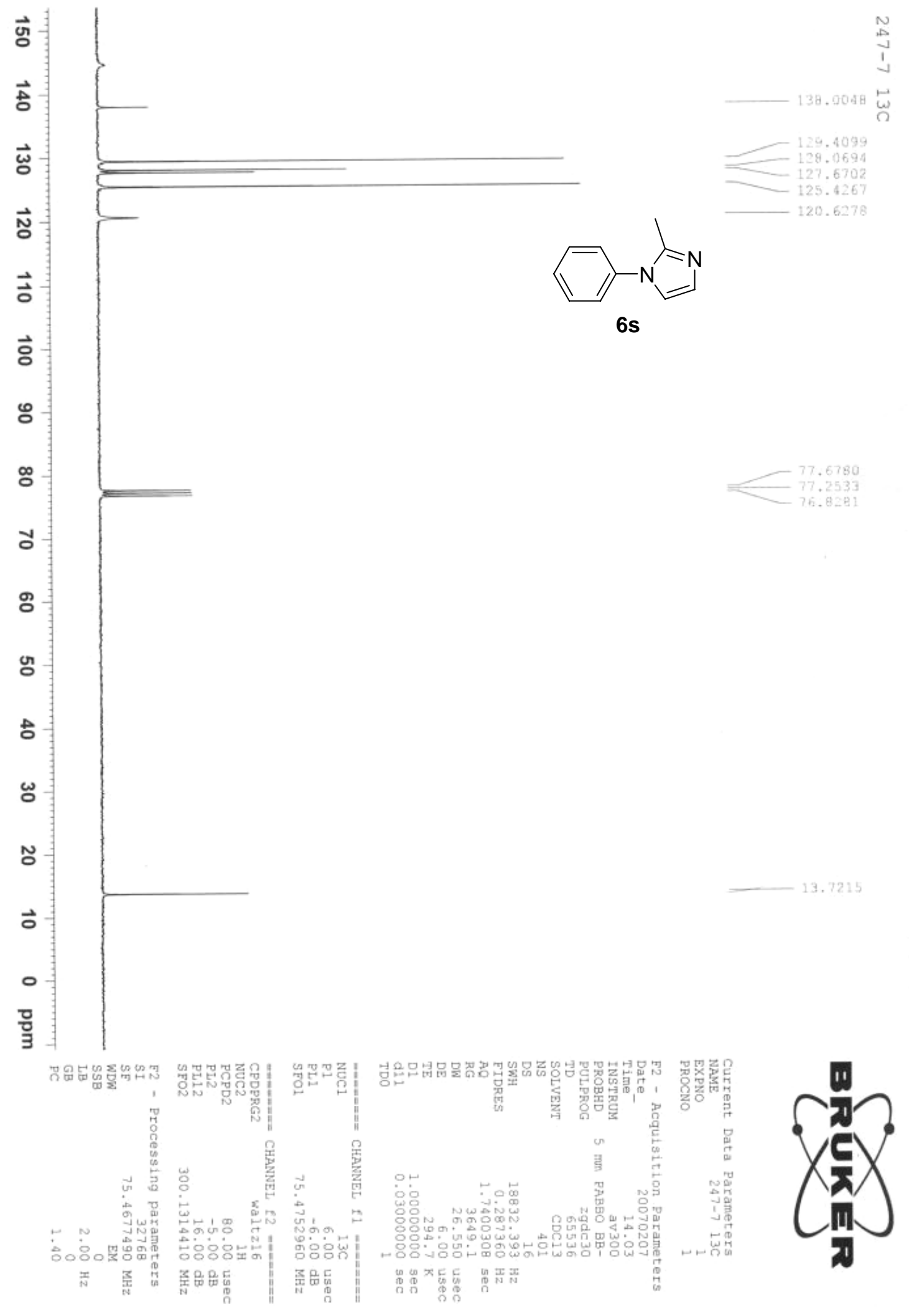




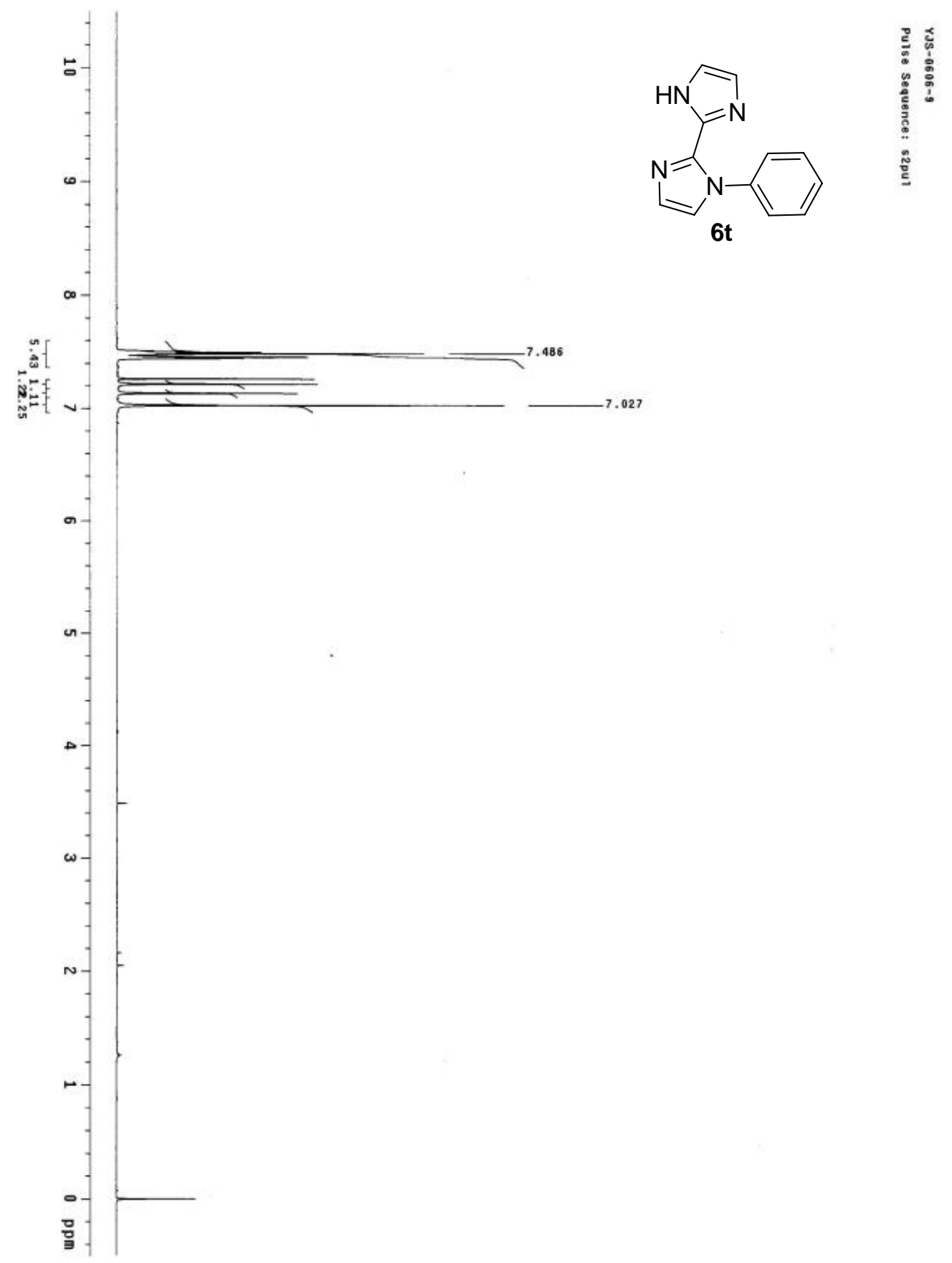



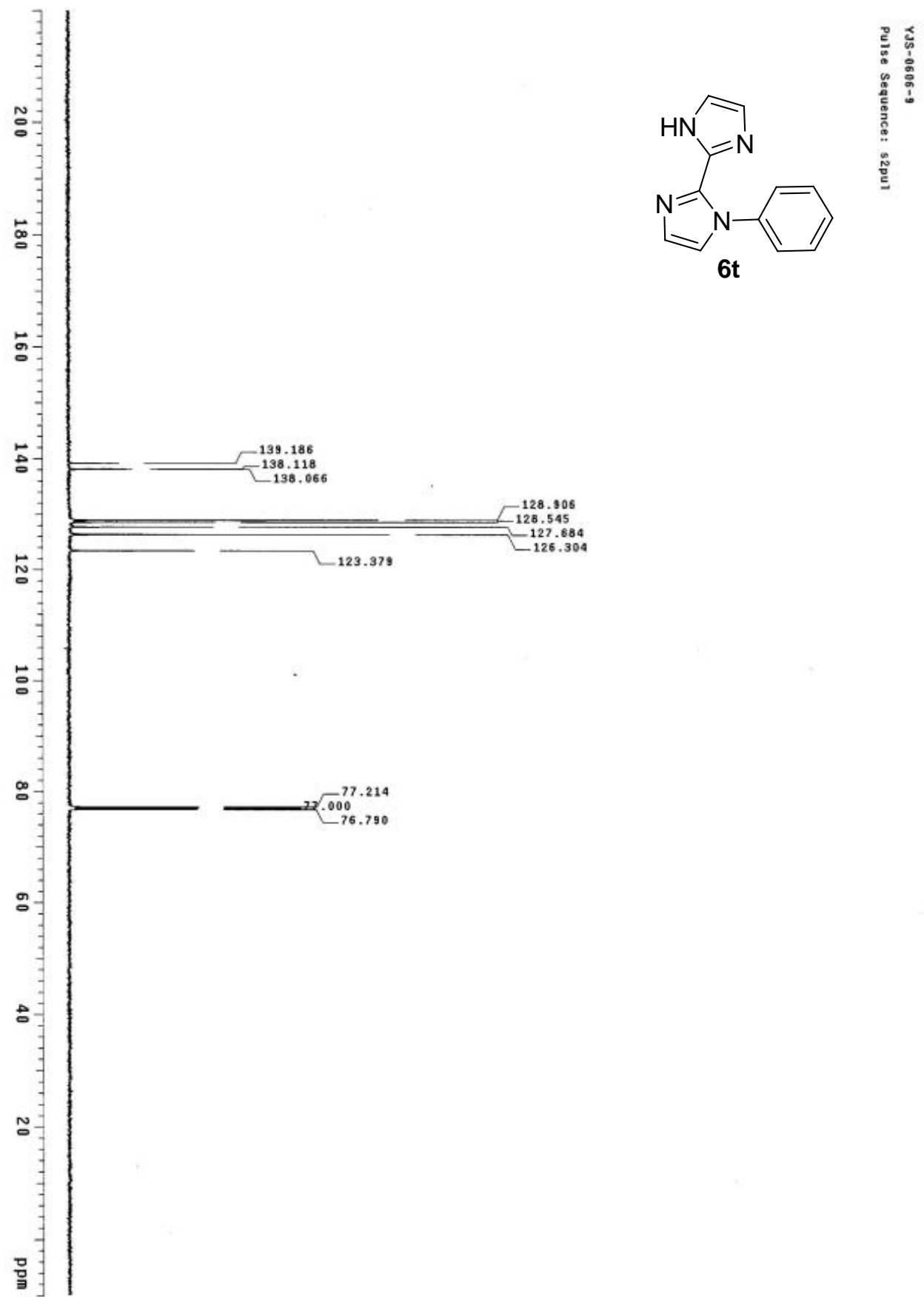


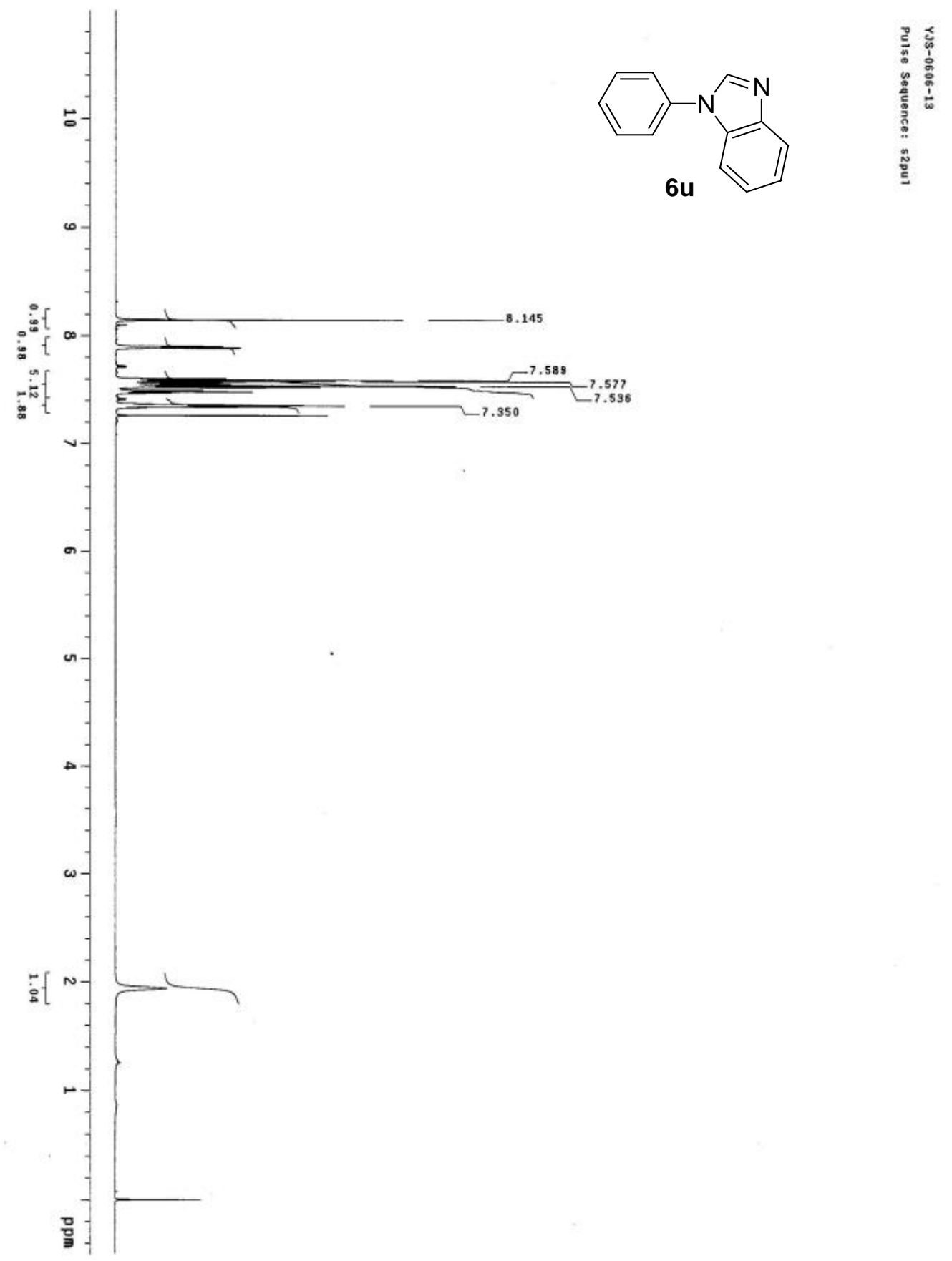




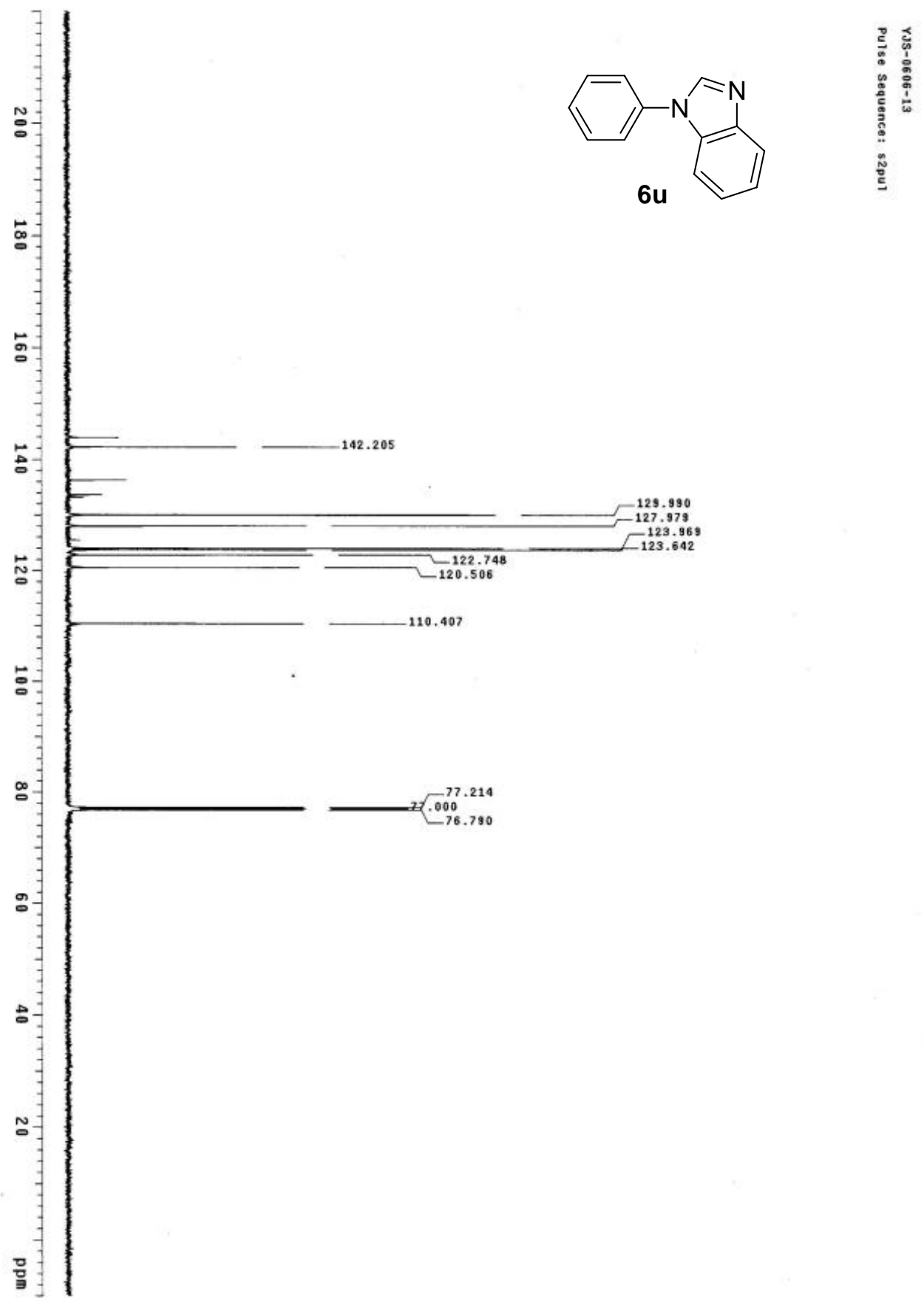




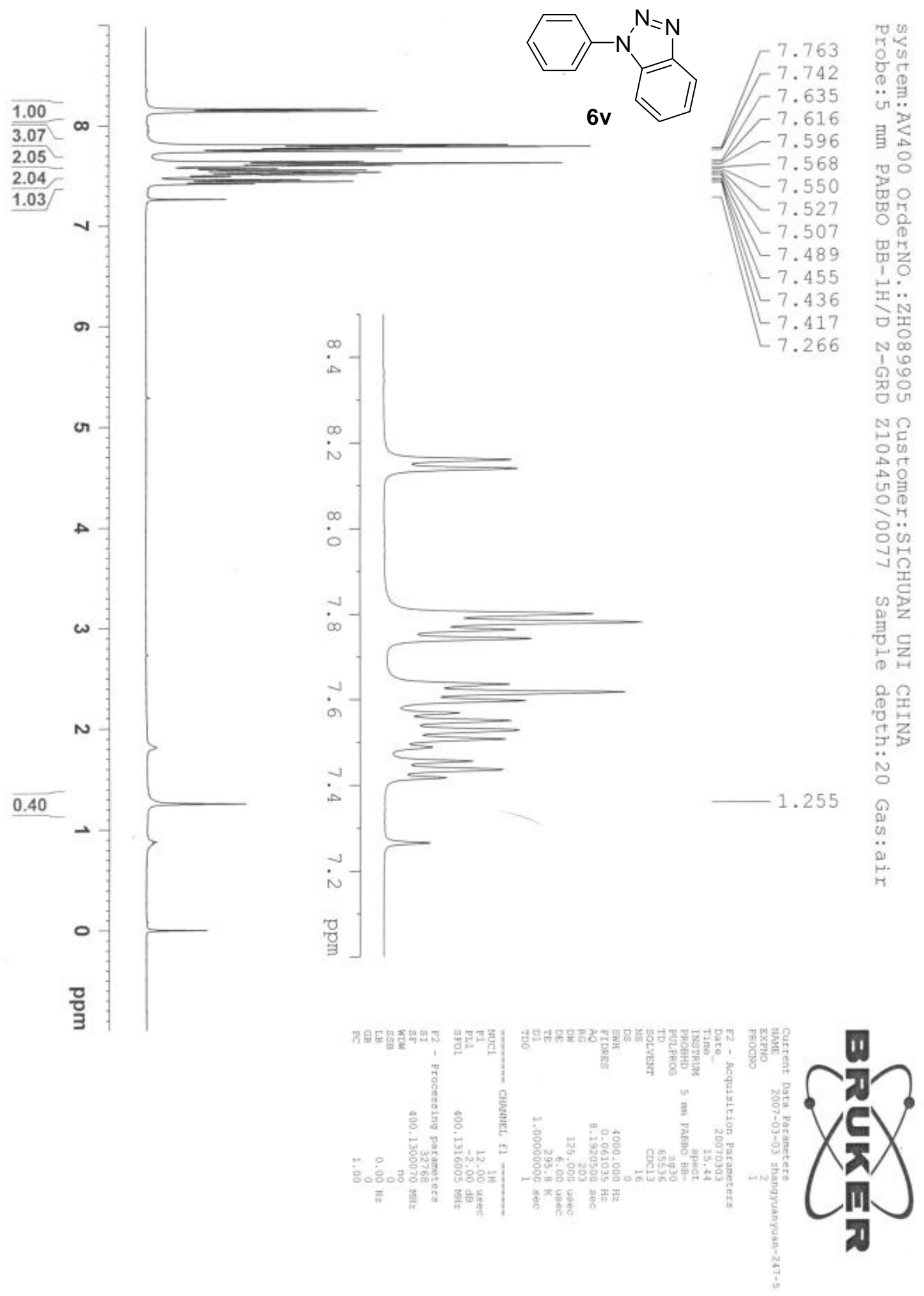




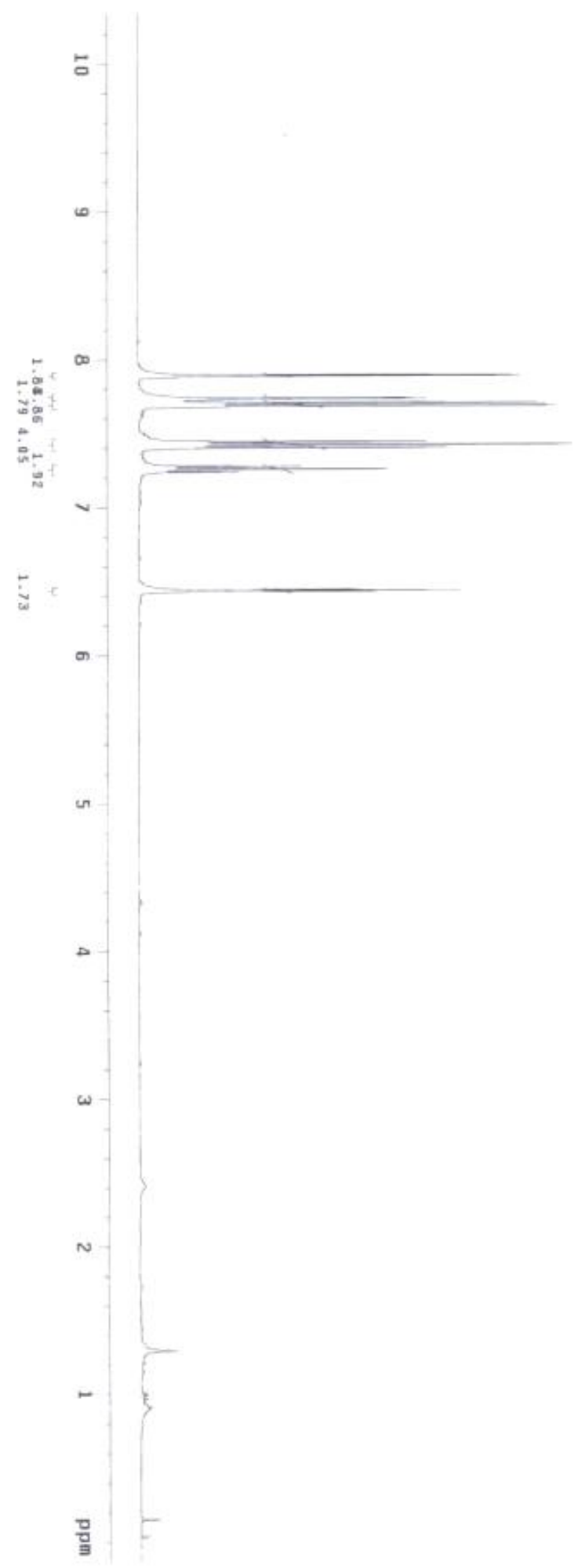

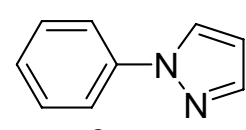

$6 w$

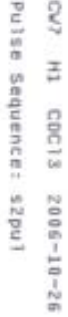

$-1.293$

0.151 


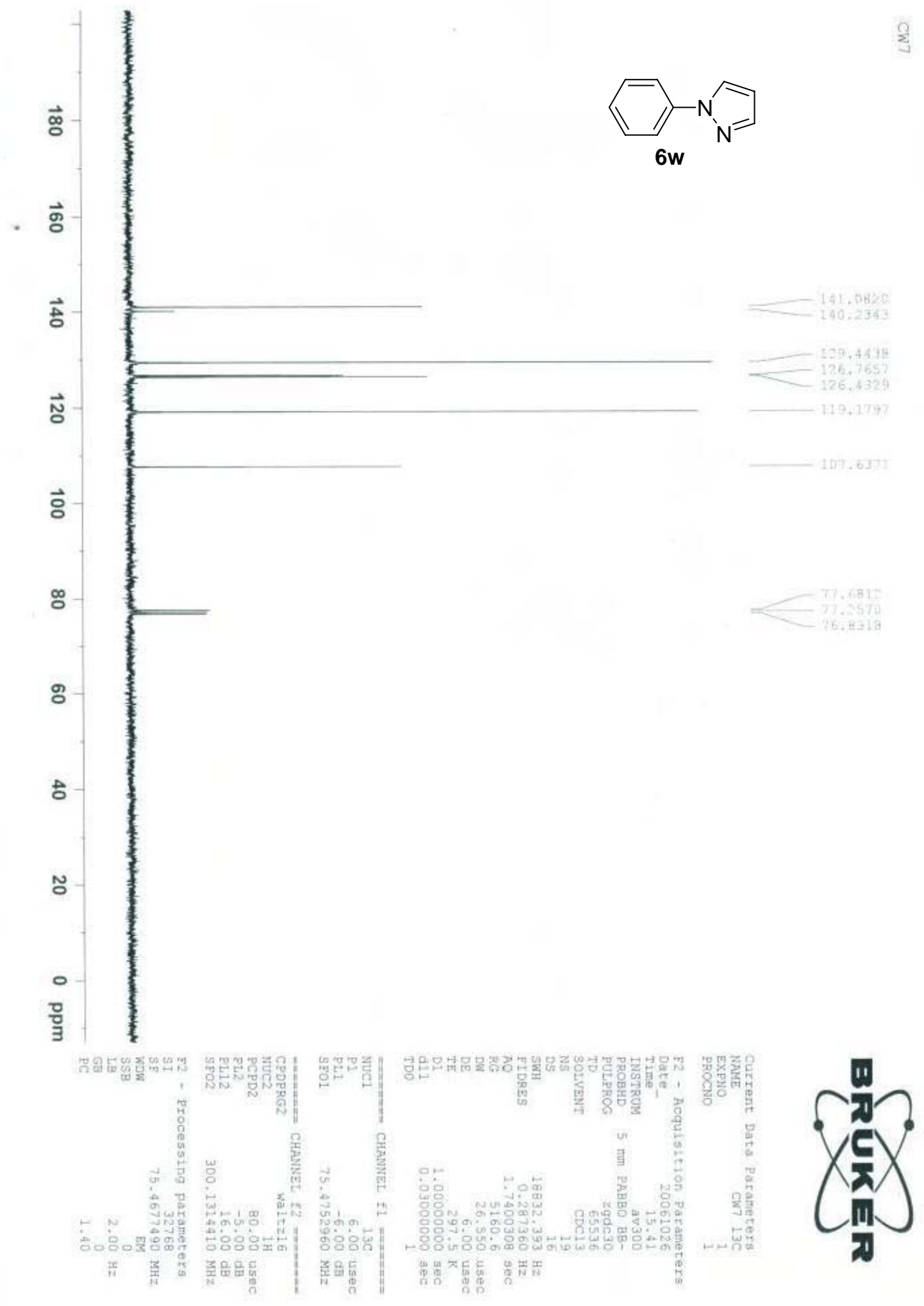




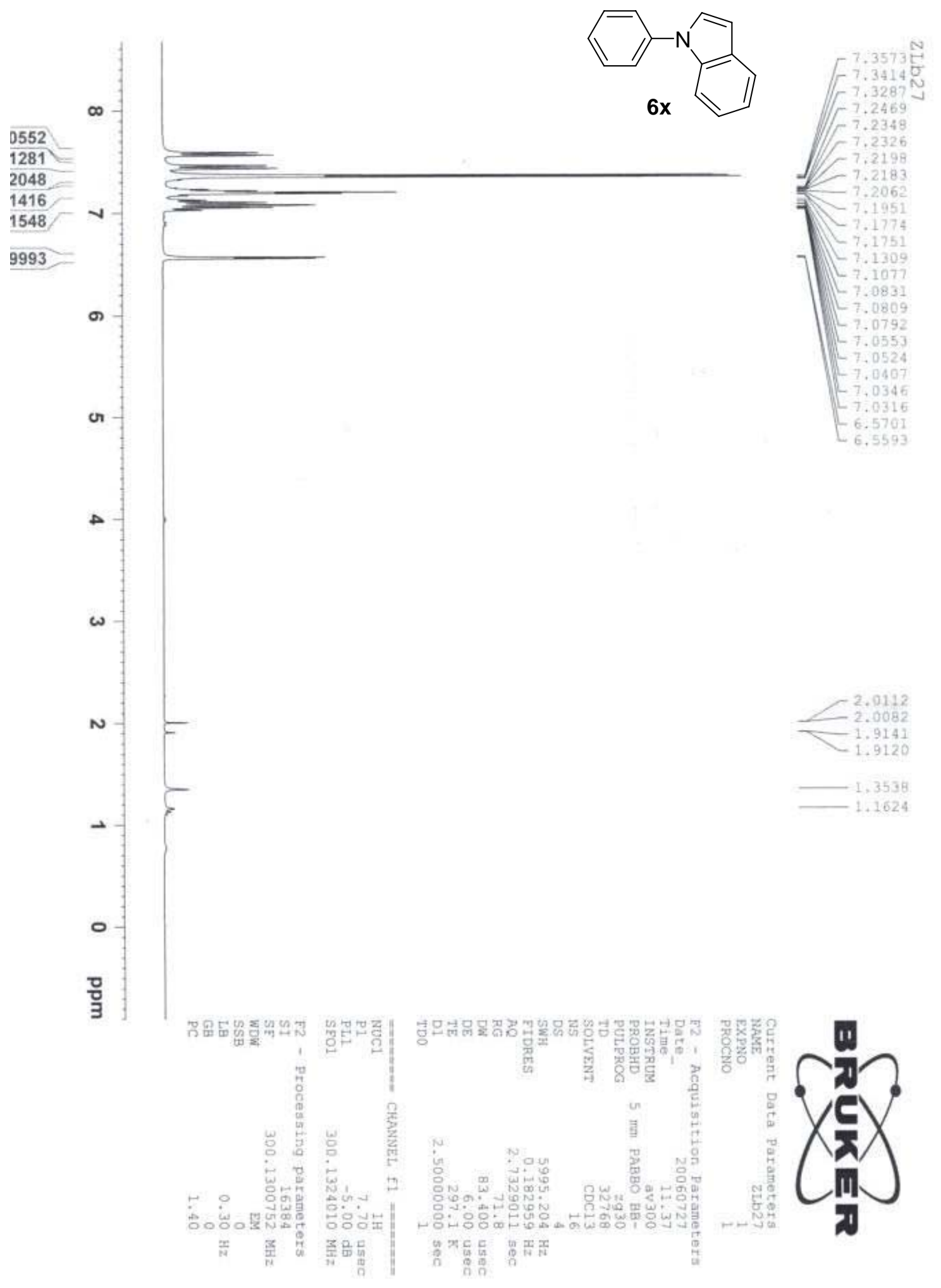




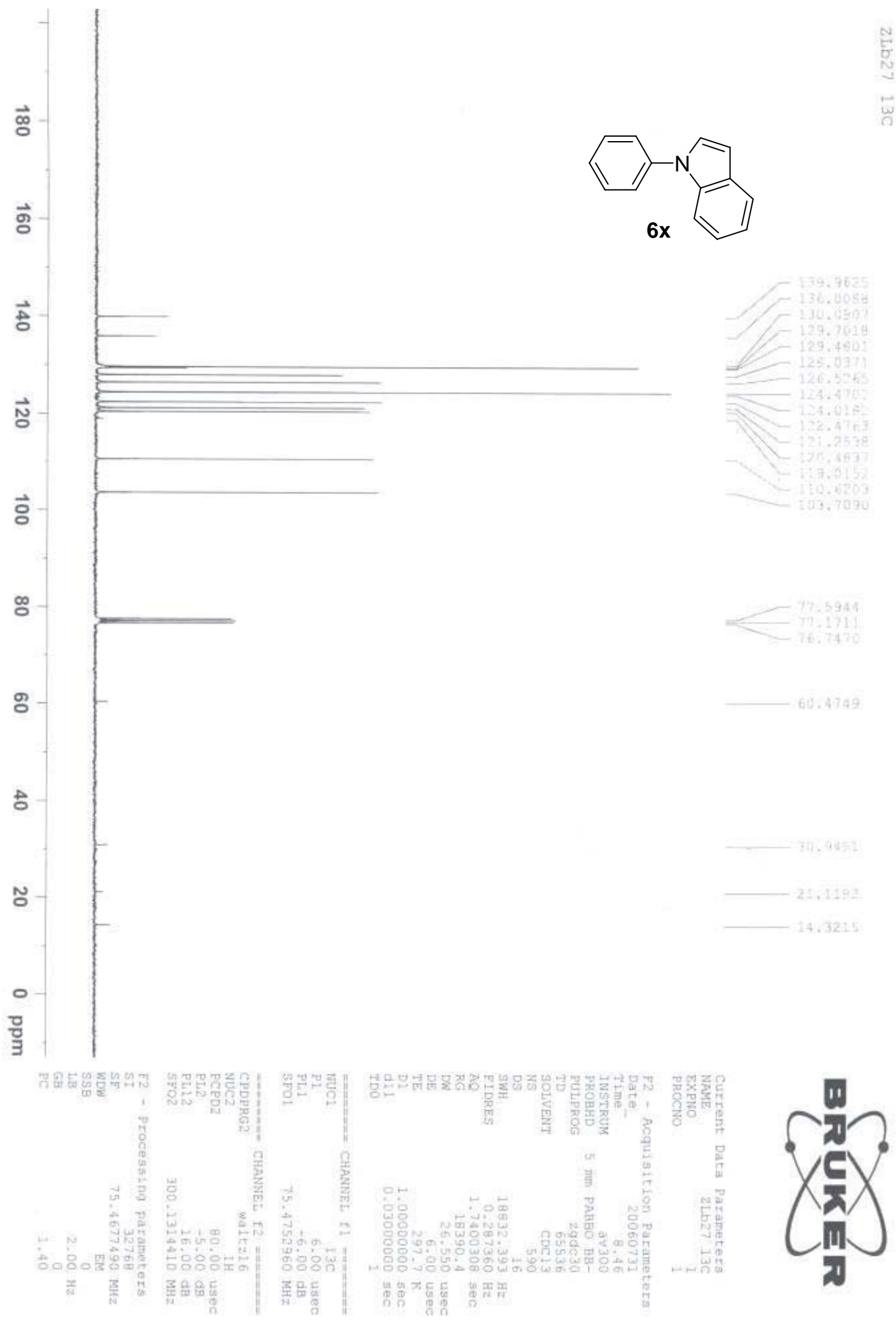




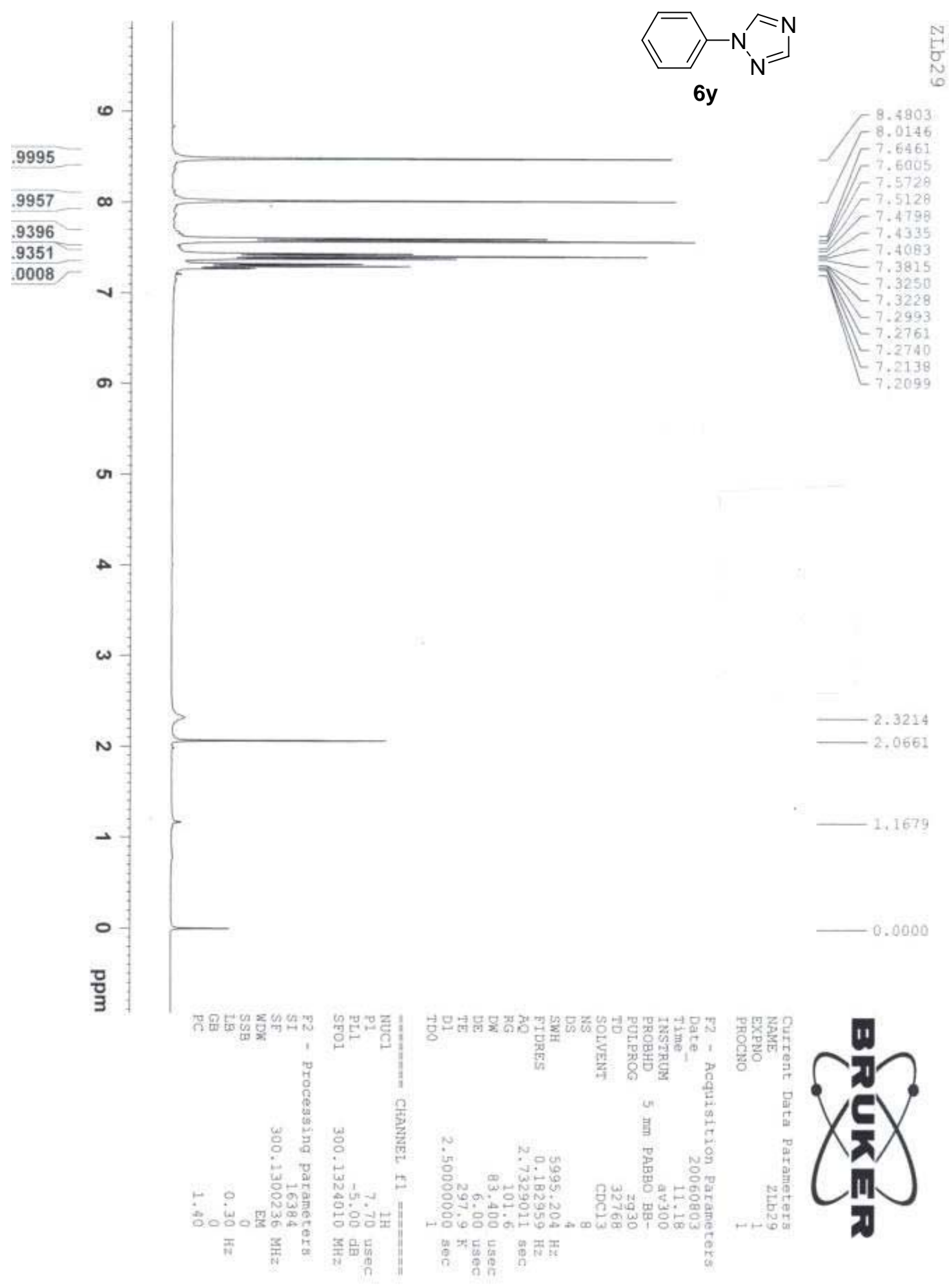




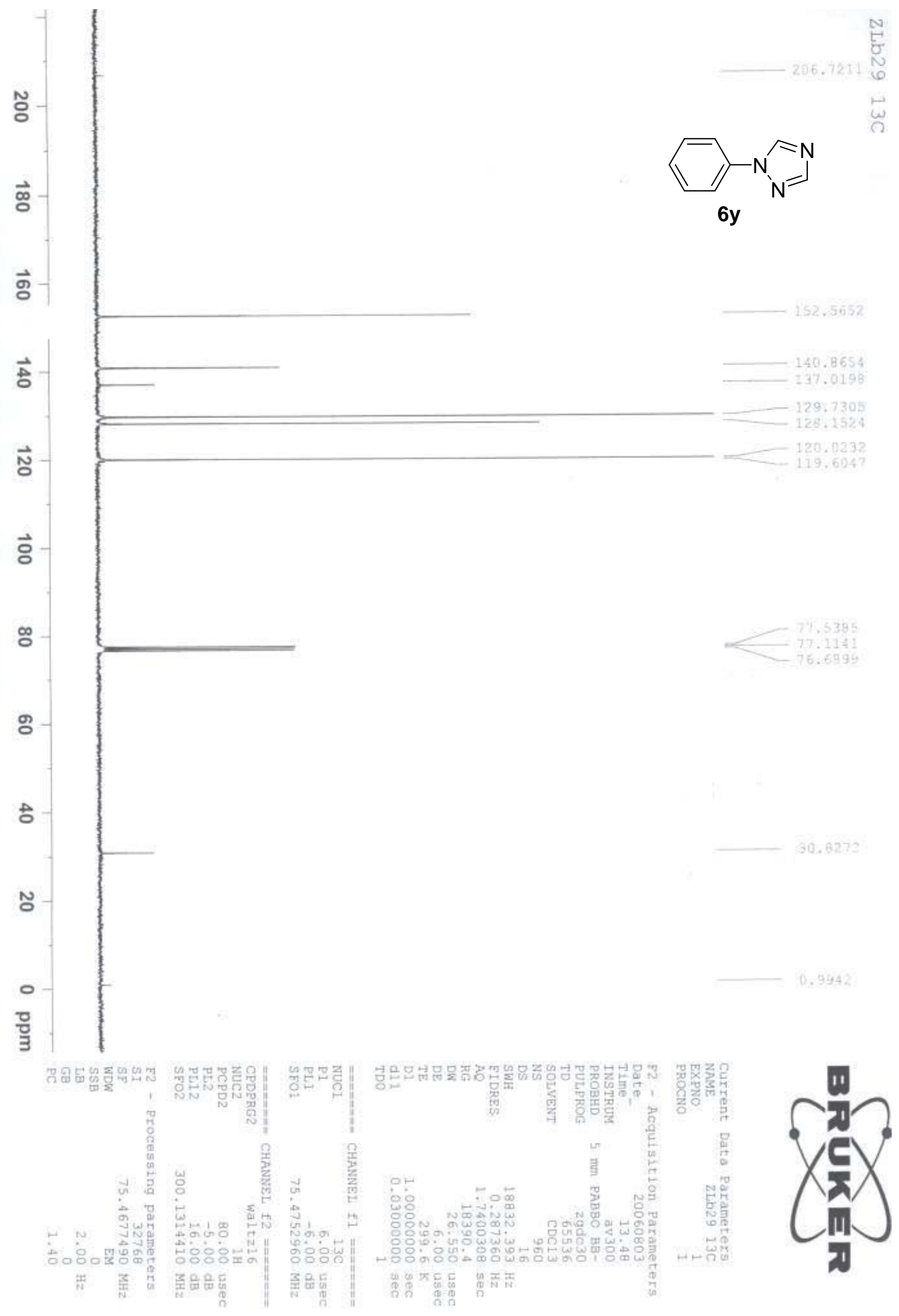

